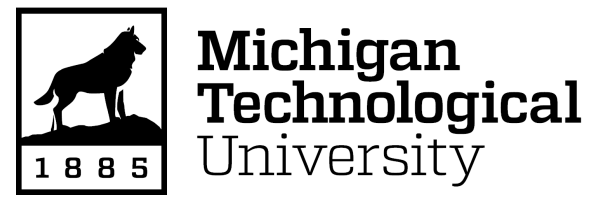

Michigan Technological University Digital Commons @ Michigan Tech

Dissertations, Master's Theses and Master's Reports

2019

\title{
ALGORITHMS AND OPTIMAL CONTROL FOR SPACECRAFT MAGNETIC ATTITUDE MANEUVERS
}

Mohammed Desouky

Michigan Technological University, madesouk@mtu.edu

Copyright 2019 Mohammed Desouky

\section{Recommended Citation}

Desouky, Mohammed, "ALGORITHMS AND OPTIMAL CONTROL FOR SPACECRAFT MAGNETIC ATTITUDE MANEUVERS", Open Access Dissertation, Michigan Technological University, 2019.

https://doi.org/10.37099/mtu.dc.etdr/968

Follow this and additional works at: https://digitalcommons.mtu.edu/etdr

Part of the Navigation, Guidance, Control and Dynamics Commons 


\title{
ALGORITHMS AND OPTIMAL CONTROL FOR SPACECRAFT MAGNETIC ATTITUDE MANEUVERS
}

By

Mohammed Desouky

\author{
A DISSERTATION \\ Submitted in partial fulfillment of the requirements for the degree of \\ DOCTOR OF PHILOSOPHY \\ In Mechanical Engineering-Engineering Mechanics \\ MICHIGAN TECHNOLOGICAL UNIVERSITY \\ 2019 \\ (C) 2019 Mohammed Desouky
}



This dissertation has been approved in partial fulfillment of the requirements for the Degree of DOCTOR OF PHILOSOPHY in Mechanical Engineering-Engineering Mechanics.

\author{
Mechanical Engineering - Engineering Mechanics
}

Dissertation Co-advisor: Dr. Ossama Abdelkhalik

Dissertation Co-advisor: Dr. Bo Chen

Committee Member: Dr. Bong Wie

Committee Member: Dr. Mo Rastgaar

Department Chair: Dr. William Predebon 



\section{Dedication}

To my mother, father, wife and my entire family

who supported me in each step in my life to be who I am.

To my teachers

who didn't hesitate to criticize my work at every stage and provided their academic advice, constant support and encouragement. 



\section{Contents}

List of Figures . . . . . . . . . . . . . . . . . . . . . . xiii

List of Tables . . . . . . . . . . . . . . . . . . . . . . . . . . xxi

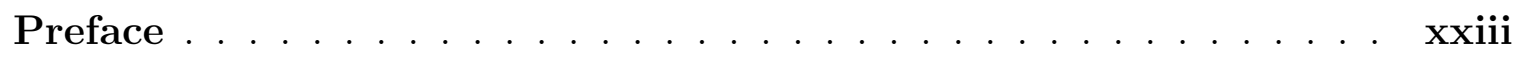

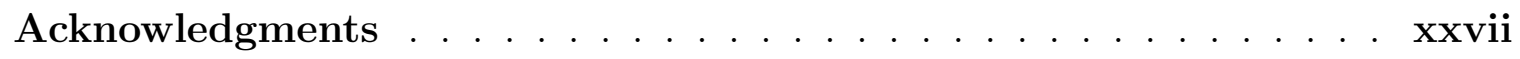

List of Abbreviations . . . . . . . . . . . . . . . . . . xxix

Nomenclature ...................... . . . . . . . . . . . . . . .

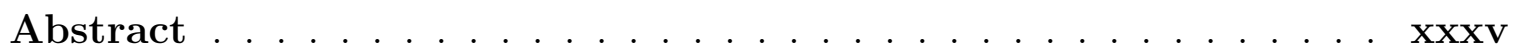

1 Introduction . . . . . . . . . . . . . . . . . . . . . 1

1.1 Background . . . . . . . . . . . . . . . . . . 2

1.2 Magnetic Attitude Control Challenges . . . . . . . . . . . . . 5

1.2.1 Magnetic Attitude Control Singularity . . . . . . . . . . 5

1.2 .2 Magnetometer errors . . . . . . . . . . . . . . 7

1.2.2.1 Manufacturing Tolerance Errors . . . . . . . . . . 7 
1.2.2.2 Surrounding Environment Errors . . . . . . . . . 8

1.2 .3 Magnetometer Calibration . . . . . . . . . . . . . . 12

1.3 Magnetic Attitude Control Maneuvers . . . . . . . . . . . . . . . . 13

1.3 .1 Detumbling Maneuvers . . . . . . . . . . . . . . . . . . . . . 13

1.3 .2 Three-Axis Attitude Control Maneuvers . . . . . . . . . . . 17

1.4 Contributions of This Dissertation . . . . . . . . . . . . . . . . . . 21

1.5 Outline of This Dissertation . . . . . . . . . . . . . . . . . . . . . . 22

2 Spacecraft and environment models . . . . . . . . . . . . . . 25

2.1 Coordinate reference frames . . . . . . . . . . . . . . 26

2.2 Notation . . . . . . . . . . . . . . . . . . . . . . . . 27

2.3 Spacecraft rotational kinetic and kinematic models . . . . . . . . . 28

$2.3 .1 \quad$ Spacecraft kinematics model . . . . . . . . . . . . . . . . . 28

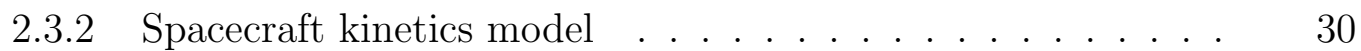

2.4 Space environment models . . . . . . . . . . . . . . . . . . . . 32

$2.4 .1 \quad$ Orbit propagator . . . . . . . . . . . . . . . 32

2.4 .2 Geomagnetic field model . . . . . . . . . . . . . . . . . . . . 32

$2.4 .3 \quad$ Gravity Gradient Torque . . . . . . . . . . . . . . . . . . . . 33

2.4 .4 Aerodynamics Torque . . . . . . . . . . . . . . . . . 33

2.4 .5 Solar Radiation Torque . . . . . . . . . . . . . . . . . . . . . 34

2.4 .6 Residual torque . . . . . . . . . . . . . . . . . . 34

2.5 CASSIOPE Spacecraft and Mission . . . . . . . . . . . . . . . . 35 
3 A New Efficient Variant of The B-dot Control for Spacecraft Magnetic Detumbling . . . . . . . . . . . . . . . . . . . . . . . 37

3.1 Magnetic Detumbling Control Law . . . . . . . . . . . . . . 38

3.1 .1 The Modified B-dot Control Law . . . . . . . . . . . . . . . 39

3.1 .2 Lyapunov Analysis . . . . . . . . . . . . . . . . . . . . . 43

3.1.3 The Proposed B-dot Law from Geometric Point of View . . 49

$3.2 \quad$ Magnetometer Errors and Frequency Aliasing Considerations . . . . $\quad 52$

3.3 Simulations and Discussions . . . . . . . . . . . . . . . . . 55

3.4 Conclusion . . . . . . . . . . . . . . . . . 66

4 Time-Optimal Magnetic Attitude Detumbling . . . . . . . . . . . 69

4.1 Time-Optimal Control . . . . . . . . . . . . . . . . 70

$4.1 .1 \quad$ Case 1: Using Angular Velocity Measurements . . . . . . . 71

4.1.2 Case 2: Using Only Ambient Magnetic Field Measurements 80

4.2 Simulation Results with Discussion . . . . . . . . . . . . 87

4.3 Conclusion . . . . . . . . . . . . . . . . . . . . . . . . . . . . 97

5 Improved Spacecraft Magnetic Attitude Maneuvering . . . . . . 99

5.1 Magnetic Rods Duty Cycle Extension . . . . . . . . . . . . . . . . . 100

5.2 Magnetic Field Pseudo Measurement . . . . . . . . . . . . . . . . . 103

5.2 .1 Torque Computation . . . . . . . . . . . . . . 103

5.2 .2 Regularization of The Singular System . . . . . . . . . . . . 104

5.3 Control Law . . . . . . . . . . . . . . . . . . . . . . . . . . 109 
$5.3 .1 \quad$ Stability Analysis $\ldots \ldots \ldots \ldots$

5.3 .2 Gain selection Criteria Discussion . . . . . . . . . . . 116

5.4 Numerical Simulation Results $\ldots \ldots \ldots \ldots$

$5.4 .1 \quad$ Inertial Pointing Three-Axis Attitude Control $\ldots \ldots \ldots \ldots$

5.4 .2 Proposed Control Scheme for Extending the Duty Cycle . . 122

5.5 Verification of Magnetic Field Pseudo Measurement Computation Us-

ing Real Data . . . . . . . . . . . . . . . . . . . . . . . . 132

5.6 Conclusion . . . . . . . . . . . . . . . . . . . . . . . 141

6 A Spacecraft Attitude Estimation Algorithm for Efficient Mag-

netic Attitude Maneuvers $\ldots \ldots \ldots$

$6.1 \quad$ Attitude Determination and Control System . . . . . . . . . . 145

$6.1 .1 \quad$ Magnetic Field Pseudo Measurement . . . . . . . . . . . . 147

6.1 .2 Attitude estimation . . . . . . . . . . . . . . . . 153

6.2 Numerical simulations . . . . . . . . . . . . . . . . . . . . 159

$6.3 \quad$ Verification of magnetic field Pseudo Measurement Computation using real data $\ldots \ldots \ldots \ldots \ldots \ldots \ldots \ldots \ldots \ldots$

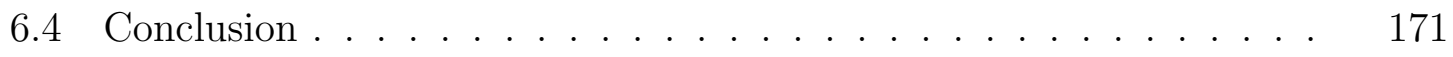

7 Conclusion and Future Work $\ldots \ldots \ldots \ldots$

7.1 Conclusion . . . . . . . . . . . . . . . . . . . . . . . 173

7.2 Future Work . . . . . . . . . . . . . . . . . . . 176 
References . . . . . . . . . . . . . . . . . . . . 179 



\section{List of Figures}

1.1 The desired $\mathbf{T}_{r e q}$ and the projected $\mathbf{T}$ torques for a given ambient magnetic field B vector. . . . . . . . . . . . . . . . . . . 6

1.2 Magnetic rods operate at alternate times with the magnetometers, to reduce noises on the latter. . . . . . . . . . . . . . . . . . . . . . . 10

1.3 Time structure for the intermittent process of the magnetic rods controller command and the related time terminology definitions . . . .

2.1 NED, ECEF, and ECI frames . . . . . . . . . . . . . . . 27

$3.1 \quad$ Desired torques and their projection for a given ambient magnetic field vector for the $\boldsymbol{\omega}, \mathrm{B}$-dot and the TOC B-dot control laws. . . . . . . 50

$3.2 \quad$ Angular velocity history w.r.t inertial frame . . . . . . . . . . 58

3.3 Absolute angular velocity Comparison f . . . . . . . . . . . . . 58

3.4 TOC B-dot $t_{d e t}$ versus $\chi$ change $\ldots \ldots \ldots$. . . . . . . . 59

3.5 TOC B-dot $\mathbf{t}_{o n}$ versus $\chi$ change $\ldots \ldots \ldots$. . . . . . . . . 59

3.6 Improvement percentage in $t_{d e t}$ for the TOC B-dot at $\chi=1 e^{-6}$ for 200 different initial small $\boldsymbol{\omega}$. . . . . . . . . . . . . . . . . . . . 
3.7 Improvement percentage in $t_{d e t}$ for the TOC B-dot at $\chi=1 e^{-6}$ for 200 different initial highw. . . . . . . . . . . . . . . 61

$3.8 t_{\text {det }}$ reduction percentage of $t_{\text {det }}$ for 200 different initial small $\boldsymbol{\omega}$. Histogram represents results of fig. $[3.6$. . . . . . . . . . . . . . . 62

$3.9 t_{\text {det }}$ reduction percentage of $t_{\text {det }}$ for 200 different initial highw. Histogram represents results of fig. $\mid 3.7$. . . . . . . . . . . . . . . 62

3.10 Improvement percentage in $\mathbf{t}_{\text {on }}$ for the TOC B-dot at $\chi=1 e^{-6}$ for 200 different initial small $\omega$. . . . . . . . . . . . . . . 63

3.11 Improvement percentage in $\mathbf{t}_{o n}$ for the TOC B-dot at $\chi=1 e^{-6}$ for 200 different initial highw. . . . . . . . . . . . . . . 63

3.12 Power saving percentage for 200 different initial small $\boldsymbol{\omega}$. Histogram

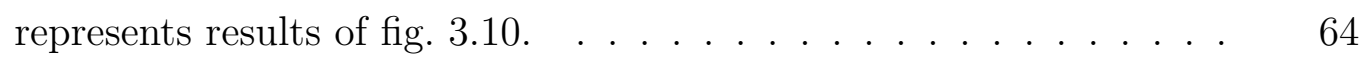

3.13 Power saving percentage for 200 different initial highw. Histogram represents results of fig. $\mid 3.11.] \ldots \ldots . \ldots . \ldots 64$

3.14 Magnetic rods $\mathbf{t}_{\text {on }}$ for B-dot and TOC B-dot control laws for the X, Y, and $\mathrm{Z}$ axes and the mean in orbit period scale. . . . . . . . . . . . . 65

$4.1 \quad$ Angular velocities magnitude history $\ldots$. . . . . . . . . . . . . . 89

$4.2 \quad$ History of the dipole moment magnitude . . . . . . . . . . . . . . . 89

$4.3 \quad$ Angular velocity history for the PMP B-dot controller . . . . . . . . 90

4.4 Histogram represents the $t_{\text {det }}$ reduction percentage for the PMP $\boldsymbol{\omega}$ law

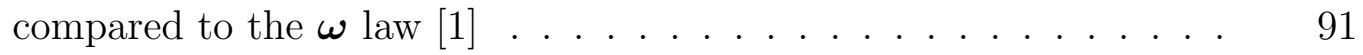


4.5 Histogram represents the $\mathbf{E}$ reduction percentage for the PMP $\boldsymbol{\omega}$ law compared to the $\boldsymbol{\omega}$ law $[1] \ldots \ldots 1$

4.6 Histogram represents the $t_{\text {det }}$ reduction percentage for the PMP B-dot law compared to the B-dot law[1] . . . . . . . . . . . . . . . . . . 92

4.7 Histogram represents the $\mathbf{E}$ reduction percentage for the PMP B-dot law compared to the B-dot law [1] . . . . . . . . . . . . . . . . . . . 92

4.8 Detumbling time for the B-dot law [1] and the PMP B-dot law for different altitudes at orbits of $6^{\circ}$ inclination angle . . . . . . . . . . 93

4.9 Electric energy for the B-dot law [1] and the PMP B-dot law for different altitudes at orbits of $6^{\circ}$ inclination angle . . . . . . . . . . . . 93

4.10 Detumbling time for the B-dot law [1] and the PMP B-dot law for different magnetometer noise standard deviation $\sigma_{m a g}$. . . . . .

4.11 Electric energy for the B-dot law [1] and the PMP B-dot law for different magnetometer noise standard deviation $\sigma_{m a g}$. . . . . . . . .

4.12 Improvement of detumbling time and electric energy consumption versus orbital altitude and inclination . . . . . . . . . . . . . . . . 95

4.13 Histogram represents the $t_{\text {det }}$ reduction percentage for the PMP B-dot law Eq.(4.43) compared to the TOC B-dot law Eq.(3.8) . . . . . . 97

4.14 Histogram represents the $\mathbf{E}$ reduction percentage for the PMP B-dot law Eq.(4.43) compared to the TOC B-dot law Eq.(3.8) . . . . . . 
5.1 The magnetic rods operate on a duty cycle, alternating with the magnetometers to avoid high noise on magnetometers. . . . . . . . . . . 101

$5.2 \quad$ Log-Log L-curve of the norm of the regularized term and the norm of residual term . . . . . . . . . . . . . . . . . . . . . . . . . 108

5.3 Spacecraft attitude error history in terms of principal rotation error for different constant $\mathbf{T}_{D} . \ldots \ldots \ldots \ldots \ldots$

5.4 Spacecraft angular velocity magnitude history for different constant

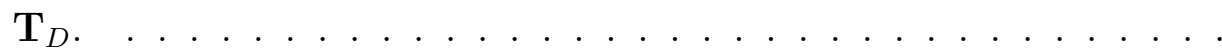

5.5 Spacecraft attitude error history in terms of principal rotation error for different duty cycles. . . . . . . . . . . . . . . . . . . . . . 120

5.6 Spacecraft attitude error history in terms of principal rotation error for different proportional gain $k_{p}$. . . . . . . . . . . . . . . 120

5.7 Spacecraft angular velocity history. . . . . . . . . . . . . . . . . . . 124

5.8 Spacecraft quaternion history over time (orbits). . . . . . . . . . . . 124

5.9 The $\epsilon$ parameter effect on the normalized maneuver time . . . . . . 125

5.10 The $\epsilon$ parameter effect on the normalized $\mathbf{t}_{\text {on }}$. . . . . . . . . . . 125

5.11 The $\epsilon$ parameter effect on the CC of the computed $\mathbf{B}_{\text {sdo. }}$. . . . . . 127

5.12 The $\epsilon$ parameter effect on the SI of the computed $\mathbf{B}_{s d o}$. . . . . . . 127

5.13 The $\epsilon$ parameter effect on the NRMSE of the computed $\mathbf{B}_{\text {sdo. }}$. . . 127

5.14 The $\epsilon$ parameter effect on the normalized computational load . . . . 127 
5.15 Maneuver time reduction percentage for 2,400 Monte Carlo runs for 240 different initial conditions. . . . . . . . . . . . . . . . . . . . . . 130

5.16 Power saving percentage for 2,400 Monte Carlo runs for 240 different initial conditions. . . . . . . . . . . . . . . . . . . . . . 130

5.17 Scatter Index for 2,400 Monte Carlo runs for 240 different initial conditions. . . . . . . . . . . . . . . . . .

5.18 The NRMSE for 2,400 Monte Carlo runs for 240 different initial conditions. . . . . . . . . . . . . . . . .

5.19 Attitude error in principal rotation angle $\Phi, \alpha$ and $\lambda$ histories for $1^{\text {st }}$ maneuver.

5.20 Angular velocities from unbiased gyroscope measurements and the EKF output for $1^{\text {st }}$ maneuver.

5.21 Magnetic field history for $1^{\text {st }}$ maneuver. . . . . . . . . . . . . . . . . 139

5.22 Dipole moment history for $1^{\text {st }}$ maneuver. . . . . . . . . . . . . . 139

5.23 Torque history for $1^{\text {st }}$ maneuver . . . . . . . . . . . . . . . . . 139

5.24 Magnetic field history for $2^{\text {nd }}$ maneuver. . . . . . . . . . . . . . . 140

5.25 Dipole moment history for $3^{\text {rd }}$ maneuver. . . . . . . . . . . . . . . 140

6.1 In the reference ADCS algorithm, the magnetic rods operate on a duty cycle, alternating with magnetometers to avoid high noise on the lat-

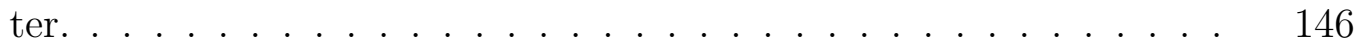

6.2 The proposed magnetic rods operation. . . . . . . . . . . . . . . . . 147 
6.3 The relation between $\alpha$ and each of $\lambda$ (the left vertical axis) and $\eta$ (the right vertical axis). The angles $\lambda$ and $\eta$ are varied and the corresponding change in $\alpha$ is computed. . . . . . . . . . . . . . . . . . . . . . . 152

6.4 Spacecraft quaternion history over time. . . . . . . . . . . . . . . 160

6.5 Spacecraft angular velocity and principal rotation angle error. . . . 160

6.6 Ambient magnetic field history over time. . . . . . . . . . . . . . . . 161

6.7 Normalized Man. time, PC and CL. . . . . . . . . . . . . . . . . . . 161

6.8 CC, SI and NRMSE of the $\mathbf{B}_{\text {sdo }}$ vector. . . . . . . . . . . . . . . . . 162

6.9 Bias, MAE and RMSE of the $\mathbf{B}_{s d o}$ vector. . . . . . . . . . . . . . . 162

6.10 Maneuver time reduction percentage. . . . . . . . . . . . . . 164

6.11 Power consumption reduction percentage. . . . . . . . . . . . . . 164

6.12 Normalized computational load versus normalized maneuver time. . 164

6.13 The mean of the RMSE, MAE and Bias at $\epsilon=6$. . . . . . . . . . . 164

6.14 The mean of the CC, SI and NRMSE at $\epsilon=6$. . . . . . . . . . . . 165

6.15 Normalized maneuver time for the proposed algorithm, $\epsilon=10$. . . 165

6.16 Normalized power consumption for the proposed algorithm, $\epsilon=10 . \quad 167$

6.17 Normalized computational load for the proposed algorithm, $\epsilon=10$. 167

6.18 Angular velocities history from unbiased gyroscope measurements and the EKF output for $1^{\text {st }}$ maneuver. . . . . . . . . . . . . . . . . 169

6.19 Magnetic field history in the X direction for $1^{\text {st }}$ maneuver. . . . . . 169

6.20 Magnetic field history in the Y direction for $1^{\text {st }}$ maneuver. . . . . . 169 
6.21 Magnetic field history in the $\mathrm{Z}$ direction for $1^{\text {st }}$ maneuver. . . . . . 169

6.22 CC, SI and NRMSE of the estimated magnetic field for $1^{\text {st }}$ maneuver. $\quad 170$

6.23 Bias, MAE and RMSE of the estimated magnetic field for $1^{\text {st }}$ maneuver. .............................. 170

6.24 Angular velocities history from unbiased gyroscope measurements and the EKF output for $2^{\text {nd }}$ maneuver. . . . . . . . . . . . . . . . . 170

6.25 Magnetic field histories for the $2^{\text {nd }}$ maneuver. . . . . . . . . . . . . 170 



\section{List of Tables}

3.1 Spacecraft and orbital parameters . . . . . . . . . . . . . 56

3.2 Disturbance Parameters . . . . . . . . . . . . . . . . 56

$3.3 \quad$ Efficiency in detumbling and activation times percentage . . . . . $\quad 66$

5.1 Spacecraft and orbital parameters . . . . . . . . . . . . . 118

5.2 Disturbance Parameters . . . . . . . . . . . . . . . 122

5.3 Validation parameters for three maneuvers . . . . . . . . . . . . 140

6.1 Worst-case disturbance torque magnitudes . . . . . . . . . . . . . . 163

6.2 Mean and standard deviation of the six parameters . . . . . . . 165

6.3 Validation parameters for the second maneuvers . . . . . . . . . . 171 



\section{Preface}

Part of the research work in this dissertation is published as follows:

A) Journal Publications:

1. Mohammed A. A. Desouky and Ossama O. Abdelkhalik. "Improved Spacecraft Magnetic Attitude Maneuvering", AIAA Journal of spacecraft and rockets, accessed May 17, 2019, pp. 1-13, 10.2514/1.A34452.

B) Journal Publications Under Review:

1. Mohammed A. A. Desouky and Ossama O. Abdelkhalik. "A New Variant of the B-dot Control for Spacecraft Magnetic Detumbling ", Elsevier Acta Astronautica, submitted March 2019.

2. Mohammed A. A. Desouky and Ossama O. Abdelkhalik. "A Spacecraft Attitude Estimation Algorithm for Efficient Magnetic Attitude Maneuvers", Elsevier Acta Astronautica, submitted June 2019.

3. Mohammed A. A. Desouky and Ossama O. Abdelkhalik. "Time-Optimal Magnetic Attitude Detumbling", AIAA Journal of spacecraft and rockets, major revision requested on September 2019. 
4. Mohammed A. A. Desouky and Ossama O. Abdelkhalik. "Effect of Duty Cycle on Magnetic Attitude Control", AIAA Journal of spacecraft and rockets, to be submitted September 2019.

C) Proceeding/Conference Publications:

1. Mohammed A. A. Desouky and Ossama O. Abdelkhalik. "Efficient B-dot Law for Spacecraft Detumbling", 29th AAS/AIAA Astrodynamics Specialist Conference, (AAS/AIAA 19-665), Portland, Maine, United States, August 12-16, 2019.

2. Mohammed A. A. Desouky and Ossama O. Abdelkhalik. "Efficient magnetic attitude regulation control", 29th AAS/AIAA Astrodynamics Specialist Conference, (AAS/AIAA 19-664), Portland, Maine, United States, August 12-16, 2019.

3. Mohammed A. A. Desouky and Ossama O. Abdelkhalik. "Improved Magnetic Attitude Control", IEEE National Aerospace and Electronics Conference (2019 NAECON), (362), Dayton, OH, United States, July 15-19, 2019.

4. Mohammed A. A. Desouky, Kaushik Prabhu, and Ossama O. Abdelkhalik. "On Spacecraft Magnetic Attitude Control", 2018 Space Flight Mechanics Meeting, AIAA SciTech Forum, (AIAA 2018-0205), 10.2514/6.2018-0205. 
5. Mohammed A. A. Desouky and Ossama O. Abdelkhalik. "Improved Magnetic Attitude Control", 70th International Astronautical Congress, Washington D.C., United States, October 21-25, 2019, accepted for publication.

6. Mohammed A. A. Desouky, Ossama O. Abdelkhalik and Lucia Gauchia "Optimization of Magnetic Attitude Maneuvers", 30th AAS/AIAA Astrodynamics Specialist Conference, Orlando, Florida, United States, January 6-10, 2020, accepted for publication.

\section{D) Paper Ranked in Top Five Papers in The American Astronomical So- ciety Molly K. Macaulay Award:}

Mohammed A. A. Desouky and Ossama O. Abdelkhalik. "A faster B-dot law for small spacecraft", John Glenn Memorial Symposium, Cleveland, OH, United States, July 2019.

F) Invention Disclosures:

1. Invention Disclosure at Michigan Technological University, Magnetic satellite Attitude Control without Magnetometers, Ossama O. Abdelkhalik, Mohammed A. A. Desouky and Kaushik Prabhu, Jun 2017.

2. Invention Disclosure at Iowa State University, ISURF 04873, A faster B-dot control for spacecraft magnetic detumbling, Mohammed A. A. Desouky and 
Ossama O. Abdelkhalik, Jan 2019. 


\section{Acknowledgments}

I would also like to thank my advisor Dr. Abdelkhalik for his academic advice and his constant support and encouragement.

Moreover, I would like to thank Dr. David Miles and Mr. Andrew Howarth for providing the CASSIOPE telemetry data used for verification in this study. 



\section{List of Abbreviations}

\begin{tabular}{|c|c|}
\hline CASSIOPE & CAScade, Smallsat and IOnospheric Polar Explorer \\
\hline ACS & Attitude Control System \\
\hline LEO & low Earth Orbit \\
\hline $\mathrm{EKF}$ & Extended Kalman Filter \\
\hline WMM & World Magnetic Model \\
\hline IGRF & International Geomagnetic Reference Field \\
\hline TOC & Triple Orthogonality Condition \\
\hline NMPC & Nonlinear Model Predictive Control \\
\hline SV & Secular Variation \\
\hline NED & North East Down frame \\
\hline $\mathrm{ECEF}$ & Earth-Centered Earth-Fixed frame \\
\hline ECI & Earth-centered Inertial frame \\
\hline FoI & Frame of Interest \\
\hline PMP & Pontryagin Minimum Principle \\
\hline NTOCs & Normal Time-Optimal Control system \\
\hline $\mathrm{CC}$ & Correlation Coefficient \\
\hline SI & Scatter Index \\
\hline RMSE & Root Mean Square Error \\
\hline
\end{tabular}

xxix 
NRMSE

MAE

MEKF

ADCS
Normalized Root Mean Square Error

Mean Absolute Error

Multiplicative Extended Kalman Filter

Attitude Determination Control System 


\section{Nomenclature}

$\mathbf{q}$ Quaternion, where $\mathbf{q}_{v}$ and $q_{0}$ are the vector part and the scalar part

$\boldsymbol{\omega} \quad$ Spacecraft angular velocity $[\mathrm{rad} / \mathrm{sec}]$

$[R(\mathbf{q})]$ Rotation matrix extracted from the quaternion

$[I] \quad$ Spacecraft diagonal tensor matrix $\left[\mathrm{kg} \cdot \mathrm{m}^{2}\right]$

T Applied torque on spacecraft $[\mathrm{Nm}]$

$\mathbf{T}_{g g} \quad$ Gravity gradient torque $[N m]$

$\mathbf{T}_{\text {aero }}$ Aerodynamics torque $[\mathrm{Nm}]$

$\mathbf{T}_{s r} \quad$ Solar radiation torque $[\mathrm{Nm}]$

$\mathbf{T}_{r d s} \quad$ Residual torque due to residual magnetic field $[\mathrm{Nm}]$

$\mathbf{T}_{d} \quad$ Un-modeled disturbance torque on spacecraft $[\mathrm{Nm}]$

M Magnetic rods dipole moment vector $\left[A m^{2}\right]$

B True ambient magnetic field vector in body frame [Tesla]

$\mathbf{N} \quad$ Number of windings vector in the magnetic coil

$\mathbf{i}_{c} \quad$ Current driven vector inside the magnetic rods $[A]$

A Cross section area vector of the magnetic coil $\left[\mathrm{m}^{2}\right]$

$M_{\max }$ Maximum allowable dipole moment magnitude $\left[A \mathrm{~m}^{2}\right]$

$\mathbf{R}_{0} \quad$ Earth center to spacecraft mass center position vector $[\mathrm{Km}]$

$\Omega \quad$ The orbital rate $[\mathrm{rad} / \mathrm{sec}]$ 
$\mathbf{R}_{m p} \quad$ Spacecraft mass center to pressure center position vector $[m]$

$\rho \quad$ Atmospheric density $\left[K g \cdot m^{3}\right]$

$C_{D} \quad$ Aerodynamic drag coefficient

$\mathbf{v}_{s c} \quad$ The translational velocity vector of the spacecraft $[\mathrm{m} / \mathrm{sec}]$

$\mathbf{A}_{s c} \quad$ Spacecraft surface area that is orthogonal $\mathbf{v}_{s c}\left[\mathrm{~m}^{2}\right]$

$C_{r k} \quad$ Constant used to specify the outer material of the satellite

$\mathbf{R}_{S s} \quad$ Spacecraft mass center to Sun's mass center vector $[K m]$

$F_{\text {solar }}$ The total solar irradiance $\left[w / m^{2}\right]$

c The speed of light $\left[\mathrm{m} / \mathrm{s}^{2}\right]$

$\mathbf{M}_{r d s} \quad$ Residual magnetic dipole moment vector $\left[A m^{2}\right]$

$J \quad$ Performance index or cost function

$t_{0}, t_{f} \quad$ Initial and final times $[\mathrm{sec}]$

$H \quad$ The Hamiltonian scalar

$\boldsymbol{\lambda}$ The co-state variable or Lagrange multiplier vector

$\overline{\boldsymbol{\omega}} \quad$ Geomagnetic field rotation velocity around the $\operatorname{Earth}[\mathrm{rad} / \mathrm{sec}]$

$\boldsymbol{\omega}_{s s} \quad$ Steady sate angular velocity error vector

$w_{k} \quad$ Weight where $k=1, . ., y$

$T_{s} \quad$ Cycle period or the sampling period of the measurements [sec]

$\delta \quad$ Duty cycle

E The consumed electric energy vector [Watt.Hrs]

V Voltage vector for the magnetic rods [volt $]$ 
$k_{i} \quad$ Controller gains, where $i=w, p, d$

$\zeta_{m} \quad$ Orbit inclination [degree]

$\sigma_{m a g}$ Standard deviation of magnetometer noise [Tesla]

$\mu \quad$ Mean value

$h \quad$ Orbital altitude $[\mathrm{km}]$

$\mathbf{t}_{\text {on }}$ Magnetic rods activation time vector

$\bar{\delta} \quad$ Duty cycle for the proposed algorithm

$f_{c} \quad$ Control command update frequency

$\alpha \quad$ Regularization parameter

$\sigma_{i} \quad$ Singular values of the matrix $[M]_{x}, i=1,2,3$

$\kappa \quad$ Curvature radius of the L-curve

$\Delta T \quad$ The residual torque vector

$f_{\omega} \quad$ Gyro measurement frequency 



\section{Abstract}

This study focused on providing applicable control solutions for spacecraft magnetic attitude control system. Basically, two main lines are pursued; first, developing detumbling control laws and second, an improvement in the three-axis attitude control schemes by extending magnetic rods activation time.

Spacecraft, after separation from the launching mechanism, experiences a tumbling phase due to an undesired angular momentum. In this study, we present a new efficient variant of the B-dot detumbling law by introducing a substitute of the spacecraft angular velocity, based on the ambient magnetic field data. This B-dot law preserves the orthogonality, among the applied torque, dipole moment and magnetic field vectors. Most of the existing variants of the B-dot law in the literature don't preserve this orthogonality. Furthermore, the problem of minimum-time spacecraft magnetic detumbling is revisited within the context of optimal control theory. Two formulations are presented; the first one assumes the availability of the angular velocity measurements for feedback. The second formulation assumes the availability of only the ambient magnetic field measurements in the feedback; the latter is considered another optimal-based B-dot law. A reduction in detumbling time is fulfilled by the proposed laws along with less power consumption for the proposed B-dot laws. 
In magnetic attitude maneuvers, magnetic rods and magnetometers usually operate alternatively, to avoid the magnetic rods' noise effect on magnetometers measurements. Because of that, there will be no control authority over the spacecraft during the magnetometer measurement period. Hence longer maneuver times are usually experienced. In this study, a control scheme that enables the extension of the magnetic rods' activation time is developed, regardless of the attitude control law. The key concept is replacing the real magnetic field measurement by a pseudo measurement, which is computed based on other sensors measurements. By applying a known command to the spacecraft and measuring the spacecraft response, it is possible to compute the ambient magnetic field around the spacecraft. The system mathematical singularity is solved using the Tikhonov regularization approach. Another developed approach estimats the magnetic field, using a relatively simple and fast dynamic model inside a Multiplicative Extended Kalman Filter. A less maneuver time with less power consumption are fulfilled. These control approaches are further validated using real telemetry data from CASSIOPE mission.

This dissertation develops a stability analysis for the spacecraft magnetic attitude control, taking into consideration the alternate operation between the magnetic rods and the magnetometers. It is shown that the system stability degrades because of this alternate operation, supporting the proposed approach of extending the operation time of the magnetic rods. 


\section{Chapter 1}

\section{Introduction}

This dissertation describes the design and analysis of the detumbling control laws for small spacecraft in low earth orbit using magnetic rods only. A novel gain-based B-dot law is developed. Moreover, the problem of minimum-time spacecraft attitude detumbling within the context of optimal control theory is developed and solved.

Furthermore, novel control schemes are developed for three-axis attitude control. These control schemes enable the spacecraft to carry out attitude maneuver in less time with less power consumption and improve the system stability. These control scheme are further validated via real telemetry data from the CAScade, Smallsat and IOnospheric Polar Explorer (CASSIOPE) mission. 


\section{$1.1 \quad$ Background}

Attitude Control Systems (ACSs) play an essential role in almost every task during spacecraft's lifetime, such as Apogee Booster Motor (ABM), normal operation, survival mode, and de-orbiting at the end of the spacecraft's mission. Whereas numerous studies on attitude control actuators have been conducted over years, a particular interest is in magnetic rods.

Magnetic rods, and sometimes called magnetorquers or magnetic actuators, are featured by several interesting properties such as simple design and operation (there is no need for moving parts or plumbing,) low weight, energy efficient, long life time with high reliability, and their ability to modulate smoothly the control torque, which does not induce unwanted coupling with the flexible mode (which is the case when using thrusters) [1-3]. These advantages of magnetic rods attract the designers to consider them as favorable candidates for performing three-axis attitude and detumbling maneuvers for small spacecraft [4].

A survey of worldwide pico- and nanosatellite missions highlights that the magnetic attitude control is very popular in nanosatellites either passive or active. About $40 \%$ of the nanosatellites have magnetic rods for active magnetic attitude control [5]. They are especially suitable in practice in low Earth Orbit (LEO) satellites [2, 3]. Magnetic 
rods are by far the cheapest, however their drawbacks include poor accuracy and even instantaneous under actuation [6]. Yet, they are widely studied for satellite attitude control as described in many references such as [6-25].

Magnetic rods have been used as a primary actuator for detumbling maneuvers [2631], and also for attitude acquisition maneuvers [32, 33] with gravity gradient spacecraft [34] and the momentum biased spacecraft [2, 35 38], whether the goal is earth pointing [3, 16] or inertial pointing [10, 11]. For spacecraft that are actuated mainly by reaction wheels, magnetic rods can still be used as secondary actuators for momentum management [2, 4].

In conjunction with using the magnetic rods for magnetic attitude control, magnetometers are usually used for measuring the spacecraft external or ambient magnetic field. Magnetometers are featured as relatively inexpensive equipment and reliable, which can be easily redundant if necessary. The magnetic field measurements are sometimes used to compute the control command for magnetic rods. For example, in detumbling maneuvers, the B-dot control law is widely used to compute the control command, where the time rate of change of the ambient magnetic field is used [39]. For three-axis attitude control, magnetometer measurements is a main component in the ACS as can be found in several references such as [37, 38, 40, 49].

In addition, magnetometer measurements can also be used in attitude determination. In such case, each three-axis magnetometer measurement provides only two axes of 
attitude information. Therefore, an Euler model for spacecraft attitude propagation between measurements is usually used to solve the lack of attitude determination from a single frame [14]. There are several approaches that can be used for the latter purpose. Psiaki et al. [50] proposed an Extended Kalman Filter (EKF) for attitude, rate and constant disturbance torque estimation based on magnetic field measurements and their time derivatives. Tortora et al. [51] proposed a fast angular rate estimation scheme using magnetometer readings, assuming that the inertial ambient magnetic field vector does not significantly change during the short sampling time. An analytic approach is used in [51] that does not require attitude information. Humphreys et al. [14] presented a magnetometer-based filter and smoother for estimating the attitude, rate, and boom orientations for a spinning spacecraft that has wire booms. Abdelrahman and Park employed the Sigma-Point Kalman Filter for spacecraft three-axis attitude and rate estimation based on magnetometer measurements and their time derivatives. This filter's capability in estimating the attitude is better than 5 deg, and the rate error is on the order of $0.03[\mathrm{deg} / \mathrm{s}]$ in each axis [52].

In the above cited studies, the magnetometer readings are compared with the propagated values from a high order magnetic field model, such as the World Magnetic Model (WMM) or the International Geomagnetic Reference Field (IGRF) to estimate the spacecraft attitude. Another usage of these models is magnetometers calibration 53 56]. 


\subsection{Magnetic Attitude Control Challenges}

Despite the discussed advantages of magnetic attitude control systems, several challenges, whether natural or raised from design, usually limit the attitude control system capabilities. This section is dedicated to discuss these challenges. Along with some of the most common proposed solutions in the literature. These challenges will be categorized as magnetic rods' challenges in the first subsection and magnetometer's challenges in the remaining two subsections as follows.

\subsubsection{Magnetic Attitude Control Singularity}

Magnetic attitude control systems are usually under-actuated. This can be attributed to the fact that the magnetic rods operate on the basis of the interaction between current-driven magnetic coils and the ambient magnetic field to generate the torque [8, 9]. However, the torques are constrained to remain in the plane orthogonal to the ambient magnetic field vector. Therefore, the three axis magnetic control is only possible if the spacecraft orbit sees a variation of the ambient magnetic field that is sufficient to guarantee the stability and controllability of the spacecraft [2, 57], which is usually possible in inclined orbits. In addition to the ambient magnetic field variability, Yang [45] pointed out that there are additional constraint condition on 


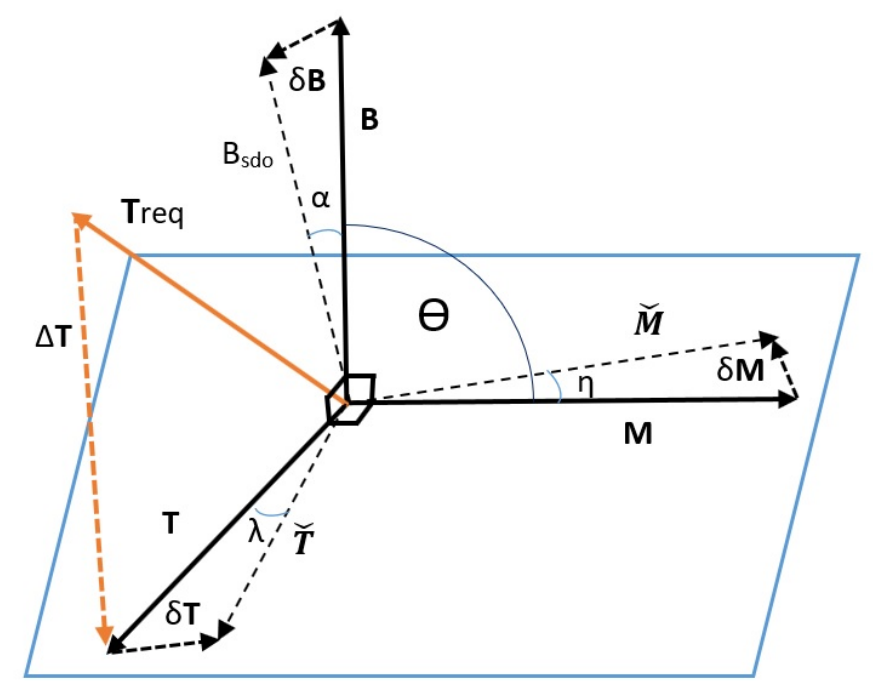

Figure 1.1: The desired $\mathbf{T}_{r e q}$ and the projected $\mathbf{T}$ torques for a given ambient magnetic field $\mathbf{B}$ vector.

the spacecraft inertia tensor to guarantee the controllability, when the problem is approximated by a linear time-varying system.

The control task for such under-actuated systems is more challenging [58]. The spacecraft required torque $\mathbf{T}_{r e q}$, which is computed using the designed control law, in general is not in the plane that is orthogonal to the ambient magnetic field vector $\mathbf{B}$. The required torque vector $\mathbf{T}_{r e q}$ has infinity projection vectors on the plane that orthogonal to the ambient magnetic field vector B. However, intuitively, the projected vector that leads to the minimum losses (minimum maneuver time and minimum power consumption) for this $\mathbf{T}_{r e q}$ is the one that preserves the minimum residual torque. The most common method to mitigate this problem is by using projection-based control technique. Where, magnetometer measurements are used to ensure that the computed dipole moment vector $\mathbf{M}$ results in a torque vector $\mathbf{T}$ that is in the orthogonal 
plane to the ambient magnetic field vector. This is done in a way to minimize the residual torque vector $\Delta \mathbf{T}$ between the required $\mathbf{T}_{r e q}$ and the computed torque $\mathbf{T}$ vectors, see fig 1.1 .

\subsubsection{Magnetometer errors}

Three-axis magnetometer measurements may be corrupted by both constant and time-varying errors [53, 54, 59]. These errors are due to manufacturing tolerances and the surrounding environment as summarized below.

\subsubsection{Manufacturing Tolerance Errors}

The manufacturing tolerance errors include:

1. Null Shift Errors, also known as DC offset or zero bias, are a constant offset that shifts the output of the sensor. This error will result in a constant magnetometer bias, due to magnetometer manufacturing materials.

2. Scale Factor Errors are caused by uncertainty in the constant of proportionality between the local magnetic field (sensor input) and the sensor output.

3. Non-Linearity Error includes all the deviation from a linear relationship 
between the sensor input and output.

4. Non-orthogonality Error is the deviation from orthogonal sensor axes. It can be caused by manufacturing error.

5. Sensor noise is the stochastic component of the sensor output. It is assumed here in this study as zero mean Gaussian distribution [59].

\subsubsection{Surrounding Environment Errors}

The surrounding environment errors include:

1. Soft Iron Materials Error is due to the generated magnetic field in response to externally applied magnetic field. This results in a magnetometer bias, yet the magnitude of the fields produced by soft iron materials are insignificant compared to the ambient field. Soft iron materials also cause a scale factor error.

2. Hard Iron Error, typically, is caused by unwanted magnetic fields near the magnetometer due to ferromagnetic (hard iron) materials. This error will result in an offset between the ambient and measured magnetic fields and can be parametrized by a constant bias in each magnetometer axis.

3. Non-Orthogonality Error can also caused by the effect of the surrounding 
environment such as thermal or mechanical strain.

4. Time-Varying Biases caused by the generated magnetic field due to current driven through the spacecraft nearby electronics especially the magnetic rods. These biases are the most dominate errors and the available design solutions, to lessen these biases, in the literature, add to the system challenges as will be discussed in the following.

Magnetometer measurements degrade because of the above mentioned errors, which traditionally limit their utility in satellites. One way to fix this problem is to use a boom to provide physical separation between the magnetometer and the satellite [60]. In other situations, the ambient magnetic field measurements are collected at intermittent times, which are selected to be different from the times in which the magnetic rods (coils) are actuated (a duty cycle for the rods). This is carried out by switching the magnetic coils on and off, so that the magnetometer is not affected by the magnetic field of the magnetic coils [24, 61 63], see fig. 1.2. This approach, however, could lead to a degradation in the performance in terms of the maneuver time, electrical power consumption [62] and steady state error for attitude acquisition maneuvers.

For space scientific missions that are dedicated for measuring the earth magnetic field, such as the CAScade Smallsat and IOnospheric Polar Explorer (CASSIOPE) mission, both a boom is used to put the magnetometers away from the rest of the 


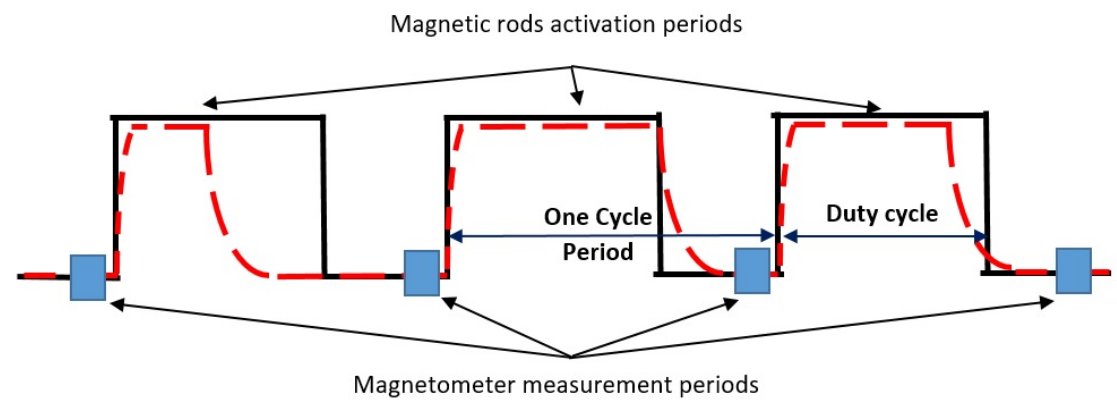

Figure 1.2: Magnetic rods operate at alternate times with the magnetometers, to reduce noises on the latter.

satellite electronic components, and a intermittent operation for the magnetic rods is implemented [60]. Such a solution leads to more complex structures, more weight, and increased system complexity due to the boom deployment mechanism. For small satellites or low cost missions that use magnetic rods, the magnetic rods are usually operated in a intermittent fashion on a duty cycle, as shown in fig. 1.2 .

Figure 1.3 shows the cycle of activation for the magnetic rods and the magnetometer measurements. Starting by activating the magnetic rods, the rise period is the time required by the system to rise the current or equivalently the generated dipole moment from zero to the maximum value for constant current. While in the fall period, the dipole moment or the current will go down to zero. A desaturation period includes the fall period and the separation between the fall period end and the measurements period; this desaturation period's function, as its name implies, is to desaturates the generated magnetic field from the magnetic rods in order to eliminate their effect on the ambient magnetic field measurements. The rise time and desaturation period 


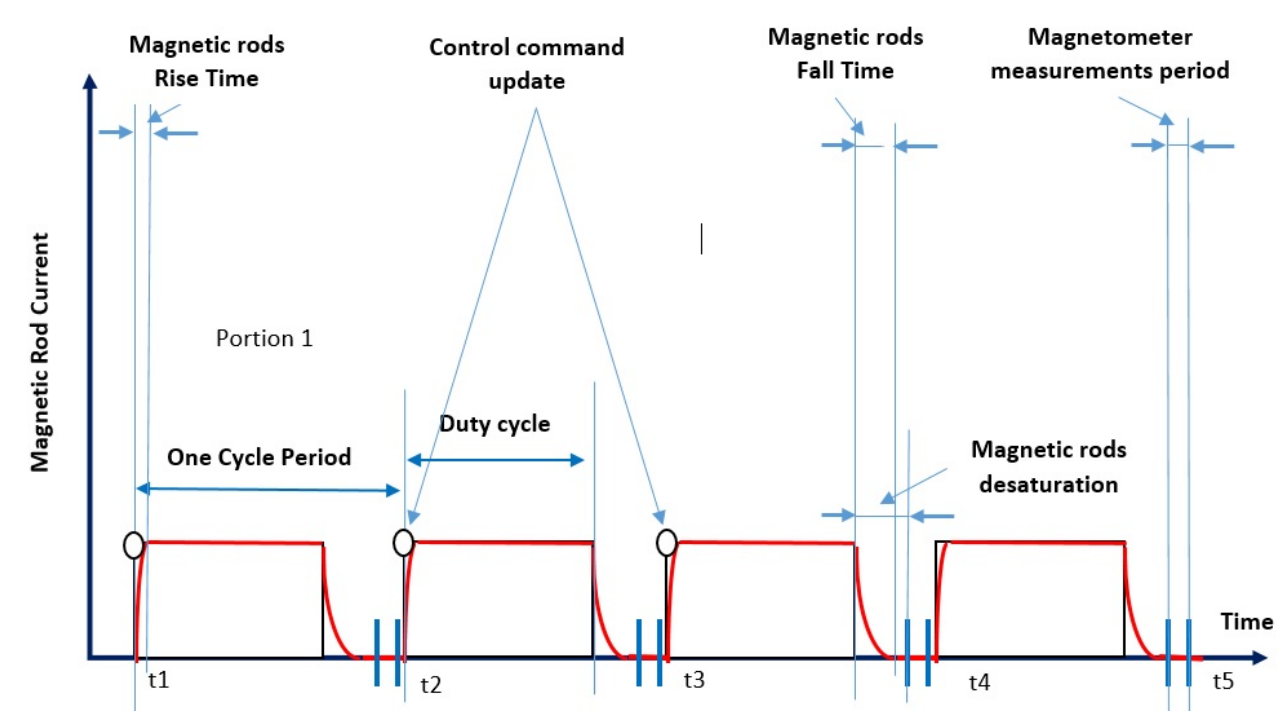

Figure 1.3: Time structure for the intermittent process of the magnetic rods controller command and the related time terminology definitions

needed by the magnetic rod is constant and is a manufacturer specification.

The duty cycle is the percentage of the cycle (sampling time $T_{s}$ ) that defines the maximum activation time of the magnetic rods. For example, a 0.6 duty cycle $(\delta)$ means that the maximum activation time for the magnetic rods for the entire maneuver time is $60 \%$, where $0<\delta<1$. Ignoring such an important factor in the analysis, design and simulation may give erroneous results. The activation time vector $\mathbf{t}_{\text {on }}$ for the three magnetic rods inside the duty cycle for the constant current case is computed as follows:

$$
\mathbf{t}_{\text {on }}=\delta T_{s} \frac{|\mathbf{M}|}{M_{\max }}
$$

where $\mathbf{M}_{\max }$ is the maximum allowable dipole moment. The commanded current 
direction in each axis has the same sign as the corresponding component of the $\mathbf{M}$ vector. Equation (1.1) enables the computation of the width of the modulated signal from the controller to the actuator. The summation of the $\mathbf{t}_{\text {on }}$ vectors over the entire maneuver time will be used later as a measure for the magnetic rods activation time or power consumption during the simulations.

\subsubsection{Magnetometer Calibration}

High order models, such as (WMM and IGRF), can be used for magnetometer calibration [53, 54]. These models are usually around the $10^{\text {th }}$ order or more. For example, the WMM consists of a $12^{\text {th }}$ order spherical-harmonic main (i.e., core-generated) field model comprised of 168 spherical-harmonic Gauss coefficients. The Secular Variation (SV) is also considered since the Earth's liquid-iron outer core that contributes to most the Earth's magnetic field intensity changes distinctively from year to year. This SV is calculated by a linear SV model in the WMM. However, due to some nonlinear variations, the WMM must be updated every 5 years; and the next update was scheduled to take place in 2020 . Due to unknown reasons, as of the time this dissertation is being written, the North Pole movement rate has increased more than the predicted rate, heading from Canada to Siberia. Therefore, in the meantime, the accuracy of the WMM is questionable; and may lead to unacceptable accuracy for navigation purposes. As a result, the WMM is now planned to be updated during 
2019 [64, 65]. This triggered the National Geospatial-Intelligence Agency to carry out a global competition (\$1.2M NGA's MagQuest) to inspire scientists to find new ways to ensure that there is reliable, sustainable geomagnetic data to feed into the World Magnetic Model 11 .

\subsection{Magnetic Attitude Control Maneuvers}

This section is dedicated to discuss mainly two attitude maneuvers that can be fulfilled using the magnetic rods; the detumbling maneuver and the three-axis attitude control maneuvers.

\subsubsection{Detumbling Maneuvers}

The goal of the spacecraft detumbling is to bring the spacecraft angular velocity from initial condition, most probably featured by high angular momentum, down to zero angular momentum in the ideal case. This detumbling task is required after separation from the deployment mechanism at the initial phase or after retrieving the spacecraft from energy save mode or survival mode that must turn off the power of the ACS. Failure to detumble the spacecraft sufficiently within the designed time

1 https://www.magquest.com/ 
frame, may lead to uncontrollable spacecraft and/or significant power consumption.

Thus, several studies have focused on this problem searching for a reliable solution with minimum risk. Tight requirements on the reliability of the detumbling actuators and sensors, and on the simplicity of the adopted control law, usually drive the design of the detumbling control system [4]. This usually leads to the use of magnetic rods; as they are considered excellent candidates for driving a detumbling spacecraft along with ambient magnetic field information sensor.

Some magnetic detumbling control approaches require the availability of both the spacecraft angular velocity (measured or estimated such as the work in [51]), along with the ambient magnetic field information. In reference [1], for instance, the spacecraft angular velocity is used in the feedback loop to obtain the required or the designed torque $\mathbf{T}_{r e q}$. This control law is of type projection-based control laws. These control laws preserve the Triple Orthogonality Condition (TOC) between the dipole moment $\mathbf{M}$, the ambient magnetic field $\mathbf{B}$, and the generated spacecraft applied torque $\mathbf{T}$ vectors in the ideal case, see fig 1.1 .

For the gyros, however, there is a limitation of the maximum spacecraft angular velocity that the gyros can sense. Moreover, the gyros suffer low reliability; for example, the Hubble Space Telescope was put into safe hold mode due to the failure of gyros [51] and the technical team were able to recover this situation. 
Other magnetic detumbling control approaches, however, use only the ambient magnetic field measurements to compute the control torque. This is because the magnetic field rate of change is a good indicator of the change in the spacecraft orientation. This detumbling control law is called B-dot law which was first proposed in [26]. This control law depends on computing the derivative of the ambient magnetic field (as can be inferred from the controller name,) in the spacecraft body frame. The ambient magnetic field derivative is then used as an indication of the spacecraft angular velocity with the assumption of high angular velocity. However, there is a degradation in the B-dot controller performance when the angular velocity is low, which is usually the case when the spacecraft is close to being fully detumbled, or if the initial angular velocity happens to be small [66]. Since the development of the first B-dot control, there has been several variants of it, designed for the detumbling maneuver [27 31], and also for attitude acquisition [32, 33].

Few studies address the time-optimal detumbling using only magnetic rods. Avanzini and Giulietti [1] provided a sub-optimal gain expression based on analyzing the closed loop dynamics of the spacecraft angular velocity component that is orthogonal to the earth magnetic field. This gain expression is a function of the orbital inclination, orbital rate, and minimum moment of inertia component. This gain expression is provided for two cases: the first case assumes the availability of the angular velocity measurements, and the second case assumes the availability of only the magnetic field measurements. Juchnikowski et al. [67] derived analytically a time-optimal B-dot law 
gain as a function of the magnitude of the magnetic field and its derivative. However, the derivation is limited only to the case of spacecraft with spherical symmetry of mass distribution. Juchnikowski et al. extended their work, numerically only, and showed that the time-optimal B-dot law gain gives promising results for non-symmetrical satellites [68. Bohm et al. [69] presented a Nonlinear Model Predictive Control (NMPC) approach for spacecraft angular moment damping in the initial acquisition phase. A good performance is achieved at the expense of the computational load. Ahmed et al. [70] proposed NMPC for damping the spacecraft angular velocity that is featured by its low computational load due to the added constraint that terminates the optimization process early, especially when the initial angular velocity is high. This early termination of the optimization process may lead to non-feasible solutions at some times. Liu et al. [71] developed detumbling and attitude acquisition control approaches by combining a B-dot algorithm, a bias momentum algorithm, and a sliding mode control approach, using magnetic rods along with a pitch bias momentum wheel.

On the other hand for the purpose of comparison, for fully actuated spacecraft, aiming at minimizing the detumbling maneuver time, several studies developed control solutions for the time-optimal detumbling maneuvers. Yang and Wu solved the timeoptimal control problem using an iterative procedure for solving the non-linear programming problem [72]. Romano derived an analytic solution for detumbling and nutation cancellation maneuvers for axisymmetric spacecraft [73]. Aghili proposed 
a closed-loop form for this problem taking into account the control effort constraint [74]. Reference [75] provides a more comprehensive survey of reorientation control approaches for fully actuated spacecraft.

\subsubsection{Three-Axis Attitude Control Maneuvers}

The goal of the three-axis attitude control maneuver is to control the spacecraft orientation with respect to a defined frame (such as inertial pointing or earth pointing maneuvers) or another entity like the celestial sphere, certain fields, and nearby objects. This maneuver is required for almost all space missions. It depends on the available onboard measurements and attitude actuators along with the specification of the desired attitude. Several studies in the literature addressed magnetic threeaxis attitude control problems. Two main lines of work for the magnetic three-axis attitude control problem can be classified from the literature[76]:

1. Methods based on averaged models. Where the time-varying dynamics of the magnetically actuated spacecraft is approximated by a time-invariant model using averaging techniques such as [7, 26].

2. Methods based on full periodic models. As the variability of the geomagnetic field is almost time-periodic such as [34, 35, 37]. 
For instance, Silani and Lovera 2] investigated various linear and nonlinear attitude control methods. The former summarizes classical control, optimal periodic control and robust control methods, while the latter explored the problem from a Lyapunov Stability perspective. Reference [2] offers a new approach towards pursuing the attitude control problem using only magnetic actuators based on prediction of parameters; this was accomplished by assuming the knowledge of the magnetic field and computing a feasible projected torque in an optimal sense. Astolfi and Lovera [40] present a magnetic attitude tracking algorithm that needs magnetometers but does not need rate feedback. A low-gain proportional-derivative-like (PD-like) control law is used in [40]. Lovera and Astolfi [42] presents a global magnetic attitude control algorithm using rate feedback in the presence of gravity gradient torque, using ambient magnetic field measurements. Lovera and Astolfi [7] also presented a solution based on static attitude with rate feedback and dynamic attitude feedback using magnetic actuators and sensors, without addressing the issue of magnetic rods noises on the magnetometers. Wang et al. [3] approached the magnetic attitude control problem in two steps. First, designing an outer loop within the nonlinear periodic framework using back stepping for virtual control. Second, designing an inner loop for attitude acquisition and detumbling, to track virtual signals using a sliding mode control. The magnetometer measurements were a fundamental part of the proposed methods; yet the impact of the magnetic rods on the magnetometers was not discussed. Gerhardt and Palo [13] derived a control strategy depending on the previous 
and current time magnetic fields, which are used to determine the induced magnetism in the rod. Ivanov et al. [6] solved the spacecraft attitude control and determination problem with three magnetic rods and three-axis magnetometers; they presented a control scheme that accounts for inertia tensor uncertainty and unknown natural disturbances. Gravdahl [77] derived a three-axis magnetic attitude control using feedback measurements of the magnetic field and the angular velocity, and showed that this control is uniformly globally asymptotically stable.

Magnetometer measurements are also used in feedback control in several algorithms of spacecraft attitude control, as in [11, 16, 22, 23, 29, 34, 36, 42,44, 78, 80]. Furthermore, a Lyapunov-based control laws claimed asymptomatic stability or global stability are developed in [7, 11, 46, 48, 49]. In these algorithms, the above cited works, the control analysis and design assume continuous-time magnetometer measurements, and continuous actuation of the magnetic rods.

In other studies, however, this issue of the noises on magnetometers due to magnetic rods are addressed. For instance, Sugimura et al., in [12], developed a magnetic attitude determination and control algorithm, that uses measurements of only the magnetometers. Two extended Kalman filters are used in [12]; one for estimating the angular velocity from magnetometers data using an analytic predictor approach [51], and the other is for estimating the attitude and angular velocity bias. Sugimura et al. took into account the implementation of the magnetic control torque in an 
intermittent fashion with duty cycle to avoid interference with the magnetometers. Celani [61] modified the control law in [7, 11] taking into account the intermittent actuation of the magnetic rods and magnetometer. However, the total desaturation period was assumed to be zero (non-realistic assumption). Celani [24, 63] further designed inertial pointing three-axis magnetic attitude control law, considering the full desaturation and magnetometer periods, using the state feedback and output feedback, respectively. Celani [24, 63] reported exponential stability for this control law.

The activation of the magnetic rods based on a duty cycle, and the alternate operation of the magnetic rods with magnetometers, leads to an acceptable accuracy of the magnetic field measurements, from the control point of view for low cost missions [60]. However, this duty cycle operation results in extended attitude maneuver times, and may require more activation time of the magnetic rods. This longer activation time for the magnetic rods can be attributed to not taking the duty cycle operation into account when designing the control, or/and to the need to compensate for the more disturbance torques that are encountered when having longer maneuver times, especially at periods when the magnetic rods are deactivated. At these periods, the desaturation and magnetometer periods, there is no control authority over the satellites which will affect the stability as the magnetic rods are off and should considered during design phase. 
The projection-based control laws can be used for pointing maneuvers where the control law attempts to derive the spacecraft near the required target by using the $\mathbf{T}$ instead of $\mathbf{T}_{r e q}$ at every time step, see fig.1.1. Large control command results in large error after each time step. Therefore, a restriction on the control gains is required to derive the spacecraft slowly to the desired attitude. It has been shown that the restriction on the damping part is softer than on the positional part [66]. This leads in general to a poor pointing accuracy. This will be investigated through the stability analysis considering the intermittent actuation of the magnetic rods and magnetometer in section 5.3.1.

\subsection{Contributions of This Dissertation}

The contribution of this dissertation can be summarized as follows:

1. Develop an analytic control law for magnetic detumbling maneuvers, within the context of optimal control theory. Two versions of this analytic control law are developed. The first assumes the measurements of the spacecraft angular velocity. The second one assumes the availability of only the measurements of the ambient magnetic field. Monte Carlo simulations show that these two control laws result in reducing the maneuver time by up to $40 \%$. 
2. Develop a novel variant of the B-dot law that maintains the Triple Orthogonality Condition (TOC) when computing the control torque, unlike most existing variants of the B-dot law. This B-dot law is demonstrated to have significant improvement compared to state-of-the-art B-dot laws, in terms of maneuver time and power consumption.

3. Develop a control schemes that mitigate the impact of alternate operation of the magnetic rods and the magnetometers. These schemes extend the operation periods of the rods, resulting in improved maneuver time and power consumption by the rods. This is achieved by computing the magnetic field instead of measuring it, at some of the cycles.

4. Develop a stability analysis for spacecraft magnetic attitude control systems, taking into account the alternate operation of the magnetic rods and the magnetometers.

\subsection{Outline of This Dissertation}

Chapter 2 starts with the definition of the required reference frames and notation used through the dissertation. A brief discussion and mathematical formulations for the dynamics and kinematics motion is provided next. All the disturbance torques influence the spacecraft in low earth orbit is considered and their models are given. 
A brief overview over the CAScade, Smallsat and IOnospheric Polar Explorer (CASSIOPE) spacecraft hardware configuration, mission and the available telemetry data with its frequency is given in Chapter 2 .

Chapter 3 presents a new variant of the B-dot law for small spacecraft detumbling maneuvers. A discussion from the stability and geometric points of view is provided to highlight the difference between the proposed law and the existing B-dot laws.

Chapter 4 presents an analytic derivation for the optimal control law for magnetic detumbling. A stability analysis is also presented.

Chapter 5 presents a new control scheme that enables longer activation of the magnetic rods for attitude regulation maneuvers.

Chapter 6 presents a control scheme for attitude regulation maneuvers using a relatively simple and fast dynamic model to be used inside Multiplicative Extended Kalman Filter.

Chapter 7 presents the conclusions of this dissertation and the future work. 



\section{Chapter 2}

\section{Spacecraft and environment}

\section{models}

This chapter briefly describes the coordinate reference frames and notation used in this study along with the spacecraft kinetic and kinematic models and the environment models. The material in this section is standard and is provided here for completeness of the presentation.

Futhermore, a brief discussion about The CAScade, Smallsat and IOnospheric Polar Explorer (CASSIOPE) mission. Along with discussion about the real telemetry data given by from the technical team, that is used for validating the proposed three-axis magnetic control schemes in chapters 5 and 6 . 


\subsection{Coordinate reference frames}

This section briefly describes the coordinate reference frames. The following reference frames are used:

1. North East Down frame (NED): The origin of this frame is the satellite center of gravity, the down axis is the direction to the Earth's center and the east is the local east direction [81], see fig 2.1.

2. Earth-Centered Earth-Fixed frame (ECEF): The Z-axis is parallel to the Earth's true north. The $\mathrm{X}$-axis intersects the sphere of the earth at $0^{\circ}$ latitude (the equator) and $0^{\circ}$ longitude (prime meridian in Greenwich). In addition, the Y-axis completes the right-handed orthogonal triad, see fig.2.1.

3. Earth-Centered Inertial frame (ECI). The origin of this frame is in the center of the Earth. This reference frame is denoted $i$, and the earth rotates around its $\mathrm{Z}$-axis. The $\mathrm{X}$-axis points towards the vernal equinox. The $\mathrm{Y}$-axis completes the right-handed triad system, see fig 2.1.

4. Orbit frame: The origin of this frame is the satellite center of mass. Its Z-axis pointed towards the center of the earth. The $\mathrm{X}$-axis is in the plane of spacecraft motion, perpendicular to the Z-axis, and points in the spacecraft's direction of motion for circular orbit. The Y-axis completes the right-handed triad system. 


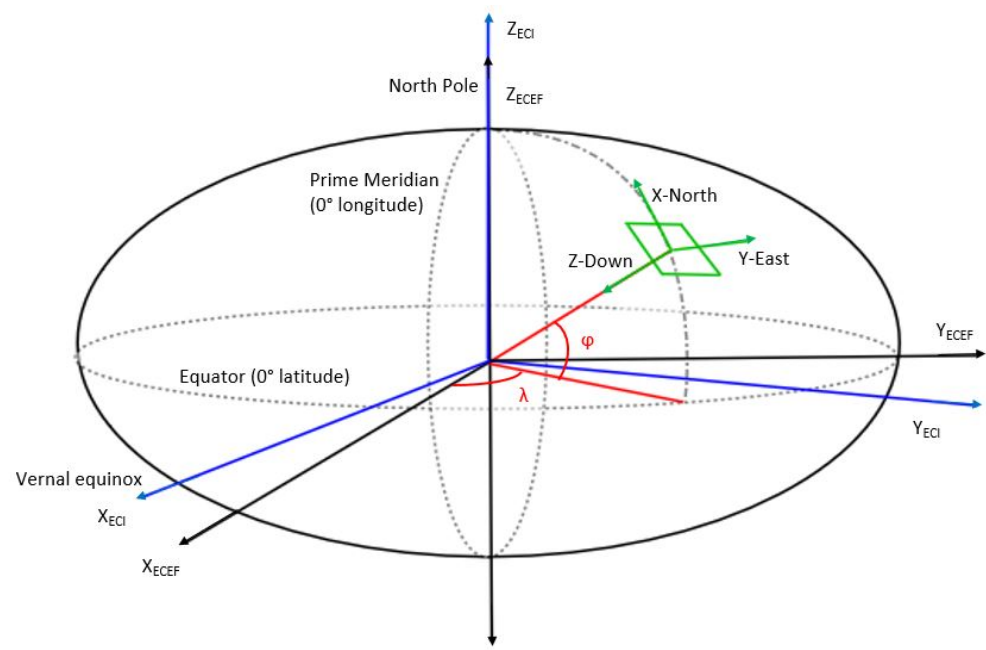

Figure 2.1: NED, ECEF, and ECI frames

The orbit reference frame is denoted $o$.

5. Satellite body frame: The origin of this frame is at the satellite center of mass. This frame is attached to the spacecraft, and its axes are chosen to align with the spacecraft's principal inertia axes. The body frame is denoted $b$.

\subsection{Notation}

In the remainder of this dissertation, [.] represents a matrix, bold symbols represent vector such as $\mathbf{A},[\mathbf{A}]_{x}$ is a skew-symmetric matrix whose elements are the elements of the vector $\mathbf{A}$ that represents the cross product of $\mathbf{A} \times \mathbf{B}=[\mathbf{A}]_{x} \mathbf{B}$, where $\mathbf{B}$ is a vector, $\tilde{\mathbf{B}}$ represents an estimation of the vector $\mathbf{B}, \hat{\mathbf{C}}$ represents a unit vector in the direction of the vector $\mathbf{C}$, and $\overline{\mathbf{D}}$ represents the linearization point of the vector 
function $\mathbf{D}$ (the linearizion process is carried out at $\overline{\mathbf{D}}$.) The ${ }^{i} \mathbf{v}$ represents vector $\mathbf{v}$ defined in the inertial frame $i$. For notation simplification, any vector without a presuperscript is defined in the body $(b)$ frame. The $\boldsymbol{\omega}_{b i}$ represents the angular velocity of the body $(b)$ frame with respect to the inertial $(i)$ frame, expressed in the body (b) frame.

\title{
2.3 Spacecraft rotational kinetic and kinematic
}

\author{
models
}

\subsubsection{Spacecraft kinematics model}

The Euler parameters (quaternion) do not suffer from the singularity problem that is encountered when using the Euler angles in representing spacecraft attitude; and they are computationally less expensive compared to the direction cosine matrix [82]. The spacecraft attitude is here expressed using the four Euler parameters, the quaternion. The attitude kinematics can be written as:

$$
\dot{\mathbf{q}}=\frac{1}{2}\left[\begin{array}{c}
-\mathbf{q}_{v}^{T} \\
q_{4}\left[\mathbf{1}_{3 x 3}\right]+\left[\mathbf{q}_{v}\right]_{x}
\end{array}\right] \boldsymbol{\omega}_{\boldsymbol{b ( F o I})}
$$


where $\mathbf{q} \in \mathbb{R}^{4}$ is the quaternion, and $\mathbf{q}=\left[\begin{array}{ll}q_{0} & \mathbf{q}_{v}\end{array}\right]^{T}$. Let $\mathbf{q}_{v}=\left[\begin{array}{ll}q_{1} & q_{2} \\ q_{3}\end{array}\right]^{T}$. The vector $\mathbf{q}$ satisfies the condition: $\left(\mathbf{q}^{T} \mathbf{q}=1\right)$. The vector $\boldsymbol{\omega}_{\boldsymbol{b ( F o I )}} \in \mathbb{R}^{3}$ is the spacecraft angular velocity of the body frame with respect to the Frame of Interest (FoI), expressed in the body frame, and $\left[\mathbf{1}_{3 x 3}\right] \in \mathbb{R}^{3 \times 3}$ is unity matrix. The quaternion is used to compute the transformation matrix $[R(\mathbf{q})]$ from the Frame of Interest $\left(F_{O} I\right)$ to the body frame:

$$
[R(\mathbf{q})]=\left(q_{0}^{2}-\mathbf{q}_{v}^{T} \mathbf{q}_{v}\right)\left[\mathbf{1}_{3 x 3}\right]+2 \mathbf{q}_{v} \mathbf{q}_{v}^{T}-2 q_{0}\left[\mathbf{q}_{v}\right]_{x}
$$

The transformation matrix $R(\mathbf{q})$ is member of the special orthogonal group of order three as shown in Eq. (2.3). The unit vector norm $\left(\mathbf{q}^{T} \mathbf{q}=1\right)$ constrain maintains the orthogonality condition of the rotation matrix.

$$
[R] \in S O 3=\left\{[R] \mid[R] \in \mathbb{R}^{3 \times 3},[R]^{T}[R]=\left[\mathbf{1}_{3 \times 3}\right], \operatorname{det}([R])=1\right\}
$$

The Frame of Interest $(F o I)$ in this study will be the orbit frame $(o)$ or the inertial frame (i). In case of the $F_{o I}$ is the (o) frame, the spacecraft angular velocity $\boldsymbol{\omega}_{b o}$ can be computed as follows:

$$
\boldsymbol{\omega}_{b o}=\boldsymbol{\omega}_{b i}-[R(\mathbf{q})]^{o} \boldsymbol{\omega}_{o i},
$$

where $[R(\mathbf{q})]{ }^{o} \boldsymbol{\omega}_{o i}=\Omega{ }^{b} \mathbf{J}_{o}$ for circular orbit, ${ }^{b} \mathbf{J}_{o}$ is the unit vector of the Y-axis of the orbit frame, as seen in the spacecraft body frame and $\Omega$ is the orbital rate. 


\subsubsection{Spacecraft kinetics model}

The attitude dynamics of the rigid spacecraft is expressed using Euler's equations as follows [8, 82]:

$$
[I] \dot{\boldsymbol{\omega}}_{b i}=-\boldsymbol{\omega}_{b i} \times[I] \boldsymbol{\omega}_{b i}+\mathbf{T}+\mathbf{T}_{g g}+\mathbf{T}_{a e r o}+\mathbf{T}_{s r}+\mathbf{T}_{r s d}+\mathbf{T}_{d}
$$

where $[I] \in \mathbb{R}^{3 \times 3}$ is the spacecraft inertia matrix, $\mathbf{T}_{g g} \in \mathbb{R}^{3}$ is the gravity gradient torque, $\mathbf{T}_{\text {aero }} \in \mathbb{R}^{3}$ is the aerodynamic torque, $\mathbf{T}_{s r} \in \mathbb{R}^{3}$ is the solar radiation torque, $\mathbf{T}_{r s d} \in \mathbb{R}^{3}$ is the residual magnetic torque due to the residual magnetic field generated by spacecraft electronics including the magnetic rods, $\mathbf{T}_{d} \in \mathbb{R}^{3}$ is the disturbance torque to account for error modeling and unknown sources, $\mathbf{T}_{d}$ is modeled in this study as a zero-mean Gaussian variable. The models of the other torques are summarized in the following section. Hence, the total disturbance torque vector will be as follows:

$$
\mathbf{T}_{D}=\mathbf{T}_{g g}+\mathbf{T}_{\text {aero }}+\mathbf{T}_{s r}+\mathbf{T}_{r s d}+\mathbf{T}_{d}
$$

The control torque on the spacecraft, $\mathbf{T} \in \mathbb{R}^{3}$, is here assumed to be due to only the three magnetic coils aligned with the body frame axes, and hence:

$$
\mathbf{T}=\mathbf{M} \times \mathbf{B}=[\mathbf{M}]_{x} \mathbf{B},
$$


where $\mathbf{B} \in \mathbb{R}^{3}$ and $\mathbf{M} \in \mathbb{R}^{3}$ are the magnetic field and dipole moment vectors, respectively. As evident from Eq. (2.7), it is not possible to generate a torque along the magnetic field vector. The dipole moment is generated by magnetic rods according to this model:

$$
\mathbf{M}=\mathbf{N} \odot \mathbf{A} \odot \mathbf{i}_{c}
$$

where $\mathbf{A} \in \mathbb{R}^{3}$ is the cross section area vector of the magnetic coils, $\mathbf{N} \in \mathbb{R}^{3}$ is the number of windings vector in the magnetic coils, $\mathbf{i}_{c} \in \mathbb{R}^{3}$ is the electric current vector, and $\odot$ denotes component-wise multiplication. There are two options for the electric current: variable current or constant current. In this study, the electric current is assumed variable. The variable electric current vector $\mathbf{i}_{c}$ can be computed as follows:

$$
\mathbf{i}_{c}=\mathbf{M}_{c} \oslash \mathbf{M}_{\max }
$$

where $\oslash$ denotes component-wise division, $\mathbf{M}_{\max } \in \mathbb{R}^{3}$ is the maximum limit dipole moment vector, and $\mathbf{M}_{c} \in \mathbb{R}^{3}$ is the command dipole moment vector. Since $\mathbf{M}_{\text {max }}$, $\mathbf{A}$ and $\mathbf{N}$ in Eqs. 2.8 and 2.9 are constants, it is from now on, for simplicity of presentation, that the command vector is the dipole moment vector $\mathbf{M}$. 


\subsection{Space environment models}

\subsubsection{Orbit propagator}

Spacecraft's orbital position and velocity are propagated in time using a model that accounts for J2 gravitational effect [8, 9]. The J2000 inertial coordinate system is used for the orbit propagation as the reference frame. The output from the orbit propagator is used to determine the spacecraft position with respect to earth and sun, and to compute the aerodynamic density and the geomagnetic field.

\subsubsection{Geomagnetic field model}

The WMM is used in this study to compute the geomagnetic field vector, with Epoch 2015. The geomagnetic field vector is computed by a spacecraft position in North, East and Down frame (NED) frame. An appropriate reference frames are used to complete the transformation of the geomagnetic field parameters to the spacecraft body frame using the transformation matrix that is generated by the quaternion in Eq. (2.2). 


\subsubsection{Gravity Gradient Torque}

The gravity gradient torque $\mathbf{T}_{g g}$ is computed for a circular orbit as follows [8, 82]:

$$
\mathbf{T}_{g g}=3 \Omega^{2}\left(\frac{\mathbf{R}_{o} \times[I] \mathbf{R}_{o}}{\left\|\mathbf{R}_{o}\right\|^{2}}\right)=3 \Omega^{2}\left(\hat{\mathbf{R}}_{o} \times[I] \hat{\mathbf{R}}_{o}\right),
$$

where $\mathbf{R}_{o} \in \mathbb{R}^{3}$ is the position vector from the center of earth to the spacecraft center

of mass, and $\Omega$ is the orbital rate. The gravity gradient torque calculations ignore the gravitational field effect from celestial objects other than the Earth; and the Earth possess a spherical symmetrical mass distribution [8, 82].

\subsubsection{Aerodynamics Torque}

The aerodynamics torque $\mathbf{T}_{\text {aero }}$ is computed as follows [8]:

$$
\mathbf{T}_{\text {aero }}=-\mathbf{R}_{m p} \times\left(\frac{\rho C_{D}}{2}\left\|\mathbf{v}_{s c}\right\|^{2} \hat{\mathbf{v}}_{s c} A_{s c}\right),
$$

where $\mathbf{R}_{m p} \in \mathbb{R}^{3}$ is the vector from the center of mass to the center of aerodynamic pressure of the spacecraft, $\rho$ is the atmospheric density, $C_{D}$ is the aerodynamic drag coefficient, $\mathbf{v}_{s c} \in \mathbb{R}^{3}$ is the translational velocity vector of the spacecraft, and $A_{s c}$ is the orthogonal surface area and calculated using the projection matrix method [83] . 


\subsubsection{Solar Radiation Torque}

The solar radiation torque $\mathbf{T}_{s r}$ is computed as follows [8]:

$$
\mathbf{T}_{s r}=-\mathbf{R}_{m p} \times\left(\frac{C_{r k} A_{s c} \mathbf{R}_{S s} F_{\text {solar }}}{c}\right),
$$

where $C_{r k}$ is a constant used to specify the outer material of the satellite, $\mathbf{R}_{S s} \in \mathbb{R}^{3}$ is the vector from the satellite's center of mass to the Sun's center of mass, $F_{\text {solar }}$ is the total solar irradiance, and $c$ is the speed of light.

\subsubsection{Residual torque}

For low Earth orbit (LEO) spacecraft, the residual magnetic torque, $\mathbf{T}_{r s d}$, is usually the largest disturbance if the magnetic field of the electrical equipment is strong. The residual magnetic torque is computed as follows:

$$
\mathbf{T}_{r s d}=\mathbf{M}_{r s d} \times \mathbf{B}
$$

where $\mathbf{M}_{r s d} \in \mathbb{R}^{3}$ is the residual magnetic moment, which is assumed to be a vector of constant magnitude and a random direction. 


\subsection{CASSIOPE Spacecraft and Mission}

This section is dedicated to give a brief overview over the CASSIOPE spacecraft hardware configuration, mission and the available telemetry data with its frequency. This data is used for verifying the proposed work later in chapters 5 and 6 .

The CASSIOPE is a Canadian Space Agency (CSA) multi-mission satellite with the objectives of space weather information gathering and verifying high-speed communications concepts through the use of advanced space technologies [60, 84].

The telemetry data that is provided by the CASSIOPE technical team is for the CASSIOPE ground station tracking maneuvers. These telemetry data consists of the following:

1. Angular velocities' measurements

2. Magnetometer measurements

3. Dipole moments

4. Ephemeris:

(a) Attitude in terms of Euler angles where the rotation sequence is roll, pitch and yaw 
(b) Spacecraft Position ${ }^{i} \mathbf{R}$ and velocity ${ }^{i} \mathbf{V}$ in inertial frame

(c) Latitude, longitude and altitude in the World Geodetic System 1984 (WGS84) frame, and also in the Earth Centered Inertial (J2000) frame.

5. Torques from reaction wheels and magnetic rods

The above data are given with sampling frequency $10 \mathrm{~Hz}$ or $1 \mathrm{~Hz}$, except the ephemeris which are given with a sampling frequency of $0.2 \mathrm{~Hz}$. Linear interpolation is used for computing the ephemeris at sampling frequency $10 \mathrm{~Hz}$ or $1 \mathrm{~Hz}$. The duty cycle for the magnetic rods is $\delta=0.7$. The spacecraft angular velocities are given for the body frame w.r.t the inertial frame. The spacecraft inertia tensor matrix is as follows:

$$
[I]=\left[\begin{array}{ccc}
186.5202 & -0.6839 & -5.2728 \\
-0.6839 & 194.4095 & 4.2445 \\
-5.2728 & 4.2445 & 214.1428
\end{array}\right]\left[\mathrm{kg} \cdot \mathrm{m}^{2}\right]
$$

The given magnetic field measurements are in the body fame and they are the results from two magnetometers' measurements that are installed on two different booms. 


\section{Chapter 3}

\section{A New Efficient Variant of The}

\section{B-dot Control for Spacecraft}

\section{Magnetic Detumbling}

For small spacecraft, after separation from the launching mechanism, the spacecraft gained undesired angular momentum. This angular momentum derives the spacecraft to a tumbling process that leads to a chaotic uncontrolled undesired rotational motion. Therefore, a detumbling maneuver is required with a careful choice of attitude control system to reduce the risk of losing the control over the spacecraft. 
Magnetic detumbling using a B-dot control law does not need angular velocity measurements. The B-dot control law is a torque projection-based controller that utilizes the averaging method; however, it is not guaranteed to have the dipole moment orthogonal to the ambient magnetic field, especially as the angular velocity gets smaller during detumbling, resulting in a sub-optimal torque vector from the minimum residual torque perspective.

This chapter presents a new variant of the B-dot control law. By introducing a substitute of the angular velocity, based on the ambient magnetic field data, it is possible to develop a control law that guarantees the magnetic dipole moment to remain in the plane orthogonal to the ambient magnetic field. This new variant of Bdot law will preserve the Triple Orthogonality Condition (TOC) among the spacecraft applied torque, the generated dipole moment and the ambient magnetic field vector. This new variant is featured by its fast detumbling maneuver, low power consumption and even improve the system stability from stability theories perspective.

\subsection{Magnetic Detumbling Control Law}

In this section, a new modified B-dot control law is presented. A Lyapunov stability analysis is conducted for the dynamics of a rigid-body satellite actuated by the proposed B-dot control law and the one that proposed in [1]. Taking into account the 
intermittent actuation of the magnetic rods and magnetometer, unlike most of the existing studies in the literature.

\subsubsection{The Modified B-dot Control Law}

The simplest B-dot control law is the first early version introduced in 1976 in [26]. It was an elegant alternate solution of using the spacecraft angular velocity in the feedback loop. Avanzini and Giulietti [1] proposed a modified version of the B-dot law which is featured by its faster detumbling time. This control law is given by a static linear feedback of the form:

$$
\mathbf{M}=-\frac{k_{w}}{\|\mathbf{B}\|} \dot{\hat{\mathbf{B}}}
$$

where $k_{w}>0$ is a scalar control gain. The controller type here is a projection-based controller in which the goal is to find the projection of the required torque on the

plane that is perpendicular to the ambient magnetic field vector. Therefore, $\dot{\hat{\mathbf{B}}}$ is used instead of $\dot{\mathbf{B}}$. Many variants of the B-dot controller have been presented in the literature since then such as those in [27 31]. Avanzini and Giulietti proposed a form of $k_{w}$ gain in sub-optimal sense as follows [1] :

$$
k_{w}=2 \Omega\left(1+\sin \zeta_{m}\right) I_{\min }
$$


where $\zeta_{m}$ represents the inclination of the spacecraft orbit relative to the equatorial plane and $I_{\min }$ is the minimum inertia tensor component. The derivative of the measured ambient magnetic field vector could be computed using the transport theorem as follows [4, 82]:

$$
\dot{\mathbf{B}}=[R(\mathbf{q})]^{i} \dot{\mathbf{B}}+[\mathbf{B}]_{x} \boldsymbol{\omega}
$$

where $R(\mathbf{q})$ is computed using Eq. (2.2) and $\boldsymbol{\omega}$ is the spacecraft angular velocity of the $b$ frame with respect to the $i$ frame resolved in the $b$ frame (for notation simplification the post subscript for $\boldsymbol{\omega}$ is removed for this and the next entire chapters). The magnetic field variation is caused by four factors. The change in the magnetic field magnitude itself over time is the first factor, yet usually this change is very small and neglected. The second one is the rotation of the magnetic field itself around the Earth with $\overline{\boldsymbol{\omega}}$. The designers usually assume $\overline{\boldsymbol{\omega}}$ as an averaged constant value as seen in the inertial frame [4, 67]. The third factor is the spacecraft orbital motion $\Omega$, which causes the spacecraft to see a variation in the magnetic field at a rate equal to double the orbital rate $(2 \Omega)$, assuming perfect magnetic field symmetry in the simple dipole model [24, 39, 61, 63]. The last, and most dominant, cause is the angular velocity of the spacecraft $\boldsymbol{\omega}$, especially for tumbling spacecraft, where $\overline{\boldsymbol{\omega}} \ll 2 \Omega \ll \boldsymbol{\omega}$.

Therefore, the derivative of the ambient magnetic field vector $\dot{\mathbf{B}}$ could be considered 
as an indication to the spacecraft body angular velocity as shown in the second term on the right side of the Eq. 3.3$)$, through the matrix $[\mathbf{B}]_{x}$ which is a function of the measured ambient magnetic field itself, assuming that $\overline{\boldsymbol{\omega}} \ll 2 \Omega \ll \boldsymbol{\omega}$. The latter is an acceptable assumption and is usually used in stability analysis [4]. Therefore, with $\dot{\mathbf{B}} \approx \mathbf{B} \times \boldsymbol{\omega}$, the B-dot control law in Eq. 3.1 will be equivalent to the law proposed in [1], Eq. (3.4) :

$$
\mathbf{M}=-\frac{k_{w}}{\|\mathbf{B}\|}(\hat{\mathbf{B}} \times \boldsymbol{\omega})
$$

The first term, in the right side of Eq. (3.3), however, would have a non-negligible effect in case of low angular velocity, which typically occurs at the end of the detumbling maneuver. That is why the performance of the B-dot control law usually degrades at low angular velocities.

Here, in this study, the first term in Eq. (3.3), call it the residual term, will be included, presenting a new variant of the B-dot control law. The analysis presented here is based on the following assumptions:

Assumption 3.1 The derivative of the geomagnetic field in the inertial frame for the respective orbit at all time $t$ satisfies the following equation [4]:

$$
{ }^{i} \dot{\mathbf{B}}={ }^{i} \overline{\boldsymbol{\omega}} \times{ }^{i} \mathbf{B},
$$


As the magnetic field magnitude change has an insignificant effect on the time rate change of the magnetic field, therefore, it will be neglected here. By plugging Eq. (3.5) into Eq. (3.3), the derivative of the ambient magnetic field can be computed as follows:

$$
\dot{\mathbf{B}}=R(\mathbf{q})\left({ }^{i} \overline{\boldsymbol{\omega}} \times{ }^{i} \mathbf{B}\right)+\mathbf{B} \times \boldsymbol{\omega}=[\mathbf{B}]_{x} \tilde{\boldsymbol{\omega}}
$$

where $\tilde{\boldsymbol{\omega}}=\boldsymbol{\omega}-R(\mathbf{q})^{i} \overline{\boldsymbol{\omega}}$. The vector $\tilde{\boldsymbol{\omega}}$ can be computed from Eq. (3.6) using the singular robust inverse matrix by adding a small term, $[\chi] \tilde{\boldsymbol{\omega}}$, as follows:

$$
\dot{\mathbf{B}} \approx\left[[\mathbf{B}]_{x}+[\chi]\right] \tilde{\boldsymbol{\omega}} \approx[\Sigma] \tilde{\boldsymbol{\omega}}
$$

where $[\chi]$ is a diagonal matrix with positive elements, $0<\chi \ll 1$. For $\chi=0$, Eq. (3.7) is equivalent to Eq. (3.3). $[\Sigma]$ is positive definite and invertible matrix. Employing this property, $\tilde{\boldsymbol{\omega}}$ can be computed from Eq. (3.7) and used instead of $\boldsymbol{\omega}$ in Eq. (3.4) to arrive at the proposed variant of the B-dot control law:

$$
\mathbf{M}=-\frac{k_{w}}{\|\mathbf{B}\|}\left(\hat{\mathbf{B}} \times[\Sigma]^{-1} \dot{\hat{\mathbf{B}}}\right)
$$

The condition number of the $[\Sigma]$ matrix will be addressed later. 


\subsubsection{Lyapunov Analysis}

Most stability analysis conducted in the literature, such as in [1], neglect the effect of the residual term in Eq. (3.3) and the duty cycle $\delta$. In this section, these effects are introduced in the analysis to gain more insight into the proposed control and its effect on reducing the control effort required for the maneuver. Typically, the control objective is to lessen the spacecraft kinetic energy to zero in the ideal case, in which the equilibrium point is considered to be $\boldsymbol{\omega}=0$.

The effect of the intermittent actuation of the magnetic rods can be introduced by defining the following function:

$$
f\left(t, T_{s}, \delta\right)= \begin{cases}1 & 0 \leq \operatorname{Mod}\left(t, T_{s}\right) \leq \delta T_{s} \\ 0 & \delta T_{s} \leq \operatorname{Mod}\left(t, T_{s}\right) \leq T_{s}\end{cases}
$$

where $t$ is the time. The function $\operatorname{Mod}(x, y)$ is the modulo operation that finds the remainder after division of $x$ by $y$ (called the modulus of the operation). The function $f\left(t, T_{s}, \delta\right)=1$ during the activation of the magnetic rods and $f\left(t, T_{s}, \delta\right)=0$ during

the desaturation and magnetometer measurement period. The function $f\left(t, T_{s}, \delta\right)$ can be described in a compact form as follows: 


$$
f\left(t, T_{s}, \delta\right)=1+\frac{\operatorname{Mod}\left(\operatorname{Mod}\left(t, T_{s}\right), \delta T_{s}\right)-\operatorname{Mod}\left(t, T_{s}\right)}{\delta T_{s}}
$$

From here on, the arguments of $f$ will be dropped for simplification. The function $f$ is used to represent the intermittent actuation of the magnetic rods and magnetometers by multiplying $f$ and the dipole moment $\mathbf{M}$. For instance, consider the control law in Eq. (3.1) (also used in [1,) the dipole moment becomes:

$$
\mathbf{M}=-f k_{w} \frac{\dot{\hat{\mathbf{B}}}}{\|\mathbf{B}\|}
$$

The applied torque to the spacecraft, using Eqs (2.7), (3.11) and (3.3) will be:

$$
\mathbf{T}=-f k_{w}[\Gamma(t)] \boldsymbol{\omega}-f k_{w} \frac{[R(\mathbf{q})]^{i} \dot{\mathbf{B}} \times \mathbf{B}}{\|\mathbf{B}\|^{2}},
$$

where $[\Gamma(t)]=[\hat{\mathbf{B}}]_{x}[\hat{\mathbf{B}}]_{x}^{T}$ is a positive definite matrix [10, 42, [57].

The spacecraft kinetic energy will be used as a Lyapunov candidate function to check the stability of the system dynamics by using the control law in Eq. (3.11) as follows:

$$
V(\boldsymbol{\omega})=\frac{1}{2} \boldsymbol{\omega}^{T}[I] \boldsymbol{\omega},
$$

where the Lyapunov function $V(0)=0$ and $V(\boldsymbol{\omega})$ is positive definite elsewhere. The 
average theory will be employed in order to approximate the non-autonomous system with an autonomous one [85], under the following condition that is satisfied by the spacecraft orbit [10, 42, 57]:

$$
\left[\Gamma_{a v g}\right]=\lim _{T \rightarrow \infty} \frac{1}{T} \int_{0}^{T}[\hat{\mathbf{B}}]_{x}[\hat{\mathbf{B}}]_{x}^{T} d t>0
$$

Assumption 3.2 The gyroscopic coupling term $\boldsymbol{\omega} \times[I] \boldsymbol{\omega}$ will be neglected as it is usually small and being of second order term for $\|\boldsymbol{\omega}\| \ll 1$. Moreover, for almost every CubeSat configuration, The gyroscopic coupling term becomes zero [1].

An average for the the function $f$ can be computed over the pulse width to simplify the analysis, as follows:

$$
f_{\text {avg }}=\frac{1}{T_{s}} \int_{0}^{T_{s}} f\left(t, T_{s}, \delta\right) d t=\frac{1}{T_{s}}\left(\int_{0}^{\delta T_{s}} 1 d t+\int_{\delta T_{s}}^{T_{s}} 0 d t\right)=\delta
$$

The derivative of this Lyapunov candidate function, using Eqs. (2.5), (3.12), (3.14) and (3.15) will be as follows:

$$
\dot{V}(\boldsymbol{\omega})=-\delta k_{w} \boldsymbol{\omega}^{T}\left[\Gamma_{a v g}\right] \boldsymbol{\omega}-\delta k_{w} \boldsymbol{\omega}^{T} \frac{[R(\mathbf{q})]^{i} \dot{\mathbf{B}} \times \mathbf{B}}{\|\mathbf{B}\|^{2}}+\boldsymbol{\omega}^{T} \mathbf{T}_{D}
$$


Neglecting the disturbance torque $\mathbf{T}_{D}$, the derivative of the Lyapunov function $\dot{V}$ will not be guaranteed to be negative semi definite at all times, due to the presence of the second term. Note that if we neglect that term then $\dot{V}$ is negative semi definite, when we neglect $\mathbf{T}_{D}$. Specifically, it is the second term that may cause $\dot{V}$ to be positive. In such case, the previous conclusions in the literature that the system is stable in the sense of Lyapunov stability are not valid when accounting for the second term. In fact, this case occurs when the angular velocity is relatively small. As a result, most existing controls would converge from a high angular velocity state toward a value that is about twice the orbit rate, at which the control may push the system back away from that state, causing the angular velocity value to oscillate about the state of twice the orbit rate. Hence, this system is stable only in terms of Lagrange stability. Clearly, there is a wasted control effort in such case. Yet, at high angular velocities, the control effectively damps the motion. The same conclusion holds true for bounded disturbance torque.

Now, consider the proposed B-dot law in Eq. (3.8). The Euler Eq. (2.5) can be written in the inertial frame, along with using the torque Eq. (2.7), control law Eq. (3.8), the duty cycle effect through $f$, assumption 3.2, and ${ }^{i} \dot{\tilde{\omega}}={ }^{i} \dot{\boldsymbol{\omega}}$ (since $\overline{\boldsymbol{\omega}}$ is constant), as follows [4]:

$$
{ }^{i}[I] \stackrel{\dot{\tilde{\omega}}}{i}=-f k_{w}{ }^{i}[\Gamma(t)]^{i} \tilde{\boldsymbol{\omega}}+{ }^{i} \mathbf{T}_{D}
$$


The equilibrium point is considered at ${ }^{i} \tilde{\boldsymbol{\omega}}=0$. The spacecraft angular kinetic energy is used as a Lyapunov candidate function as follows:

$$
V 1\left({ }^{i} \tilde{\boldsymbol{\omega}}\right)=\frac{1}{2}{ }^{i} \tilde{\boldsymbol{\omega}}^{T i}[I]^{i} \tilde{\boldsymbol{\omega}}
$$

The Lyapunov candidate function $V 1(0)=0$ and $V 1\left({ }^{i} \tilde{\boldsymbol{\omega}}\right)$ is positive definite elsewhere. The time derivative of the Lyapunov function is:

$$
\dot{V} 1\left({ }^{i} \tilde{\boldsymbol{\omega}}\right)={ }^{i} \tilde{\boldsymbol{\omega}}^{T}{ }^{i}[I]^{i} \dot{\tilde{\boldsymbol{\omega}}}
$$

Using Euler Eq. (3.17), which is written in the inertial frame, the time derivative of the Lyapunov function is:

$$
\dot{V} 1\left({ }^{i} \tilde{\boldsymbol{\omega}}\right)=-f k_{w}{ }^{i} \tilde{\boldsymbol{\omega}}^{T}{ }^{i}[\Gamma]{ }^{i} \tilde{\boldsymbol{\omega}}+{ }^{i} \tilde{\boldsymbol{\omega}}^{T}{ }^{i} \mathbf{T}_{D}
$$

Ignoring the disturbance torque $\mathbf{T}_{D}$, the derivative of the Lyapunov function $\dot{V} 1$ is negative semi definite. Therefore, the system will be stable in the sense of Lyapunov stability. Moreover, asymptotic stability can be proved as follows. As the system is Lyapunov stable, the derivative $\dot{\tilde{\omega}}$ is non-zero. By continuous differentiation of $V 1$, 
the third derivative is takes the form:

$$
\dddot{V} 1\left(^{i} \tilde{\boldsymbol{\omega}}\right)=-2 \delta k_{w}{ }^{i} \dot{\tilde{\boldsymbol{\omega}}}^{T}\left[\Gamma_{a v g}\right]^{i} \dot{\tilde{\boldsymbol{\omega}}}-2 \delta k_{w}{ }^{i} \tilde{\boldsymbol{\omega}}^{T}\left[\Gamma_{a v g}\right] \stackrel{i}{i} \ddot{\tilde{\boldsymbol{\omega}}}
$$

When $\tilde{\boldsymbol{\omega}}=0$, the second term in the above equation vanishes, and hence the third derivative becomes negative definite. Hence the system is asymptomatically stable [82].

Remark 3.1: The actual system is not asymptotically stable as the function $f$ takes values of zero, at times, and hence there is no control authority during these times.

Remark 3.2: In the presence of a bounded disturbance torque, and as the spacecraft angular velocity becomes low, the disturbance torque in Eq. 3.20 could lead to increasing the angular velocity. However, the first term on the right hand side of Eq. (3.20) is negative semi definite, which means that once the angular velocity increases, the first term will dominate again and the angular velocity will be bounced back as the kinetic energy decays.

In summary, the main differences among the proposed B-dot law here and almost all the variants of the B-dot laws in the literature is that the current variants of the B-dot use control laws to drive the spacecraft angular velocity to zero with the following assumption $\overline{\boldsymbol{\omega}} \ll 2 \Omega \ll \boldsymbol{\omega}$. However, in our case, the goal of the control law is to drive the spacecraft angular velocity to converge to the value of $2 \Omega$. Furthermore, 
the proposed B-dot law preserves the TOC, unlike most of the existing B-dot laws by computing a substitute of the spacecraft angular velocity. These differences, as will be shown later in the next few sections, will improve the detumbling time and the required power consumption.

\subsubsection{The Proposed B-dot Law from Geometric Point of View}

A discussion is here presented for each of the control law in Eq. (3.4) (referred to as $\boldsymbol{\omega}$ law), the proposed form of the B-dot control law in Eq. (3.1) [1] (referred to as B-dot law), and the proposed variant of the B-dot control law in Eq. (3.8) (referred to as the TOC B-dot law).

Consider fig 3.1. For a specific spacecraft angular velocity vector $\boldsymbol{\omega}$, there is control torque, $\mathbf{T}_{r e q}$. This $\mathbf{T}_{r e q}$ vector can be written in terms of the spacecraft angular velocity as $\mathbf{T}_{r e q}=-k_{w} \boldsymbol{\omega}$. When using magnetic rods for control, the control torque is constrained to be perpendicular to the ambient magnetic field vector $\mathbf{B}$. The optimal projection of the $\mathbf{T}_{r e q}$ vector on the plane perpendicular to $\mathbf{B}$ is the vector $\mathbf{T}$, which is obtained by minimizing the residual torque vector $\Delta \mathbf{T}$, or equivalently

minimizing the angle between the $\mathbf{T}_{r e q}$ and $\mathbf{T}$ vectors. The optimality (the minimum residual torque $\Delta \mathbf{T}$ ) is fulfilled by computing the dipole moment vector $\mathbf{M}$ as a cross 


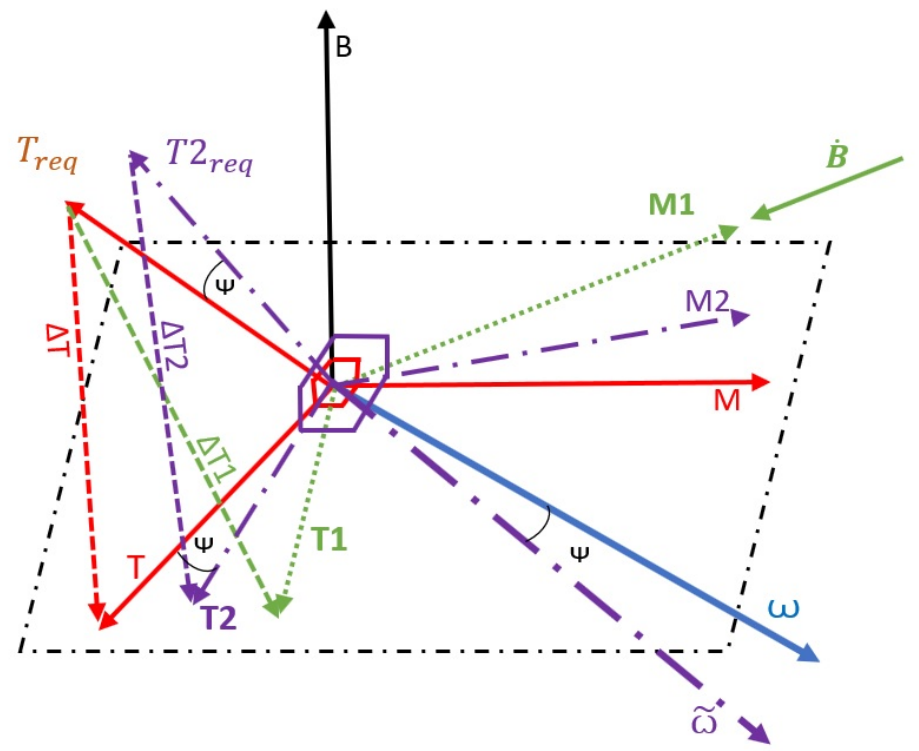

Figure 3.1: Desired torques and their projection for a given ambient magnetic field vector for the $\boldsymbol{\omega}$, B-dot and the TOC B-dot control laws.

product of the $\mathbf{T}_{r e q}$ and $\mathbf{B}$ vectors, which yields $\mathbf{M}$ in the plane orthogonal to $\mathbf{B}$, as can be seen in Eq. (3.4) and continuous red lines in fig 3.1 . Furthermore, the projected torque vector $\mathbf{T}$ (the applied torque) will be the optimal projection of the required torque $\mathbf{T}_{r e q}$. This ideal case assumes the availability of the spacecraft angular velocity $\boldsymbol{\omega}$ and the ambient magnetic field vector B. The $\boldsymbol{\omega}$ control law in Eq. (3.4) can be used in this case when the $\boldsymbol{\omega}$ and $\mathbf{B}$ are available.

The second control law, B-dot law, is based on the assumption that the spacecraft angular velocity in the detumbling mode is larger than double orbital rate $2 \Omega$, which is a realistic assumption. Therefore, the residual term in the ambient magnetic field derivative vector $\dot{\mathbf{B}}$ in Eq. (3.3) is neglected. Consequently, the required dipole moment vector M1, see green dotted lines in fig. 3.1 , is directly proportional to the vector 
$\dot{\mathbf{B}}$. This $\mathbf{M} 1$ vector is almost in the plane that is orthogonal to the $\mathbf{B}$ vector, for high spacecraft angular velocity. However, the generated torque $\mathbf{T} 1$ is not the optimal projection of the $\mathbf{T}_{r e q}$ corresponding to the true $\boldsymbol{\omega}$. As the spacecraft angular velocity decreases, the residual term effect in Eq. (3.3) increases, and leads to increasing the deviation of the $\mathbf{M} 1$ vector from the plane orthogonal to $\mathbf{B}$ or equivalently increases the angle between $\mathbf{M}$ and $\mathbf{M} 1$ vectors. Hence, the torque vector $\mathbf{T} 1$ will not be close to the optimal projection of the required torque from the minimum residual torque perspective. Hence the B-dot law is characterized by a good performance when the angular velocity is high; yet there is waisted power when the angular velocity is low. Moreover, the residual term in Eq. (3.3) will prevent such a controller to settle to zero angular velocity. There is always a steady state error that is about twice the orbital rate [39].

The TOC B-dot law, however, extracts information about the spacecraft substitute angular velocity $\tilde{\boldsymbol{\omega}}$ from the computed ambient magnetic field derivative vector $\dot{\mathbf{B}}$ using the magnetometer measurements. Then this $\tilde{\boldsymbol{\omega}}$ vector is used to find the required torque $\mathbf{T} \mathbf{2}_{\text {req }}$. In this case, the generated dipole moment will be in the plane that is orthogonal to the vector $\mathbf{B}$, see purple dashed lines in fig 3.1 . Hence, the TOC B-dot controller will have a similar principal of operation to that of the $\boldsymbol{\omega}$ law controller, without having to use angular velocity measurements.

Overall, the TOC B-dot law is characterized by its applicability for detumbling, for 
high angular velocity, without direct information about the spacecraft angular velocity, which is similar to the B-dot law and on the contrary to the $\boldsymbol{\omega}$ law. However, in the case of low angular velocity, the TOC B-dot law is characterized by energy saving in terms of the total magnetic rods' activation time; a behavior that is similar to the $\boldsymbol{\omega}$ control law and on the contrary to the B-dot law. Therefore, it results in a faster detumbling time in converging to the desired angular velocity with smaller magnetic rods' activation periods (less power consumption).

\subsection{Magnetometer Errors and Frequency Aliasing Considerations}

\section{Magnetometer Errors}

Some issues arise during actual implementation of the magnetic control system that should be accounted for in the simulation environment to get realistic results. Magnetometers usually suffer from constant and time-varying errors as discussed before in section 1.2.2 [53, [54, [59, 62]. Therefore, for low cost missions, magnetic field measurements are considered the weighted average of measurements collected during a measurement period, for one or more magnetometers, according to the following 
equation:

$$
\mathbf{B}=\sum_{k=1}^{y} w_{k}\left[R_{k}\right] \frac{\sum_{j=1}^{n} \mathbf{B}_{j}}{n}
$$

where $R_{k}$ is the transformation matrix from the sensor frame to the body frame, $w_{k}>0$ are weights satisfying $w_{1}+w_{2}+. .+w_{y}=1, y$ is the number of available magnetometers on board, and $n$ is the number of captured magnetometer measurements during the measurement period. This can be considered as a low pass filter.

Due to all the above mentioned errors, computing the derivative of the magnetic field B numerically becomes a challenge, and usually leads to inaccuracies and unnecessary higher power consumption by the rods. This error in computing the derivative numerically is proportional to $T_{s}^{x-1}$, where $T_{s}$ is the sampling period of the measurements and $x$ is the number of points used for computing the derivative [86]. Therefore, increasing the number of points used for computing the derivative reduces the error. For example, for $T_{s}=0.1[S e c]$ and five data points, the derivative error will be in the order of $\left(0.1^{4}\right)$. The formula for computing the magnetic field derivative at the current time $t$ using the five-point stencil method is given in Eq. 3.23).

$$
\dot{\mathbf{B}}(t) \approx \frac{3 \mathbf{B}\left(t-4 T_{s}\right)-16 \mathbf{B}\left(t-3 T_{s}\right)+36 \mathbf{B}\left(t-2 T_{s}\right)-48 \mathbf{B}\left(t-T_{s}\right)+25 \mathbf{B}(t)}{12 T_{s}}
$$




\section{Sensor Aliasing Considerations}

The spacecraft angular velocity is proportional to the ambient magnetic field derivative through the singular skew symmetric matrix that contains the information about the ambient magnetic field, as shown in Eq. (3.3). Therefore, the sampling time should be selected in such a way that the ambient magnetic field derivative provides acceptable measure for the spacecraft angular velocity. According to Nyquist criterion, the maximum frequency of changes in the ambient magnetic field that can be sensed without experiencing aliasing is $\frac{1}{2 T_{s}}[$ []. Therefore, the maximum rotational velocity that can be measured from two consecutive ambient magnetic field measurements is $\frac{\pi}{T_{s}}[\mathrm{rad} / \mathrm{s}]$, which leads to the following sampling time limitation rule $T_{s} \leq \frac{\pi}{\left|\boldsymbol{\omega}_{\max }\right|}$, where $\boldsymbol{\omega}_{\max }$ is the maximum expected angular velocity in any of the spacecraft axes [27]. From the stability point of view, the sampling time $T_{s}$ along with the duty cycle $\delta$ should be constrained through the relation [27]:

$$
T_{s} \leq \frac{\pi}{2 \delta\left|\boldsymbol{\omega}_{\max }\right|}
$$

Therefore, both constrains on the sampling time should be satisfied concurrently in order to avoid measurement aliasing, or torque application in the wrong direction. 


\subsection{Simulations and Discussions}

In this section, the performance of the TOC B-dot law is demonstrated via Monte Carlo simulations for comparison among the $\boldsymbol{\omega}$ control law, B-dot control law, and the TOC B-dot control law. For the $\boldsymbol{\omega}$ control law, a perfect knowledge of the spacecraft angular velocity is assumed available, regardless of the magnitude of the angular velocity. Therefore, the $\boldsymbol{\omega}$ law results are used as a reference for comparing the outputs from the B-dot law and the TOC B-dot law for the case study.

Data from the picosatellite Delfi-PQ project of Delft University of Technology is the used in the simulations [27]; these are listed in table 3.1 along with the orbital parameters. Fonod and Gill [27] presented a new variant of the B-dot control that saves $5 \%$ of the power while sacrificing only $0.5 \%$ of the time needed to detumble the satellite to the target angular velocity. At the end of this section, the results of the TOC B-dot control will be compared to the variable gain B-dot control presented in [27.

The earth magnetic field vector that computed from the WMM will be corrupted by the random noises and bias vectors. The rise and fall times are also considered during modeling of the magnetic rods. Disturbance torques are also modeled; table 3.2 presents the required parameters to compute these disturbance torques. Another 
Table 3.1

Spacecraft and orbital parameters

\begin{tabular}{lc}
\hline \hline Parameter & Value[unit], uncertainty $[1 \sigma]$ \\
\hline$\left[I_{x}, I_{y}, I_{z}\right]^{T}$ & {$[1.7311 .7260 .264]^{T}\left[\mathrm{~g} \cdot \mathrm{m}^{2}\right][20 \%]$} \\
Mass & $600[\mathrm{~g}][15 \%]$ \\
Max. Dipole Moment & $\pm 0.002\left[\mathrm{Am} \mathrm{m}^{2}\right][15 \%]$ \\
Rise/ fall times of magnetic rods & $0.01[\mathrm{~s}]$ \\
Magnetometers noise standard deviation & $600[\mathrm{nT}]$ \\
Magnitude of magnetometer bias vector & $400[\mathrm{nT}]$ \\
Inclination (Inc) & $96.85^{\circ}$ \\
Altitude (H) & $350[\mathrm{~km}]$ \\
Right Ascension of ascending node & $\pi / 4[\mathrm{rad}]$ \\
True anomaly & $\pi / 6[\mathrm{rad}]$ \\
Argument of perigee & $\pi / 2[\mathrm{rad}]$ \\
\hline
\end{tabular}

random direction torque with a magnitude of $\left\|T_{d}\right\|=2 \cdot 10^{-9}[N . m]$ is modeled to represent the unknown sources and modeling errors in the disturbance torque models and inertia uncertainty in the Euler equations, Eq. (2.5).

Table 3.2

Disturbance Parameters

\begin{tabular}{lc}
\hline \hline Parameter & Value[unit], uncertainty $[1 \sigma]$ \\
\hline$\rho^{*}$ & $2.01 .10^{-12}\left[\mathrm{Kg} \cdot \mathrm{m}^{3}\right]$ \\
Offset of center of pressure from center of mass & {$[4.52 .0-8.2][\mathrm{mm}][10 \%]$} \\
$C_{D}$ & 2.1 \\
Spacecraft cross section areas & {$[92.1122 .925 .2]\left[\mathrm{cm}^{2}\right]$} \\
Residual dipole moment vector magnitude & $1 e^{-4}\left[\mathrm{Am}^{2}\right][10 \%]$ \\
$c_{r k}$ & 1.5 \\
$F_{\text {solar }}$ & $1366\left[\mathrm{w} / \mathrm{m}^{2}\right]$ \\
Magnitude of disturbance torque $\left\|T_{d}\right\|$ & $2.10^{-9}[\mathrm{~N} . \mathrm{m}]$ \\
\hline${ }^{*} \rho$ is computed by empirical formula in http://www.braeunig.us/space/atmmodel.htm $^{2}$
\end{tabular}

The parameters of the control algorithms are as follows: the duty cycle percentage is $\delta=0.6$, assuming the maximum expected angular velocity in each axis is less than $1.5 \pi[\mathrm{rad} / \mathrm{sec}]$. The sampling time $T_{s}$ according to the Nyquist criteria should 
be $T_{s} \leq 0.55[\mathrm{sec}]$ to avoid sensor aliasing as discussed in section 3.2 . Here in this study, it is selected as $T_{s}=0.25[\mathrm{sec}]$. The gain $k_{w}$ for the three controllers is computed using Eq. (3.2). The detumbling time $t_{\text {det }}$ is computed here by adding a 10-minute confirmation window after the spacecraft reaches the desired angular velocity $\boldsymbol{\omega}_{\text {des }}=0.5[\mathrm{deg} / \mathrm{s}]$.

\section{Case Study}

Before presenting the statistical Monte Carlo analysis, the results from a sample example run for the TOC B-dot law are presented. In this example, the satellite has initial angular velocity selected randomly. Figure 3.2 depicts the time behavior of the satellite's angular velocity. It can be seen that the proposed algorithm is able to detumble the spacecraft from high initial angular velocity. After reaching the required target angular velocity $\boldsymbol{\omega}_{\text {des }}=0.5[\mathrm{deg} / \mathrm{s}]$, the TOC B-dot law is still active and preserving the spacecraft angular velocity within the required desired rate. In this case study, the derivative of the magnetic field $\dot{\mathbf{B}}$ is computed using the five-stencil method, see Eq. (3.23) and $\chi=10^{-6}$ is selected.

In order to emphasis the impact of numerical computation of the magnetic field

derivative $\dot{\mathbf{B}}$, a comparison for the absolute angler velocities history is conducted, see fig. 3.3, for the $\boldsymbol{\omega}$ control law labeled ( $\boldsymbol{\omega}$ law), B-dot law Eq. (3.1) labeled (B-dot), 

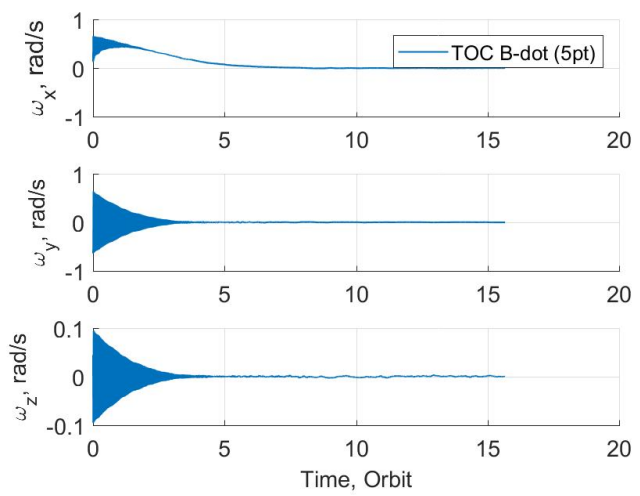

Figure 3.2: Angular velocity history w.r.t inertial frame

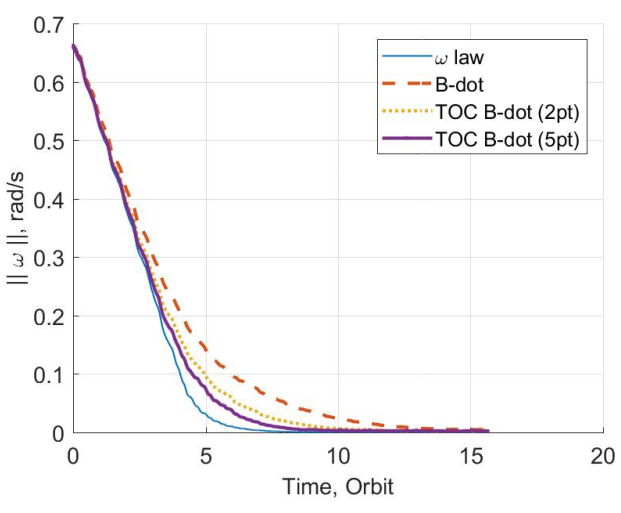

Figure 3.3: Absolute angular velocity Comparison

TOC B-dot control law that preserves TOC with backward difference scheme labeled (TOC B-dot (2pt)) and TOC B-dot control law with five-stencil method (TOC Bdot (5pt)). As shown in fig 3.3, the TOC B-dot law, for both methods of numerical computation of the derivative, is faster than the B-dot law in Eq. (3.1). However, the performance of proposed law with the five-stencil method is better.

The above sample simulation is repeated for the three controllers and for several $\chi$ values for the TOC B-dot law. The results for the $\boldsymbol{\omega}$ law controller is the reference and is used for normalizing the results from the other two controllers, B-dot and the TOC B-dot. During this section, two parameters are used for comparison; the mean value of the total activation time of the three magnetic rods $\mathbf{t}_{\text {on }}$, computed using Eq. (1.1), as an indication of the power consumption, and the converge time or the detumbling time $t_{d e t}$ to bring the spacecraft angular velocity down to the $\boldsymbol{\omega}_{\text {des }}$.

Figure 3.4 depicts the normalized $t_{\text {det }}$ for the B-dot and the TOC B-dot control. As 


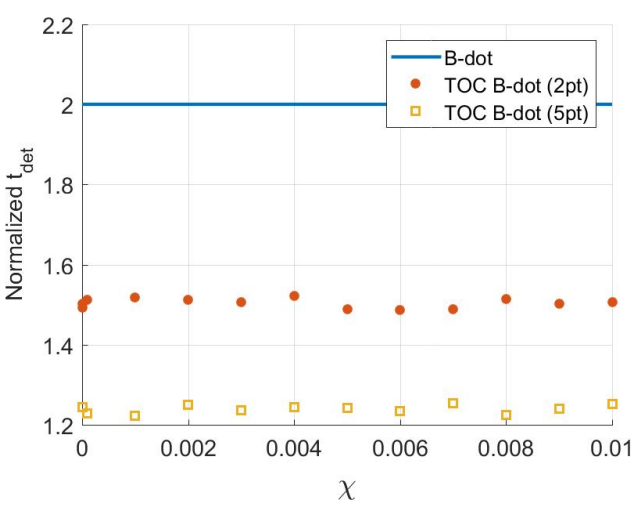

Figure 3.4: TOC B-dot $t_{\text {det }}$ versus $\chi$ change

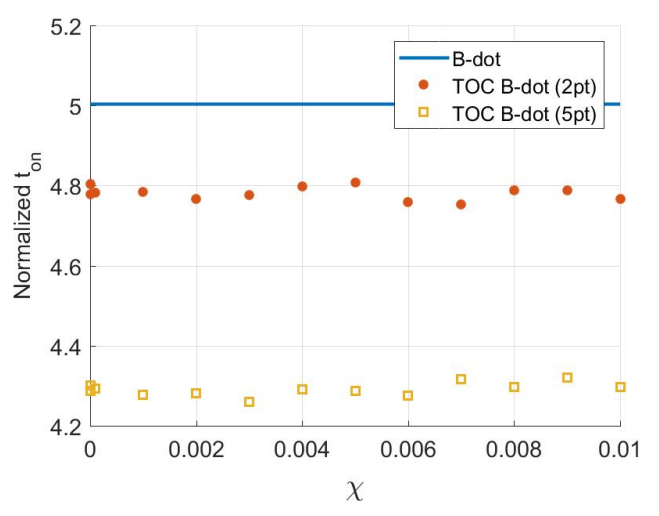

Figure 3.5: TOC B-dot $\mathbf{t}_{\text {on }}$ versus $\chi$ change

expected the TOC B-dot performance with the case of computing the derivative using the five-stencil method is better with $\chi$ change.

As a measure for the consumed power by the magnetic rods, fig 3.5 depicts the mean of the normalized values of the magnetic rods activation time $\mathbf{t}_{o n}$, for the B-dot and the TOC B-dot algorithms normalized with respect to the results from the $\boldsymbol{\omega}$ control law. Same results are obtained as in fig. 3.4.

As can be observed from the previous results, the TOC B-dot controller gives faster convergence time compared to the B-dot controller, and requires less power than the B-dot controller. These results are further confirmed in the Monte Carlo simulation section below. As shown in figs 3.4 and 3.5 , there is a wide range of possible values of $\chi$. Therefore, there is no tight requirement on the arithmetic precision of the onboard controller, from the $\chi$ prospective. Moreover, as can be seen from figs.3.4 and 3.5, the $\chi$ can take values up to 0.01 without much degradation in the performance. 
Hence, there is no condition number issue for the $[\Sigma]$ matrix. In the next Monte Carlo analysis, the $\chi$ will be selected as $1 \times 10^{-6}$ and magnetic field derivative will be computed using the five-stencil method.

\section{Monte Carlo simulation analysis}

Results are here presented for 4,000 Monte Carlo simulation runs, 2,000 for each of the B-dot and the TOC B-dot control laws. For each run, different noise seeds are utilized assuming Gaussian distribution. The results from the B-dot law Eq. (3.1) is used for normalization the results for the TOC B-dot law. In order to investigate the impact of the initial angular rate, each 2,000 runs is categorized into 400 categories. Each category has a different initial angular rate. Specifically, 200 categories

have their initial values constrained to be in the range $0.1 \pi[\mathrm{rad} / \mathrm{s}]$ and $-0.1 \pi[\mathrm{rad} / \mathrm{s}]$ (small initial angular velocity), While the other 200 categories have their initial angular rates constrained to be in the range of $1.5 \pi[\mathrm{rad} / \mathrm{s}]$ and $-1.5 \pi[\mathrm{rad} / \mathrm{s}]$ (high initial angular velocity). So, each selection of the initial angular rate is simulated 5 times, and the results of the activation time and detumbling time are averaged. The selected categories span ranges for high angular velocities and low angular velocities, to highlight the significance of the TOC B-dot controller, compared to the B-dot controller. Specifically, the latter is designed to work best in the case of high angular velocities. 


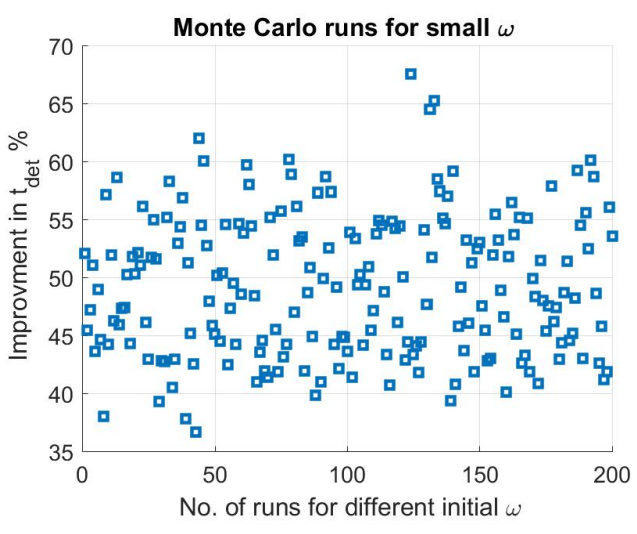

Figure 3.6: Improvement percentage in $t_{\text {det }}$ for the TOC B-dot at $\chi=1 e^{-6}$ for 200 different initial small $\boldsymbol{\omega}$.

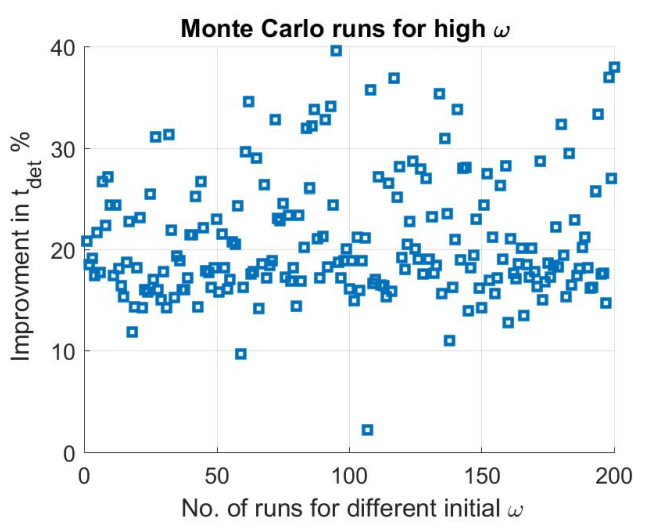

Figure 3.7: Improvement percentage in $t_{\text {det }}$ for the TOC B-dot at $\chi=1 e^{-6}$ for 200 different initial high $\boldsymbol{\omega}$.

The results of the simulation runs are here discussed. Figure 3.6 depicts the improvement percentage in the detumbling time $t_{d e t}$ for the TOC B-dot control law, compared to $t_{d e t}$ of the B-dot control, for the 200 categories of small initial angular velocities (2,000 Monte Carlo runs). Figure 3.6 shows that the detumbling time of the TOC B-dot control law is significantly less than that of the B-dot control. For the categories of high initial angular velocity, fig. 3.7 depicts the improvement percentage in the detumbling time $t_{d e t}$ for the TOC B-dot control law for another 2,000 Mont Carlo runs for the 200 high initial angular velocities. Figure 3.7 shows that the detumbling time for the TOC B-dot control law is still less than the detumbling time for the B-dot control law. This is expected for high angular velocities.

For further assessment, a histogram and a Gaussian fit analysis is conducted. Figure 3.8 shows the histogram and the Gaussian fit for the results presented in fig. 3.6 , in terms of the reduction percentage in the detumbling time when using the TOC B-dot 


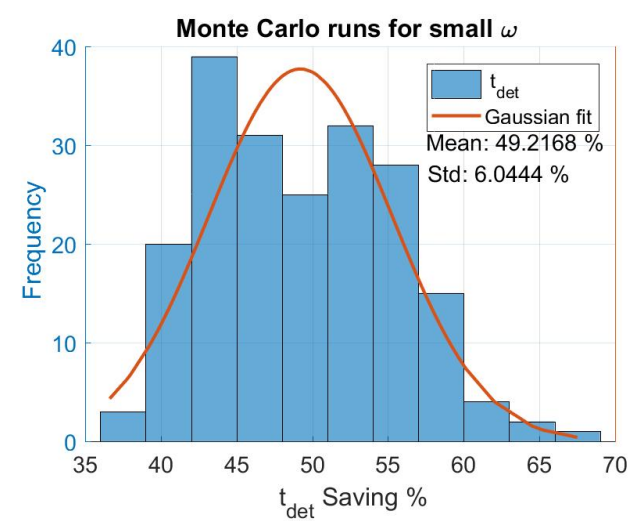

Figure 3.8: $t_{\text {det }}$ reduction percentage of $t_{\text {det }}$ for 200 different initial small $\boldsymbol{\omega}$. Histogram represents results of fig. 3.6 .

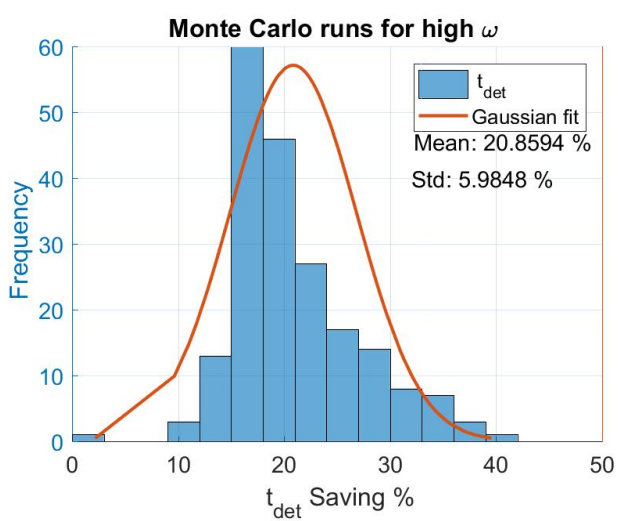

Figure 3.9: $t_{\text {det }}$ reduction percentage of $t_{d e t}$ for 200 different initial high $\boldsymbol{\omega}$. Histogram represents results of fig. 3.7 .

control law compared to the B-dot control law. As can be seen from fig 3.8, the mean value of the percent saving in terms of the detumbling time is $49.2 \%$, with $6.04 \%$ as standard deviation, for small initial angular rates. While, fig. 3.9 shows the histogram and the Gaussian fit for the results in fig 3.7 in terms of the reduction percentage in the detumbling time. A mean value of $20.86 \%$ reduction in the detumbling time is achieved, with $5.98 \%$ as standard deviation for high initial angular velocities.

The second comparison parameter is the activation time for the magnetic rods $\mathbf{t}_{\text {on }}$, which is considered a measure for the power consumption. Figure 3.10 depicts the improvement percentage of the mean of the total activation time $\mathbf{t}_{\text {on }}$ of the magnetic rods for the TOC B-dot control law w.r.t the results from the B-dot control law for the 200 categories of small initial angular velocities (2,000 Monte Carlo runs). Figure 3.10 shows that the normalized mean of the total activation time is reduced by the TOC B-dot control law compared to the B-dot control law. For high initial angular 


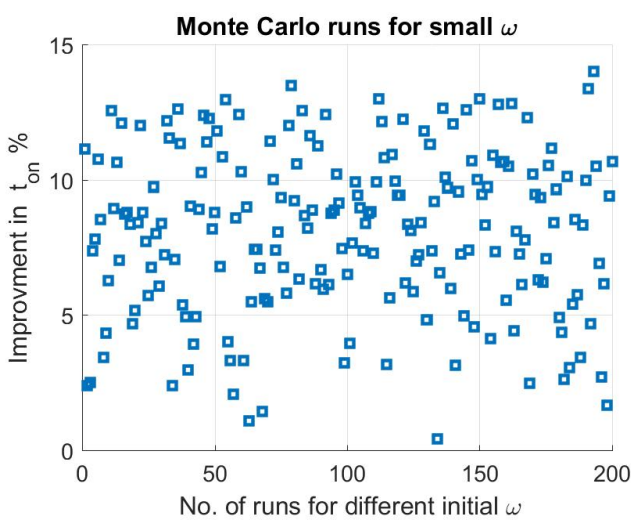

Figure 3.10: Improvement percentage in $\mathbf{t}_{\text {on }}$ for the TOC B-dot at $\chi=1 e^{-6}$ for 200 different initial small $\boldsymbol{\omega}$.

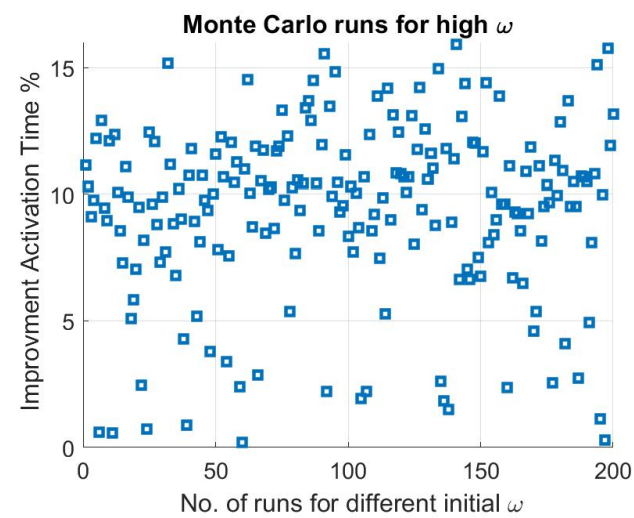

Figure 3.11: Improvement percentage in $\mathbf{t}_{\text {on }}$ for the TOC B-dot at $\chi=1 e^{-6}$ for 200 different initial high $\boldsymbol{\omega}$.

velocities, fig. 3.11 depicts the improvement percentage of the mean of the total activation time $\mathbf{t}_{\text {on }}$ of the magnetic rods for the other 2,000 Monte Carlo runs of high initial angular velocities. Figure 3.11 shows the mean of the total activation time of the magnetic rods for the TOC B-dot control law is still less than the mean of the total activation time of the magnetic rods for the B-dot control law.

Figure 3.12 shows the histogram and the Gaussian fit for the results in fig 3.10 in terms of the power saving percentage when using the TOC B-dot control law compared to the B-dot control law. The mean value for the power saving is $8.097 \%$, with a $3.00 \%$ standard deviation, when using the TOC B-dot controller compared to the B-dot control law, for small initial angular velocities. For the cases of high angular velocities, fig. 3.13 shows the histogram and the Gaussian fit for the results in fig. 3.11 in terms of the power saving percentage. The mean value for the power saving is $9.38 \%$, with $3.4 \%$ as standard deviation. 


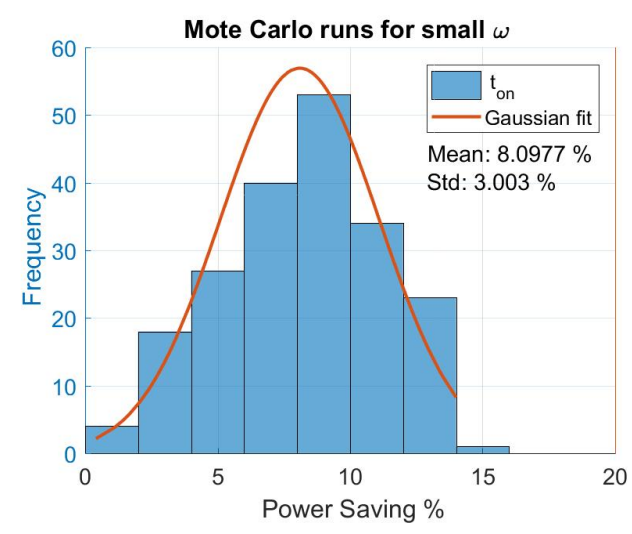

Figure 3.12: Power saving percentage for 200 different initial small $\boldsymbol{\omega}$. Histogram represents results of fig. 3.10 .

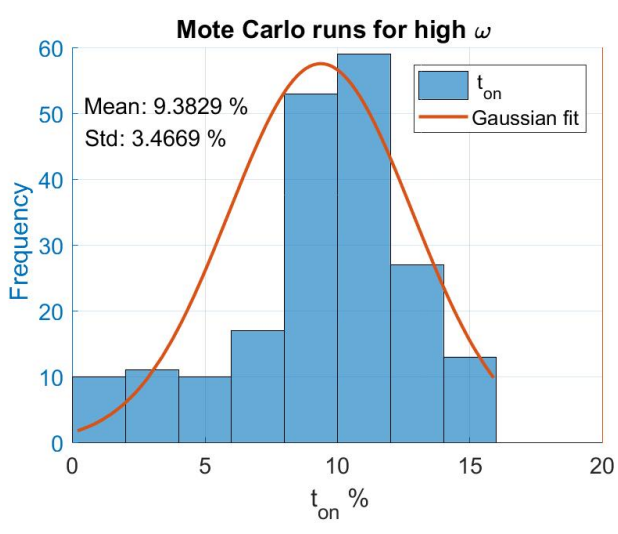

Figure 3.13: Power saving percentage for 200 different initial high $\boldsymbol{\omega}$. Histogram represents results of fig. 3.11 .

To investigate in more detail the power consumption savings, another case study is considered here. The average sum of the activation times $\mathbf{t}_{\text {on }}$ per orbit is calculated, for each magnetic rod axis; these are plotted in fig 3.14 for the two considered B-dot laws, for very high initial angular velocity. A reduction in average power consumption can be observed (especially at the end of the detumbling,) when using the TOC Bdot controller. The lower sub-plot represents the mean activation time of the three magnetic rods. The activation time on the vertical axis is normalized by orbit period. The total activation time when using the TOC B-dot control law is less than the total activation time when using the B-dot control law; the savings is about $8 \%$ for this case.

Finally, the TOC B-dot control is here compared to one of the most recent developments in the literature, Ref. [27]. Reference [27] presents a variant of the B-dot control, in which the control gain is variable, with the goal of achieving less power 


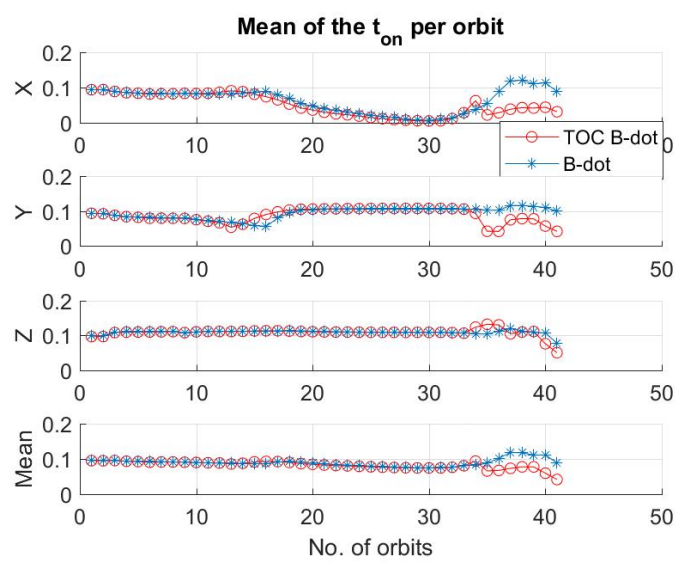

Figure 3.14: Magnetic rods $\mathbf{t}_{\text {on }}$ for B-dot and TOC B-dot control laws for the $\mathrm{X}, \mathrm{Y}$, and $\mathrm{Z}$ axes and the mean in orbit period scale.

consumption (Note that the TOC B-dot control preserves a constant control gain, which is computed using Eq. (3.2)). To conduct this comparison, a Mont Carlo simulation is conducted consisting of 50 runs. All runs use the same data provided in Ref. [27]. The initial angular velocity used in Ref. [27] is $180[\mathrm{deg} / \mathrm{s}]$ along each axis, which is also used in these simulations using the TOC B-dot control. The same two parameters of assessment used above are also used here for comparison; namely the magnetic rod activation time (a measure for power consumption) and the detumbling time are used for comparison.

The results of the Monte Carlo simulations using the TOC B-dot control are presented in table 3.3 . Table 3.3 also lists the results of using a variable control gain as reported in Ref. [27]. As shown in table 3.3, the TOC B-dot control demonstrates less power consumption by the magnetic rods, compared to the control in Ref. [27]. Specifically, the fourth raw shows a $13.29 \%$ savings of the TOC B-dot control in terms of 
power consumption compared to the B-dot control, whereas the variable gain control achieves a saving of $4.88 \%$ compared to the B-dot control. The power saving along each axis of the magnetic rods are listed in the first three rows in table 3.3 . The last row in the table 3.3 shows the detumbling time. The detumbling time of the TOC B-dot control is $14.03 \%$ less than that of the B-dot control, whereas the detumbling time of the variable gain control in Ref. [27] is $0.45 \%$ higher than the B-dot control. In summary, comparing the TOC B-dot control to the variable gain control in Ref. [27], the former has better power consumption, and better detumbling time.

Table 3.3

Efficiency in detumbling and activation times percentage

\begin{tabular}{lcc}
\hline Parameter & TOC B-dot & B-dot in [27] \\
& $\eta$ & $\eta$ \\
\hline$\left(\mathbf{t}_{\text {on }}\right)_{x}$ & $-18.81 \%$ & $-6.32 \%$ \\
$\left(\mathbf{t}_{\text {on }}\right)_{y}$ & $-10.96 \%$ & $-5.43 \%$ \\
$\left(\mathbf{t}_{\text {on }}\right)_{z}$ & $-11.83 \%$ & $-3.05 \%$ \\
$\sum \mathbf{t}_{\text {on }}$ & $-13.29 \%$ & $-4.88 \%$ \\
$t_{\text {det }}$ & $-14.03 \%$ & $0.45 \%$ \\
\hline
\end{tabular}

\subsection{Conclusion}

A new variant of the B-dot control is presented here. The TOC B-dot control maintains the magnetic dipole moment vector in the plane perpendicular to the ambient magnetic field, a condition that most of the B-dot control algorithms do not guarantee. Classically, the control algorithms that guarantee this condition require the measurement of the spacecraft angular velocity. The TOC B-dot control has the 
advantage of satisfying this condition without measuring the angular velocity. The TOC B-dot control is compared to the one of the fastest variant of B-dot control in the literature, a control law that assumes the availability of the angular velocity measurements, and a recent variable gain B-dot control. Monte Carlo simulations are conducted to carry out these comparisons. The results of the numerical simulations demonstrate that the TOC B-dot control achieves faster detumbling. with less power consumption power compared to other two B-dot control logics. 



\section{Chapter 4}

\section{Time-Optimal Magnetic Attitude}

\section{Detumbling}

The problem of minimum-time spacecraft attitude detumbling using magnetic rods is revisited in this chapter within the context of optimal control theory. Two formulations are presented; the first one assumes the availability of the angular velocity and the ambient magnetic field measurements for feedback. The second formulation assumes the availability of only the ambient magnetic field measurements in the feedback. In both formulations, the constraint in this optimal control problem is a limit on the maximum magnetic dipole moment of the magnetic rods.

It is shown that the time optimality will be achieved if the Triple Orthogonality 
Condition (TOC) among the torque, dipole moment and magnetic field vectors is fulfilled. This triple orthogonality is considered as a condition of optimality, which is often neglected in the existing B-dot law and its variants in the literature. The Pontryagin Minimum Principle is used to derive the control logic, for each formulation, in this non-autonomous system. The second formulation is shown to yield a new variant of the B-dot law.

\subsection{Time-Optimal Control}

The time optimal control problem is to find the optimal control $\mathbf{M}^{*}(t)$ which causes the system dynamics (Eq. (2.5)) to give the trajectory $\boldsymbol{\omega}^{*}(t)$ that minimizes the detumbling time, where $\left(.{ }^{*}\right)$ indicates the optimal value. This problem is of the type of Lagrange problems. The final time $t_{f}$ is free, the initial state variable $\boldsymbol{\omega}(0)=\boldsymbol{\omega}_{o}$, and the final state variable $\boldsymbol{\omega}\left(t_{f}\right)=0$. The cost function can be written as:

$$
J=\int_{t_{o}}^{t_{f}} 1 d t=t_{f}-t_{o}=t^{*}
$$

The control input satisfies the constraint in Eq.44.2). 


$$
\mathbf{M}_{i}(t) \leq \bar{M}_{\max }
$$

where $i=x, y, z$ and $\bar{M}_{\max }$ is the maximum allowable dipole moment magnitude for each rod.

\subsubsection{Case 1: Using Angular Velocity Measurements}

In this section, it is assumed that spacecraft angular velocities are available along with the measurements of the spacecraft ambient magnetic field. The solution of the optimal control problem of constrained control command is here solved using the Pontryagin Minimum Principle (PMP). The Hamiltonian $H \in \mathbb{R}^{1}$ of the system can be written using Eq. (2.5) and (4.1) as follows [87, 88]:

$$
H(\boldsymbol{\omega}(t), \boldsymbol{\lambda}(t), \mathbf{M}(t))=1-\boldsymbol{\lambda}(t)^{T}[I]^{-1}(\boldsymbol{\omega}(t) \times[I] \boldsymbol{\omega}(t))+\boldsymbol{\lambda}(t)^{T}[I]^{-1}[\mathbf{B}(t)]_{x}^{T} \mathbf{M}(t),
$$

where $\boldsymbol{\lambda} \in \mathbb{R}^{3}$ is the co-state variable, or Lagrange multiplier, and $(.)^{T}$ is the transpose of a matrix or vector. From here on, the arguments will be dropped for simplification in this section. 
The optimal control condition using the PMP must satisfy the following [87, 88]

$$
H\left(\boldsymbol{\omega}^{*}, \boldsymbol{\lambda}^{*}, \mathbf{M}^{*}\right) \equiv \min _{\mathbf{M}_{i}(t) \leq \bar{M}_{\max }}\left\{H\left(\boldsymbol{\omega}^{*}, \boldsymbol{\lambda}^{*}, \mathbf{M}\right)\right\} \leq H\left(\boldsymbol{\omega}^{*}, \boldsymbol{\lambda}^{*}, \mathbf{M}\right)
$$

By plugging Eq. 4.3 into Eq. (4.4), the following condition is obtained

$$
\boldsymbol{\beta}^{*} \mathbf{M}^{*}=\min _{\mathbf{M}_{i}(t) \leq \bar{M}_{\max }}\left\{\boldsymbol{\beta}^{*} \mathbf{M}\right\}
$$

where $\boldsymbol{\beta}^{*} \in \mathbb{R}^{1 x 3}$ is a row vector and is defined as follows:

$$
\boldsymbol{\beta}^{*}=\boldsymbol{\lambda}^{* T}[I]^{-1}[\mathbf{B}]_{x}^{T}
$$

To find the optimal solution $\mathbf{M}^{*}$, consider that:

$$
\min _{\mathbf{M}_{i}(t) \leq \bar{M}_{\text {max }}}\left\{\boldsymbol{\beta}^{*} \mathbf{M}\right\}=\min _{\mathbf{M}_{i}(t) \leq \bar{M}_{\text {max }}}\left\{\boldsymbol{\beta}_{x}^{*} \mathbf{M}_{x}+\boldsymbol{\beta}_{y}^{*} \mathbf{M}_{y}+\boldsymbol{\beta}_{z}^{*} \mathbf{M}_{z}\right\}
$$

where the subscript $i$ indicates the $i^{t h}$ component of a vector, $\forall i=x, y, z$. For each component in the above equation to be a minimum, the value of $\mathbf{M}_{i}$ must have the opposite sign of $\beta_{i}, \forall i=x, y, z$. Moreover, the magnitude of the vector $\mathbf{M}$ should take its maximum value to guarantee that Eq. (4.5) is satisfied. Hence Eq. 4.5 can 
be satisfied if $\mathbf{M}^{*}$ is selected to be in the opposite direction of $\boldsymbol{\beta}^{*}$, with a magnitude equal to its maximum value; that is:

$$
\mathbf{M}^{*}=-\frac{\boldsymbol{\beta}^{* T}}{\left\|\boldsymbol{\beta}^{* T}\right\|} M_{\max }
$$

where $M_{\max }=\frac{\bar{M}_{\max }}{\max \left|\hat{\beta}^{*}\right|}$ to ensure the intrinsic limits for each magnetic rods Eq. (4.2), where $|\cdot|$ is the absolute value, $\|\cdot\|$ represents the Euclidean norm of a vector and $\bar{M}_{\max } \leq\left\|\mathbf{M}^{*}\right\| \leq \sqrt{3} \bar{M}_{\max }$. The necessary conditions for optimality are:

1. The Lagrange multiplier $\boldsymbol{\lambda}$ must satisfy:

$$
\dot{\boldsymbol{\lambda}}=-\frac{\partial H}{\partial \boldsymbol{\omega}}=\left([I \boldsymbol{\omega}]_{x}-[I][\boldsymbol{\omega}]_{x}\right)[I]^{-1} \boldsymbol{\lambda}
$$

2. The states' variables must satisfy the following condition:

$$
\dot{\boldsymbol{\omega}}=\frac{\partial H}{\partial \boldsymbol{\lambda}}=-[I]^{-1}(\boldsymbol{\omega} \times[I] \boldsymbol{\omega})-[I]^{-1}[B]_{x}^{T} \frac{B \times[I]^{-1} \boldsymbol{\lambda}}{\left\|B \times[I]^{-1} \boldsymbol{\lambda}\right\|} M_{\max }
$$

The optimal control law in Eq. (4.8) indicates that the control law is a function of the optimal co-state variable $\boldsymbol{\lambda}^{*}$. Hence, Eq. (4.9) and (4.10) must be solved to determine the optimal co-state variable $\boldsymbol{\lambda}^{*}$. In general, Solving Eqs. 4.9 and 4.10 is a twopoint boundary value problem for this nonlinear non-autonomous system. This is a difficult problem to solve analytically. Consider, however, the following proposition: 
Proposition 4.1: The co-state definition given in Eq. 4.11 satisfies the necessary conditions of optimality given in Eq. (4.9) and Eq. (4.10).

$$
\boldsymbol{\lambda}^{*}=\frac{[I]^{2} \boldsymbol{\omega}^{*}}{\left\|[I] \boldsymbol{\omega}^{*}\right\| M_{\max }}
$$

Proof: By plugging Eq. 4.11) into the control law in Eq. 4.8, we obtain a closed loop form of the optimal control:

$$
\mathbf{M}^{*}=-\frac{B \times[I] \boldsymbol{\omega}^{*}}{\left\|B \times[I] \boldsymbol{\omega}^{*}\right\|} M_{\max }
$$

The optimal trajectory of the state variable $\boldsymbol{\omega}^{*}$ can be obtained by plugging Eq. 4.11 into Eq. 4.10 to get:

$$
\dot{\boldsymbol{\omega}}^{*}=-[I]^{-1}\left(\boldsymbol{\omega}^{*} \times[I] \boldsymbol{\omega}^{*}\right)-[I]^{-1}[B]_{x}^{T} \frac{B \times[I] \boldsymbol{\omega}^{*}}{\left\|B \times[I] \boldsymbol{\omega}^{*}\right\|} M_{\max }
$$

Differentiating Eq. 4.11, we get:

$$
\dot{\lambda}^{*}=\frac{\overbrace{[I]^{2} \dot{\boldsymbol{\omega}}^{*}\left\|[I] \boldsymbol{\omega}^{*}\right\| M_{\max }}^{\left(\left\|[I] \boldsymbol{\omega}^{*}\right\| M_{\max }\right)^{2}}}{\mathbf{W}_{1}}-\overbrace{\frac{[I]^{2} \boldsymbol{\omega}^{*} \boldsymbol{\omega}^{* T}[I]^{2} \dot{\boldsymbol{\omega}}^{*} M_{\max }}{\left\|[I] \boldsymbol{\omega}^{*}\right\|^{3} M_{\max }^{2}}}^{\mathbf{W}_{2}}
$$


Substituting for $\dot{\boldsymbol{\omega}}^{*}$ in the term $\mathbf{W}_{2}$ from Eq. (4.13), we get:

$$
\mathbf{W}_{2}=-\frac{[I]^{2} \boldsymbol{\omega}^{*} \boldsymbol{\omega}^{* T}[I]^{2}\left([I]^{-1}\left(\boldsymbol{\omega}^{*} \times[I] \boldsymbol{\omega}^{*}\right)\right)}{\left\|[I] \boldsymbol{\omega}^{*}\right\|^{3} M_{\max }}-\frac{[\Gamma]^{T}[I]^{2} \boldsymbol{\omega}^{*} \boldsymbol{\omega}^{* T}[I]^{2} \boldsymbol{\omega}^{*}}{\left\|[I] \boldsymbol{\omega}^{*}\right\|^{3}\left\|B \times[I] \boldsymbol{\omega}^{*}\right\|}
$$

where $[\Gamma]=[\mathbf{B}]_{x}[\mathbf{B}]_{x}^{T}$ and $\boldsymbol{\omega}^{*} \boldsymbol{\omega}^{* T} \in \mathbb{R}^{3 x 3}$ is a symmetric matrix. Consider the first term in Eq.4.15):

$$
\left.[I]^{2} \boldsymbol{\omega}^{*} \boldsymbol{\omega}^{* T}[I]^{2}\left([I]^{-1}\left(\boldsymbol{\omega}^{*} \times[I] \boldsymbol{\omega}^{*}\right)\right)=[I]^{2} \boldsymbol{\omega}^{*}\left([I] \boldsymbol{\omega}^{*}\right)^{T}\left(\boldsymbol{\omega}^{*} \times[I] \boldsymbol{\omega}^{*}\right)\right)=0
$$

Also, note that:

$$
\boldsymbol{\omega}^{* T}[I]^{2} \boldsymbol{\omega}^{*}=\left\|[I] \boldsymbol{\omega}^{*}\right\|^{2}
$$

Then the term $\mathbf{W}_{2}$ reduces to:

$$
\mathbf{W}_{2}=-\frac{[\Gamma]^{T}[I]^{2} \boldsymbol{\omega}^{*}}{\left\|[I] \boldsymbol{\omega}^{*}\right\|\left\|B \times[I] \boldsymbol{\omega}^{*}\right\|}
$$

Substituting for $\dot{\boldsymbol{\omega}}^{*}$ in the term $\mathbf{W}_{1}$ from Eq. (4.13), we get:

$$
\mathbf{W}_{1}=-\frac{[I]^{-1}\left(\boldsymbol{\omega}^{*} \times[I] \boldsymbol{\omega}^{*}\right)}{\left\|[I] \boldsymbol{\omega}^{*}\right\| M_{\max }}-\frac{[\Gamma]^{T}[I]^{2} \boldsymbol{\omega}^{*}}{\left\|[I] \boldsymbol{\omega}^{*}\right\|\left\|B \times[I] \boldsymbol{\omega}^{*}\right\|}
$$

Substituting the terms $\mathbf{W}_{1}$ and $\mathbf{W}_{2}$ from Eq. 4.19) and (4.18), respectively, into Eq. (4.14), The derivative of the co-state variable $\boldsymbol{\lambda}^{*}$ reduces to: 


$$
\dot{\lambda}^{*}=-\frac{[I]^{-1}\left(\boldsymbol{\omega}^{*} \times[I] \boldsymbol{\omega}^{*}\right)}{\left\|[I] \boldsymbol{\omega}^{*}\right\| M_{\max }}
$$

To verify that the derivative in Eq. 4.20 along with the $\boldsymbol{\lambda}^{*}$ in Eq. 4.11 satisfy the necessary condition for optimality, we plug $\boldsymbol{\lambda}^{*}$ from Eq. 4.11 into the necessary condition in Eq. 4.9) to get:

$$
\dot{\boldsymbol{\lambda}}^{*}=\frac{[I] \boldsymbol{\omega}^{*} \times[I] \boldsymbol{\omega}^{*}-[I]^{-1}\left(\boldsymbol{\omega}^{*} \times[I] \boldsymbol{\omega}^{*}\right)}{\left\|[I] \boldsymbol{\omega}^{*}\right\| M_{\max }}=-\frac{[I]^{-1}\left(\boldsymbol{\omega}^{*} \times[I] \boldsymbol{\omega}^{*}\right)}{\left\|[I] \boldsymbol{\omega}^{*}\right\| M_{\max }}
$$

Comparing Eq. (4.20) and Eq. 4.21), it can be concluded that the solution $\boldsymbol{\lambda}^{*}$ in Eq. 4.11 satisfies the necessary conditions for optimality. This completes the proposition proof.

Remark 4.1: The optimal control solution is valid only $\forall t \in\left[0, t_{f}\right)$. The optimal control in Eq. (4.8) is defined only when $\beta^{*} \neq 0$. However, if there is at least one interval $[t 1, t 2]$ such that $\beta^{*}=0 \forall t \in[t 1, t 2]$, then this interval $[t 1, t 2]$ is a singularity interval. For a time-optimal control system to be singular, the necessary and sufficient conditions imply that the system must be uncontrollable [87]. On the other hand, for inclined orbits, the spacecraft sees a variation in the magnetic field that is sufficient to guarantee the stability and controllability of the spacecraft [2, 57]. Therefore, for inclined orbits, the system is controllable and is a Normal Time-Optimal Control system (NTOCs) [87]. If $\beta^{*}=0$, this will be only a switching point. 
Remark 4.2: The ambient field vector is time dependent; hence the Hamiltonian is also time dependent. The Hamiltonian is then not a constant along the optimal trajectory; this can be demonstrated by substituting Eqs. (4.11) and (4.12) into Eq. (4.3).

Remark 4.3: The obtained solution is the most effective. This can be seen from Eq. (4.12) where the resulting $\mathbf{M}^{*}$ is orthogonal to the ambient magnetic field $\mathbf{B}$. Hence the resulting torque $\mathbf{T}=\mathbf{M}^{*} \times \mathbf{B}$ is the maximum torque that can be obtained from this $\mathbf{M}^{*}$ - the angle between $\mathbf{M}^{*}$ and $\mathbf{B}$ is $90^{\circ}$, as shown in fig.1.1. Therefore, the Triple Orthogonality Condition among the ambient magnetic field, generated dipole moment and spacecraft applied torque vectors can be considered here as a condition for minimum-time optimal solution.

\section{Stability analysis}

From a stability point of view, the equilibrium point is considered at $\boldsymbol{\omega}^{*}=0$. Therefore, the spacecraft angular kinetic energy can be used as a Lyapunov candidate function for stability proof as follows:

$$
V\left(\boldsymbol{\omega}^{*}\right)=\frac{1}{2} \boldsymbol{\omega}^{* T} \boldsymbol{\omega}^{*}
$$


The Lyapunov candidate function $V(0)=0$ and $V\left(\boldsymbol{\omega}^{*}\right)$ is positive definite elsewhere. The time derivative of the Lyapunov function is:

$$
\dot{V}\left(\boldsymbol{\omega}^{*}\right)=\boldsymbol{\omega}^{* T} \dot{\omega}^{*}
$$

Using Eq. 4.13 along with the effect of the disturbance torque $\mathbf{T}_{D}$ and magnetic rods duty cycle actuation through the function $f$ in Eq. 3.10 , the time derivative of the Lyapunov function is:

$$
\dot{V}\left(\boldsymbol{\omega}^{*}\right)=-f \frac{\boldsymbol{\omega}^{* T}\left[\Gamma_{a v g}\right] \boldsymbol{\omega}^{*}}{\left\|\mathbf{B} \times[I] \boldsymbol{\omega}^{*}\right\|} M_{\max }+\boldsymbol{\omega}^{* T} \mathbf{T}_{D}
$$

where $\left[\Gamma_{a v g}\right]=[\mathbf{B}]_{x}[\mathbf{B}]_{x}^{T}$ is a positive definite matrix as discussed earlier, see Eq. (3.14) [10, 42, 57].

Remark 4.4: In case of ignoring the disturbance torque $\mathbf{T}_{D}=0$, the derivative of the Lyapunov function $\dot{V}$ is negative semi definite. The derivative of the Lyapunov function $\dot{V}\left(\boldsymbol{\omega}^{*}\right)$ equals zero in three situations. The favorable one is when the spacecraft angular velocity $\boldsymbol{\omega}=0$, while the other two situations are when the spacecraft angular momentum vector is parallel to the ambient magnetic field vector or $f=0$ during the desaturation and magnetometer measurement periods. The second situation is not possible to continue for long time because the system is time-varying, as discussed in Remark 4.2. Therefore, The derivative of the Lyapunov function $\dot{V}\left(\boldsymbol{\omega}^{*}\right)$ 
is equal to zero only if the spacecraft angular velocity $\boldsymbol{\omega}^{*}$ is equal to zero or $f=0$ in which there is no control authority over the spacecraft. Therefore, the system will be stable in the sense of the Lyapunov stability theory, yet not asymptotically stable.

Remark 4.5: For bounding disturbance torque assumption, with replacement of $f_{\text {avg }}$ instead of $f$, once the spacecraft angular velocity becomes low, which typically occurs at the end of the detumbling maneuver, the second term on the right hand side of Eq. (4.24) could lead to growing the angular velocity. However, the first term on the right hand side of Eq. (4.24) is negative semi definite. Which implies that, once growing the angular velocity due to the disturbance torque, the first term will dominate again and the angular velocity will be bounded as the kinetic energy will decay again.

As shown in Eq. 4.22), the Lyapunov candidate function $V(0)=0$ and $V\left(\boldsymbol{\omega}^{*}\right)$ is positive definite elsewhere. Equation (4.24) shows that the derivative of the Lyapunov function $\dot{V}\left(\boldsymbol{\omega}^{*}\right) \leq 0$; hence $\dot{V}$ is negative semi-definite and the system is Lyapunov stable, see Remark 4.4. Since the Lyapunov function $V>0 \forall \boldsymbol{\omega}^{*} \neq 0, V=0$ for $\left(\boldsymbol{\omega}^{*}=0\right)$, and $\dot{V}(0)=0$ along with replacing $f$ with its average value $f_{\text {avg }}$ Eq. (3.15), then by LaSalle's global invariant set theorem, the system origin can be claimed to be asymptomatically stable. This completes the stability proof. 


\subsubsection{Case 2: Using Only Ambient Magnetic Field Measure- ments}

In this section, the magnetic field measurements are the only available measurements. By following the discussion in section 3.1.1, where the detumbling law that uses the derivative of the ambient magnetic field can be considered as a tracking problem. In which the spacecraft at the end of the detumbling maneuver will have rotation rate around $2 \Omega$ about the axis of maximum inertia.

In this analysis, the optimization problem is formulated such that the target spacecraft angular velocity at terminal time is set to spin the spacecraft about its maximum inertia axis, with a rate equal to double the orbital rate $\Omega$. Without loss of generality, the spacecraft is assumed axisymmetric, which is the case in most small spacecraft, in which the third axis is the axis of maximum inertia $I_{z}$. Then $\tilde{\boldsymbol{\omega}}$ can be formulated such that:

$$
\tilde{\boldsymbol{\omega}}=\boldsymbol{\omega}-\boldsymbol{\omega}_{s s}=\boldsymbol{\omega}-\left[\begin{array}{lll}
0 & 0 & 2 \Omega
\end{array}\right]^{T},
$$

where $\boldsymbol{\omega}_{s s}$ is the target steady state angular velocity. Hence the target of optimal 
control is to bring $\tilde{\boldsymbol{\omega}}$ to zero. The rigid body equation of motion in terms of $\tilde{\boldsymbol{\omega}}$ is:

$$
\begin{aligned}
\dot{\tilde{\boldsymbol{\omega}}} & =-[I]^{-1}\left(\tilde{\boldsymbol{\omega}} \times[I] \tilde{\boldsymbol{\omega}}+\tilde{\boldsymbol{\omega}} \times[I] \boldsymbol{\omega}_{s s}\right) \\
& +[I]^{-1}\left(-\boldsymbol{\omega}_{s s} \times[I] \tilde{\boldsymbol{\omega}}-\boldsymbol{\omega}_{s s} \times[I] \boldsymbol{\omega}_{s s}+[\mathbf{B}]_{x}^{T} \mathbf{M}\right) \\
& =-[I]^{-1}(\tilde{\boldsymbol{\omega}} \times[I] \tilde{\boldsymbol{\omega}})-[Z] \tilde{\boldsymbol{\omega}}+[I]^{-1}[\mathbf{B}]_{x}^{T} \mathbf{M}
\end{aligned}
$$

where

$$
\begin{gathered}
\dot{\boldsymbol{\omega}}_{s s}=0 \\
\boldsymbol{\omega}_{s s} \times[I] \boldsymbol{\omega}_{s s}=0 \\
{[Z]=\left[\begin{array}{ccc}
0 & 2 \frac{I_{z}-I_{y}}{I_{x}} \Omega & 0 \\
2 \frac{I_{x}-I_{z}}{I_{y}} \Omega & 0 & 0 \\
0 & 0 & 0
\end{array}\right]}
\end{gathered}
$$

The Pontryagin Minimum Principle is conducted to solve the optimal control problem. Using Eqs. (4.26) and (4.1), the Hamiltonian $H \in \mathbb{R}^{1}$ of the system can be written as [87, 88]: 


$$
\begin{aligned}
H(\tilde{\boldsymbol{\omega}}(t), \boldsymbol{\lambda}(t), \mathbf{M}(t)) & =1-\boldsymbol{\lambda}(t)^{T}[I]^{-1}(\tilde{\boldsymbol{\omega}}(t) \times[I] \tilde{\boldsymbol{\omega}}(t)) \\
& -\boldsymbol{\lambda}(t)^{T}[Z] \tilde{\boldsymbol{\omega}}(t)+\boldsymbol{\lambda}(t)^{T}[I]^{-1}[\mathbf{B}(t)]_{x}^{T} \mathbf{M}(t)
\end{aligned}
$$

The arguments are dropped for simplification. The optimal control solution can be obtained by following the same procedure as in the previous section. The resulting optimal control is the same as in Eq. 4.8, where $\boldsymbol{\omega}^{*}$ in Eq. 4.4 is replaced by $\tilde{\boldsymbol{\omega}}^{*}$.

The necessary conditions for optimality are:

1. The Lagrange multiplier $\boldsymbol{\lambda}$ must satisfy:

$$
\dot{\boldsymbol{\lambda}}=-\frac{\partial H}{\partial \tilde{\boldsymbol{\omega}}}=\left([I \tilde{\boldsymbol{\omega}}]_{x}-[I][\tilde{\boldsymbol{\omega}}]_{x}\right)[I]^{-1} \boldsymbol{\lambda}+[Z] \boldsymbol{\lambda}
$$

2. The states must satisfy the following condition:

$$
\dot{\tilde{\boldsymbol{\omega}}}=\frac{\partial H}{\partial \boldsymbol{\lambda}}=-[I]^{-1}(\tilde{\boldsymbol{\omega}} \times[I] \tilde{\boldsymbol{\omega}})-[Z] \tilde{\boldsymbol{\omega}}-[I]^{-1}[\mathbf{B}]_{x}^{T} \frac{[\mathbf{B}]_{x}[I]^{-1} \boldsymbol{\lambda}}{\left\|\mathbf{B} \times[I]^{-1} \boldsymbol{\lambda}\right\|} M_{\max }
$$


Proposition 4.2: The co-state definition given in Eq. 4.33 satisfies the necessary conditions of optimality given in Eq. 4.31) and Eq. (4.32).

$$
\boldsymbol{\lambda}^{*}=\frac{[I]^{2} \tilde{\boldsymbol{\omega}}^{*}}{\left\|[I] \tilde{\boldsymbol{\omega}}^{*}\right\| M_{\max }}
$$

Proof: Substituting the proposed solution in Eq. 4.33 into the control law in Eq. (4.8), we obtain the following expression for the optimal control:

$$
\mathbf{M}^{*}=-\frac{\mathbf{B} \times[I] \tilde{\boldsymbol{\omega}}^{*}}{\left\|\mathbf{B} \times[I] \tilde{\boldsymbol{\omega}}^{*}\right\|} M_{\max }
$$

and the optimal trajectory of the state variable $\tilde{\boldsymbol{\omega}}^{*}$ can be obtained by plugging Eq.4.33) into Eq.44.32):

$$
\dot{\tilde{\boldsymbol{\omega}}}^{*}=-[I]^{-1}\left(\tilde{\boldsymbol{\omega}}^{*} \times[I] \tilde{\boldsymbol{\omega}}^{*}\right)-[Z] \tilde{\boldsymbol{\omega}}^{*}-[I]^{-1}[\mathbf{B}]_{x}^{T} \frac{\mathbf{B} \times[I] \tilde{\boldsymbol{\omega}}^{*}}{\left\|\mathbf{B} \times[I] \tilde{\boldsymbol{\omega}}^{*}\right\|} M_{\max }
$$

In order to check if the proposed solution in Eq. 4.33), along with Eq. 4.34 and Eq. 4.35, satisfy the necessary conditions of optimality, we start by taking the derivative of Eq. (4.33):

$$
\dot{\lambda}^{*}=\overbrace{\frac{[I]^{2} \dot{\tilde{\boldsymbol{\omega}}}^{*}\left\|[I] \tilde{\boldsymbol{\omega}}^{*}\right\| M_{\max }}{\left(\left\|[I] \tilde{\boldsymbol{\omega}}^{*}\right\| M_{\max }\right)^{2}}}^{\mathbf{T}_{1}}-\overbrace{\frac{[I]^{2} \tilde{\boldsymbol{\omega}}^{*} \tilde{\boldsymbol{\omega}}^{* T}[I]^{2} \dot{\tilde{\boldsymbol{\omega}}}^{*} M_{\text {max }}}{\left\|[I] \tilde{\boldsymbol{\omega}}^{*}\right\|^{3} M_{\max }^{2}}}^{\mathbf{T}_{2}}
$$


Substituting for $\dot{\tilde{\omega}}^{*}$ in the term $\mathbf{T}_{2}$ from Eq. 4.35, we get:

$$
\begin{aligned}
\mathbf{T}_{2} & =-\frac{[I]^{2} \tilde{\boldsymbol{\omega}}^{*} \tilde{\boldsymbol{\omega}}^{* T}[I]^{2}\left([I]^{-1}\left(\tilde{\boldsymbol{\omega}}^{*} \times[I] \tilde{\boldsymbol{\omega}}^{*}\right)\right)}{\left\|[I] \tilde{\boldsymbol{\omega}}^{*}\right\|^{3} M_{\max }}-\frac{[Z]^{T}[I]^{2} \tilde{\boldsymbol{\omega}}^{*} \tilde{\boldsymbol{\omega}}^{* T}[I]^{2} \tilde{\boldsymbol{\omega}}^{*}}{\left\|[I] \tilde{\boldsymbol{\omega}}^{*}\right\|^{3} M_{\max }} \\
& -\frac{[\Gamma]^{T}[I]^{2} \tilde{\boldsymbol{\omega}}^{*} \tilde{\boldsymbol{\omega}}^{* T}[I]^{2} \tilde{\boldsymbol{\omega}}^{*}}{\left\|[I] \tilde{\boldsymbol{\omega}}^{*}\right\|^{3}\left\|B \times[I] \tilde{\boldsymbol{\omega}}^{*}\right\|}
\end{aligned}
$$

Consider the first term in Eq.4.37):

$$
\left.[I]^{2} \tilde{\boldsymbol{\omega}}^{*} \tilde{\boldsymbol{\omega}}^{* T}[I]^{2}\left([I]^{-1}\left(\tilde{\boldsymbol{\omega}}^{*} \times[I] \tilde{\boldsymbol{\omega}}^{*}\right)\right)=[I]^{2} \tilde{\boldsymbol{\omega}}^{*}\left([I] \tilde{\boldsymbol{\omega}}^{*}\right)^{T}\left(\tilde{\boldsymbol{\omega}}^{*} \times[I] \tilde{\boldsymbol{\omega}}^{*}\right)\right)=0
$$

Also, note that:

$$
\tilde{\boldsymbol{\omega}}^{* T}[I]^{2} \tilde{\boldsymbol{\omega}}^{*}=\left\|[I] \tilde{\boldsymbol{\omega}}^{*}\right\|^{2}
$$

Then the term $\mathbf{T}_{2}$ reduces to:

$$
\mathbf{T}_{2}=-\frac{[Z]^{T}[I]^{2} \tilde{\boldsymbol{\omega}}^{*}}{\left\|[I] \tilde{\boldsymbol{\omega}}^{*}\right\| M_{\max }}-\frac{[\Gamma]^{T}[I]^{2} \tilde{\boldsymbol{\omega}}^{*}}{\left\|[I] \tilde{\boldsymbol{\omega}}^{*}\right\|\left\|\mathbf{B} \times[I] \tilde{\boldsymbol{\omega}}^{*}\right\|}
$$


Substituting for $\dot{\tilde{\omega}}^{*}$ in the term $\mathbf{T}_{1}$ from Eq. (4.35), we get:

$$
\mathbf{T}_{1}=-\frac{[I]^{-1}\left(\tilde{\boldsymbol{\omega}}^{*} \times[I] \tilde{\boldsymbol{\omega}}^{*}\right)}{\left\|[I] \tilde{\boldsymbol{\omega}}^{*}\right\| M_{\max }}-\frac{[Z]^{T}[I]^{2} \tilde{\boldsymbol{\omega}}^{*}}{\left\|[I] \tilde{\boldsymbol{\omega}}^{*}\right\| M_{\max }}-\frac{[\Gamma]^{T}[I]^{2} \tilde{\boldsymbol{\omega}}^{*}}{\left\|[I] \tilde{\boldsymbol{\omega}}^{*}\right\|\left\|\mathbf{B} \times[I] \tilde{\boldsymbol{\omega}}^{*}\right\|}
$$

Substituting the terms $\mathbf{T}_{1}$ and $\mathbf{T}_{2}$ from Eq. 4.41) and 4.40, respectively, into Eq. (4.36), The derivative of the co-state variable $\boldsymbol{\lambda}^{*}$ reduces to:

$$
\dot{\boldsymbol{\lambda}}^{*}=-\frac{[I]^{-1}\left(\tilde{\boldsymbol{\omega}}^{*} \times[I] \tilde{\boldsymbol{\omega}}^{*}\right)}{\left\|[I] \tilde{\boldsymbol{\omega}}^{*}\right\| M_{\max }}
$$

Using the definition of $\boldsymbol{\lambda}^{*}$ in Eq. (4.33), it is straightforward to show that $\boldsymbol{\lambda}^{* T}[Z] \tilde{\boldsymbol{\omega}}^{*}=$ 0 ; consequently its partial derivative $\frac{\partial\left(\boldsymbol{\lambda}^{* T}[Z] \tilde{\boldsymbol{\omega}}^{*}\right)}{\partial \tilde{\boldsymbol{\omega}}^{*}}=0$. Moreover, it is straightforward to show that $\frac{\partial \boldsymbol{\lambda}^{*}}{\partial \tilde{\boldsymbol{\omega}}^{*}}=0$ and $\frac{\partial M^{*}}{\partial \tilde{\boldsymbol{\omega}}^{*}}=0$. Therefore the term $[Z] \lambda^{*}$ vanishes in Eq. 4.31). Finally, it is possible to show that the proposed co-state solution, Eq. (4.33) satisfies the necessary condition for optimality by substituting Eq. 4.33 into the necessary condition for optimality, Eq. (4.31). And comparing the obtained results with Eq. (4.42). This completes the proposition proof.

Using (3.7), the time-optimal B-dot law in Eqs. 4.34 becomes:

$$
\mathbf{M}^{*}=-\frac{\mathbf{B} \times[I][\Sigma]^{-1} \dot{\mathbf{B}}}{\left\|\mathbf{B} \times[I][\Sigma]^{-1} \dot{\mathbf{B}}\right\|} M_{\max }
$$




\section{Stability Analysis}

The stability of the proposed B-dot law (refereed to as PMP B-dot) can be analyzed using a Lyapunov approach. The Lyapunov candidate function is selected as:

$$
V 2\left(\tilde{\boldsymbol{\omega}}^{*}\right)=\frac{1}{2} \tilde{\boldsymbol{\omega}}^{* T} \tilde{\boldsymbol{\omega}}^{*}
$$

Note that $V 2(0)=0$, and that $V 2\left(\tilde{\boldsymbol{\omega}}^{*}\right)$ is positive definite elsewhere. The time derivative of the Lyapunov function is:

$$
\dot{V} 2\left(\tilde{\boldsymbol{\omega}}^{*}\right)=\tilde{\boldsymbol{\omega}}^{* T} \dot{\tilde{\boldsymbol{\omega}}}^{*}
$$

Using Eqs. (4.35) and (3.14) along with intermittent actuation of the magnetic rods through the function $f$ Eq. (3.9), the time derivative of the Lyapunov function becomes:

$$
\dot{V} 2\left(\tilde{\boldsymbol{\omega}}^{*}\right)=-f \frac{\tilde{\boldsymbol{\omega}}^{* T}\left[\Gamma_{a v g}\right] \tilde{\boldsymbol{\omega}}^{*}}{\left\|\mathbf{B} \times[I] \tilde{\boldsymbol{\omega}}^{*}\right\|} M_{\max }+\tilde{\boldsymbol{\omega}}^{* T} \mathbf{T}_{D}
$$

where the matrix $\left[\Gamma_{\text {avg }}\right]$ is positive definite [10, 42, 57]. Note that in deriving Eq. (4.46), we utilized the fact that $\tilde{\boldsymbol{\omega}}^{* T}[I][Z] \tilde{\boldsymbol{\omega}}^{*}=0$, since the spacecraft is axisymmetric $\left(I_{x}=I_{y}\right)$. It is noted that Remarks 4.1, 4.2, 4.4 and 4.5 are applicable also for this case. 
As shown in Eq. 4.44), the Lyapunov candidate function $V(0)=0$ and $V\left(\tilde{\boldsymbol{\omega}}^{*}\right)$ is positive definite elsewhere. Eq (4.46) shows that the derivative of the Lyapunov function $\dot{V}\left(\tilde{\boldsymbol{\omega}}^{*}\right) \leq 0$; hence $\dot{V}$ is negative semi-definite and the system is Lyapunov stable. Since the Lyapunov function $V>0 \forall \tilde{\boldsymbol{\omega}}^{*} \neq 0, V=0$ for $\left(\tilde{\boldsymbol{\omega}}^{*}=0\right)$, and $\dot{V}(0)=$ 0 along with replacing $f$ with its average value $f_{\text {avg }}$ Eq. (3.15), then by LaSalle's global invariant set theorem, the system origin can be claimed asymptomatically stable. This completes the stability proof.

Remark 4.6: The obtained solution in Eq. 4.43 guarantees the TOC among the ambient magnetic field, the magnetic toque, and dipole moment vectors in the ideal case. Which is not the case for conventional B-dot law [26], and also in its variants [27 31] as discussed before.

\subsection{Simulation Results with Discussion}

In order to assess the performance of the two time-optimal detumbling controllers, the results of simulation of these two controllers are compared to the $\boldsymbol{\omega}$ law in Eq. 3.4 and B-dot law in Eq. (3.1) 1]. The developed optimal-Based angular velocity control law Eq. 4.12 will be refereed to as (PMP $\boldsymbol{\omega}$ law), whereas The developed optimalBased B-dot control law Eq. (4.43) will be refereed to as (PMP B-dot law). Same parameters and simulation environment that used in section 3.3 are used here except 
$I_{z}=3.264\left[g \cdot m^{2}\right]$ and $\chi=1 e^{-9}$ is selected. The consumed electric energy vector $\mathbf{E}$ will be used instead $\mathbf{t}_{o n}$ and is computed as follows:

$$
\mathbf{E}=\delta T_{s} \mathbf{V} \odot \mathbf{i}_{c},
$$

where $\mathbf{V}$ is the voltage vector for the magnetic rods and for simplification it will be equal to one volt during the simulation.

\section{A Case Study}

Results from a sample case will be rendered first before presenting the statistical Monte Carlo analysis. Figure 4.1 represents the spacecraft angular velocity magnitude history during the detumbling maneuver. The PMP B-dot law in Eq. 4.43 along with the five-stencil method as a numerical derivative tool for computing the magnetic field derivative, is labeled (PMP B-dot law (5pt)). As shown in fig 4.1, the proposed optimal control laws are faster in detumbling. Figure 4.1 also shows that the Bdot laws are slower in detumbling than the controllers that use the angular velocity directly [66]. The reasons for that are as follows; the errors in the magnetometers [62], the numerical errors in computing the magnetic field derivative [62], and ignoring the effect of the first term in Eq. (3.3) in the B-dot law in [1].

Figure 4.2 shows the magnitude of the control history for the four controllers. The 


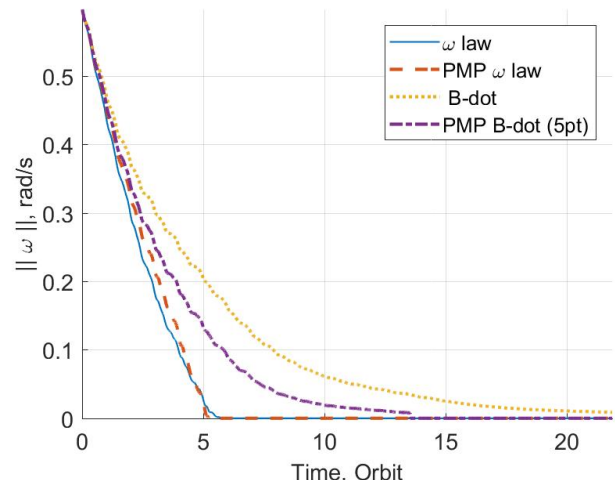

Figure 4.1: Angular velocities magnitude history

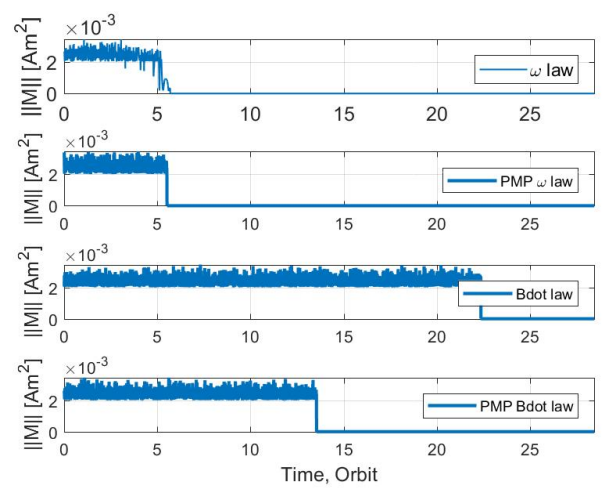

Figure 4.2: History of the dipole moment magnitude

control effort of the B-dot law, see the third subplot fig. 4.2, is at its maximum permissible effort. The reason for that, as mentioned early in sections 1.2 .2 and 3.2 . due to the magnetometer errors, is the computed $\dot{\mathbf{B}}$ will give a controller command needs to be saturated. This in turn lead to electric energy waste. The PMP B-dot control is still able to preserve the angular velocity at small values as shown in the upper three subplots in fig. 4.3. The lower subplot of fig. 4.3 is a zoom of the angular velocity at the third axis, the axis of maximum inertia. It is clear that the spacecraft angular velocity is within double the orbital rate which is compatible with the desired angular velocity $\tilde{\boldsymbol{\omega}}$ in section 4.1 .2 .

\section{Monte Carlo Analysis}

An extensive 4,000 Monte Carlo runs' results are presented; 1,000 runs for each controller. For each run, different noises seeds are used assuming Gaussian distribution. 

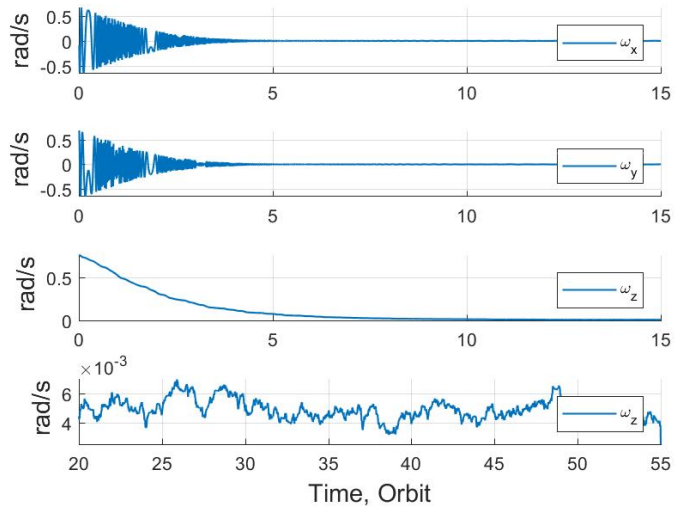

Figure 4.3: Angular velocity history for the PMP B-dot controller

In order to investigate the impact of the different initial conditions, each run has its initial angular rates. These values are generated randomly and are the same for all controllers. The results are reported for the consumed electric energy by the magnetic rods, and for the detumbling time.

\section{Monte Carlo results for angular velocity controllers}

Figure 4.4 shows the histogram and distribution of improvement in $t_{\text {det }}$ by using PMP $\boldsymbol{\omega}$ law Compared to $\boldsymbol{\omega}$ law. Figure 4.4 shows that the mean value of detumbling time reduction for the 1000 different runs is about 4.02\%, whereas the standard deviation is $2.86 \%$.

Figure 4.5 shows the histogram and normal distribution of $\mathbf{E}$ of these Monte Carlo runs. The mean increase in $\mathbf{E}$ consumed by the PMP $\boldsymbol{\omega}$ law is $7.3 \%$ with standard deviation $3.86 \%$. The results of this section prove that the $\boldsymbol{\omega}$ law in [1] is sub-optimal 


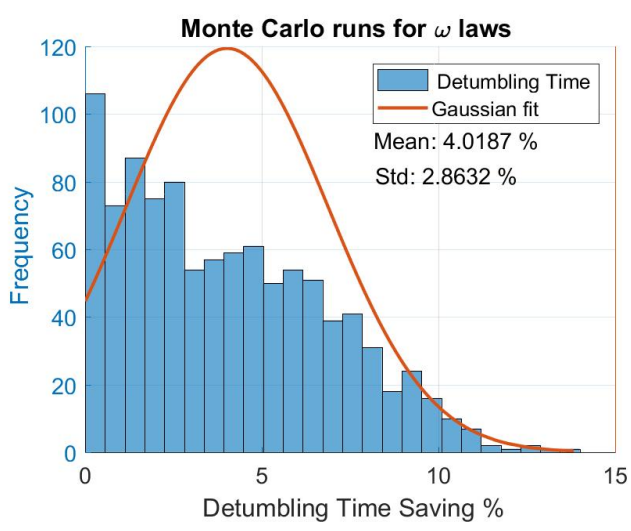

Figure 4.4: Histogram represents the $t_{\text {det }}$ reduction percentage for the PMP $\boldsymbol{\omega}$ law compared to the $\boldsymbol{\omega}$ law [1]

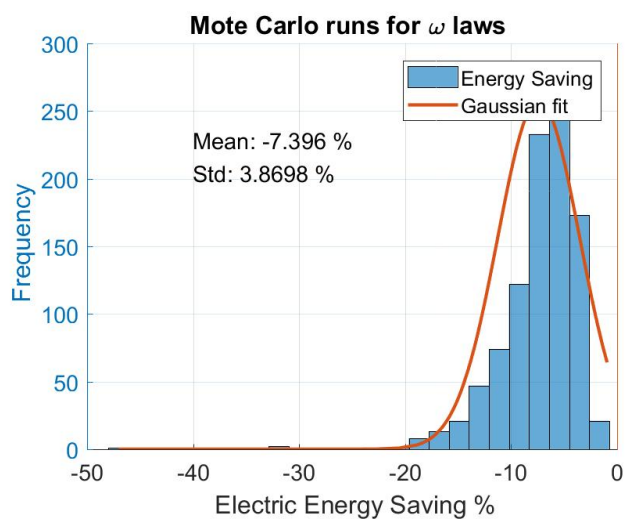

Figure 4.5: Histogram represents the $\mathbf{E}$ reduction percentage for the PMP $\boldsymbol{\omega}$ law compared to the $\boldsymbol{\omega}$ law [1]

as the authors of [1] also report.

\section{Monte Carlo results for B-dot controllers}

In this part, the results of the PMP B-dot law compared to the results from the B-dot law in reference [1] are presented and discussed. For the 1000 runs, the PMP B-dot law is able to detumble the spacecraft in significantly less time as shown in the histogram for the improvement in $t_{d e t}$ fig. 4.6. The mean reduction in the $t_{d e t}$ is $42.58 \%$ and the standard deviation $4.46 \%$.

Due to ignoring the effect of the first term on the right hand side of Eq. (3.3), the TOC is not guaranteed for the B-dot law in [1]. Adding to that the more accurate calculations of the magnetic field derivative in the proposed algorithm. The results show a significant savings in power consumption by the magnetic rods when using 


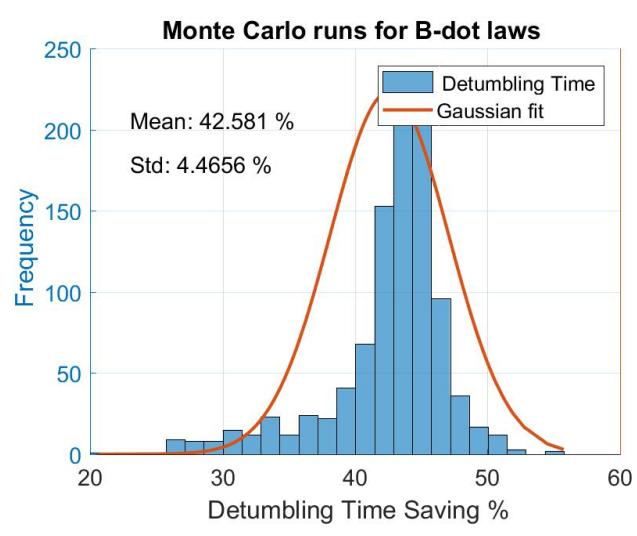

Figure 4.6: Histogram represents the $t_{\text {det }}$ reduction percentage for the PMP B-dot law compared to the B-dot law [1]

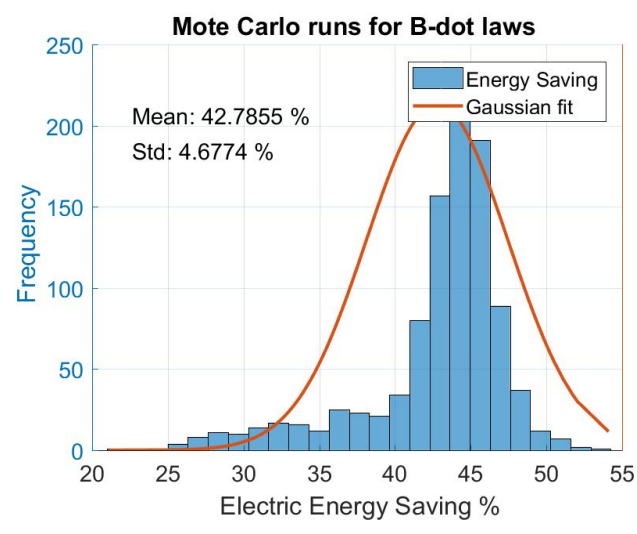

Figure 4.7: Histogram represents the E reduction percentage for the PMP Bdot law compared to the B-dot law [1]

the PMP B-dot law as shown in fig 4.7. The mean electric energy saving is $42.78 \%$ with a standard deviation of $4.67 \%$.

\section{A Special Case study}

The variation of the magnetic field is weak at inclination angles near the equator and is strong at inclination angles near the polar. Moreover, for high altitude, the magnetic field strength is weak and is strong at low altitude orbits.

Here, the spacecraft initial angular velocity is fixed while the orbit altitude is varied. The orbit inclination angle is chosen to be $6^{\circ}$. At this inclination, the magnetic field variation is very small, which leads to a significantly increased detumbling time. In general, this low inclination orbit leads to increasing the required time for attitude control and could affect even the controllability of the system, especially for low 


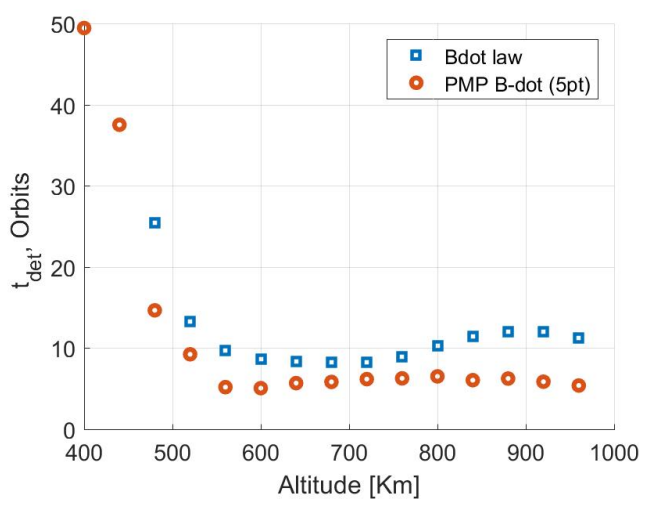

Figure 4.8: Detumbling time for the B-dot law [1] and the PMP B-dot law for different altitudes at orbits of $6^{\circ}$ inclination angle

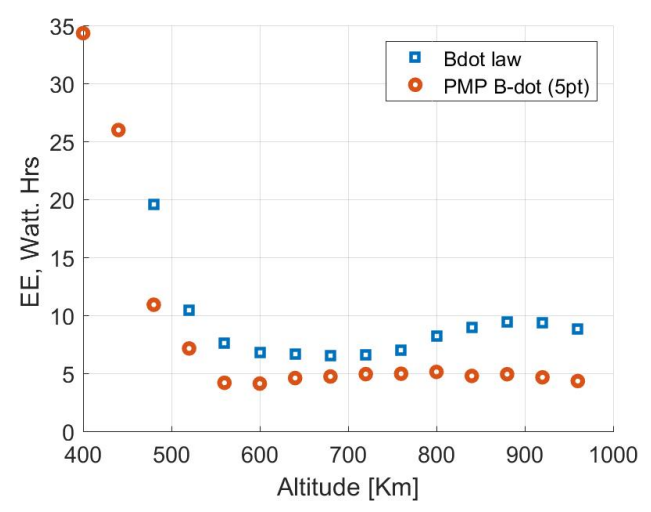

Figure 4.9: Electric energy for the Bdot law [1] and the PMP B-dot law for different altitudes at orbits of $6^{\circ}$ inclination angle

magnetic control effort actuators.

Here it desired to find out altitudes at which an algorithm cannot detumble the spacecraft within a reasonable amount of time (here selected to be 56 orbits.) The maximum expected initial angular velocity in each axis is $\boldsymbol{\omega}_{\max }=9^{\circ}$. Figure 4.8 shows the detumbling time in orbits for both the PMP B-dot law, and the B-dot law in reference [1] versus altitude. The missing points of the B-dot law at altitudes less than $480[\mathrm{~km}]$ mean that the B-dot control is not able to detumble the spacecraft within the required time frame for this scenario. The PMP B-dot does not have this problem. In general, the PMP B-dot law performs better in terms of the detumbling time and power consumption, as can be seen in figs 4.8 and 4.9 , even at low orbit inclination angles.

Another Monte Carlo simulation is conducted to render the magnetic field noise effect 


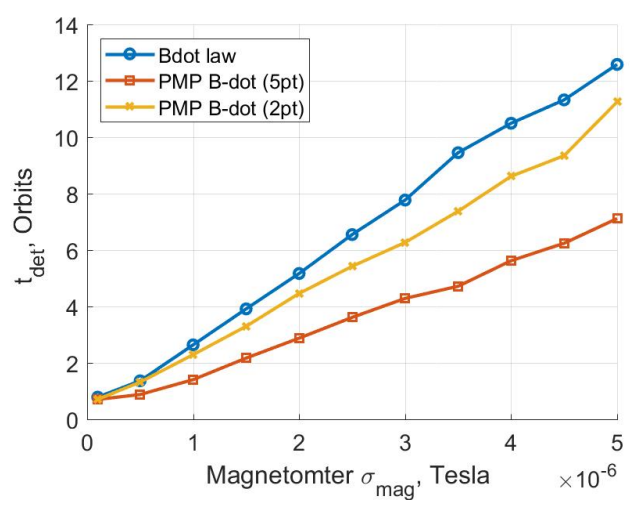

Figure 4.10: Detumbling time for the B-dot law [1] and the PMP B-dot law for different magnetometer noise standard deviation $\sigma_{m a g}$

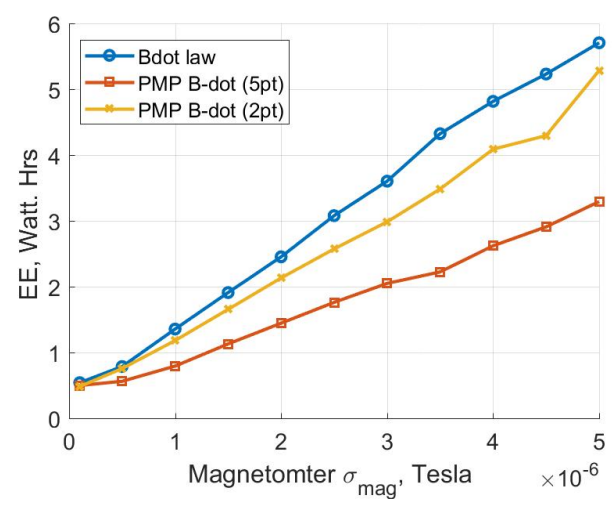

Figure 4.11: Electric energy for the Bdot law [1] and the PMP B-dot law for different magnetometer noise standard deviation $\sigma_{m a g}$

on the PMP B-dot law. The noise is considered here as a white noise with zero mean.

The spacecraft initial angular velocity is fixed while the standard deviation of the noise of the magnetometer is varied to represent the effect of the magnetometer noise.

Foster and Elkaim [54] proposed a nonlinear two-step estimation algorithm for the calibration of solid-state strap down magnetometers. They proved the effectiveness of the proposed algorithm via experimental case results where the standard deviation of the initial un-calibrated magnetometer measurements is $\sigma_{m a g}=1.8408 e^{-6}[$ Tesla $]$. In order to present a realistic situation, the standard deviation of this Monte Carlo runs are selected to vary around this value $\sigma_{m a g}=1.8408 e^{-6}[$ Tesla $]$.

Figure 4.10 shows the mean detumbling time in orbits for both B-dot laws versus magnetometer noise standard deviation $\sigma_{m a g}$ for this Monte Carlo runs. Three lines are shown in fig. 4.10. The first line represents the B-dot law in reference [1], using two 

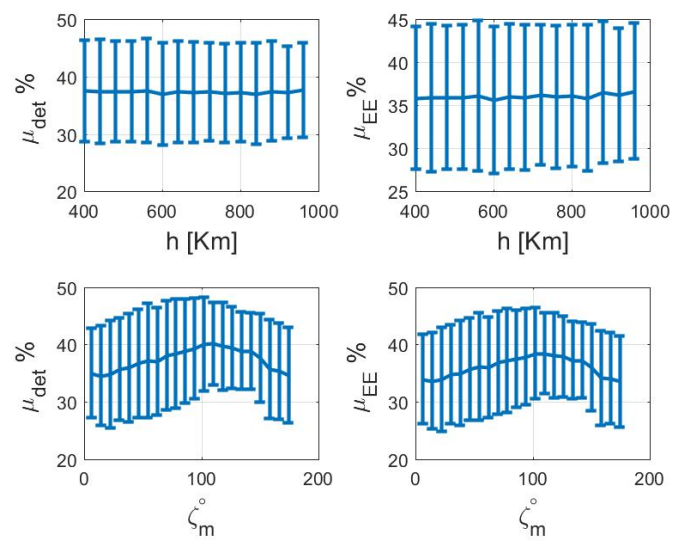

Figure 4.12: Improvement of detumbling time and electric energy consumption versus orbital altitude and inclination

points at each time step for computing the derivative of the magnetic field. The second line represents the PMP B-dot law, using two points at each time step for computing the derivative of the magnetic field. The third line represents the PMP B-dot law, using five points at each time step for computing the derivative of the magnetic field. It is clear that the PMP B-dot gives a better detumbling time performance as the noise standard deviation increases. The performance of both B-dot laws at very low magnetometer noise standard deviation are almost the same. However, this is not a realistic case as pointed out earlier in section 3.2 . Figure 4.11 shows the mean electric energy consumption versus $\sigma_{m a g}$ for this Monte Carlo runs. The results confirm the claimed advantage of the PMP B-dot control.

To emphasize the good performance of the PMP B-dot law, a 110,000 Monte Carlo runs are carried out with different spacecraft moment of inertia $I=$ $\operatorname{diag}\left(\begin{array}{lll}0.33 & 0.33 & 0.37\end{array}\right)\left[\mathrm{kg} \cdot \mathrm{m}^{2}\right]$ and maximum dipole moment magnitude $M_{\max }=$ 
$2\left[A m^{2}\right]$. The orbital parameters are varied. Specifically the orbital inclination $\zeta_{m}$ is between $6^{\circ}$ and $174^{\circ}$, and the orbit altitude $h$ is between $400[\mathrm{~km}]$ to $990[\mathrm{~km}]$ above earth surface. The results are presented on an error plot bar in terms of the mean improvement in the detumbling time $\mu_{\text {det }}$ and the mean improvement in the electric energy consumption $\mu_{E E}$, versus the orbital altitude $h$ and inclination $\zeta_{m}$. The error bars represent the standard deviation and the curves are the mean values. Figure 4.12 shows the four subplots of the error bars to render the performance at different orbital conditions. In all cases, there is a significant improvement when using the PMP B-dot control compared to the B-dot law in [1].

Finally, the 110, 000 Monte Carlo runs are repeated for the TOC B-dot law Eq. (3.8) to compare these results with the ones that obtained for PMP B-dot law Eq. (4.43). The results reported in the two histograms for the $t_{d e t}$ and $\mathbf{E}$ respectively, see figs 4.13 and 4.14. The PMP has always the minimum time. Yet, also proof that TOC B-dot can be considered as sub-optimal minimum time controller. 


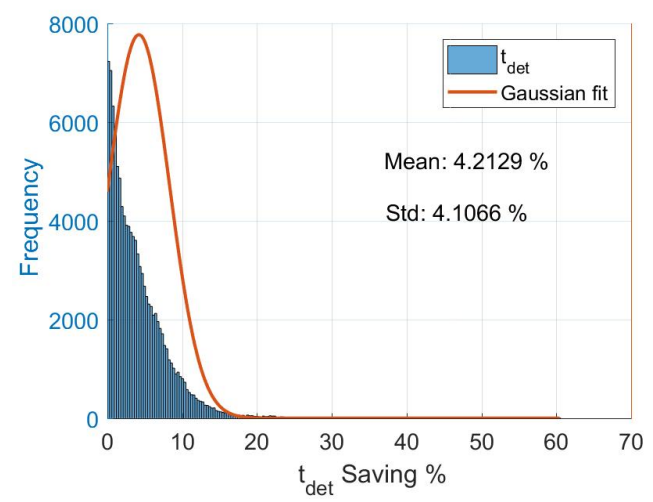

Figure 4.13: Histogram represents the $t_{\text {det }}$ reduction percentage for the PMP B-dot law Eq.4.43 compared to the TOC B-dot law Eq. (3.8)

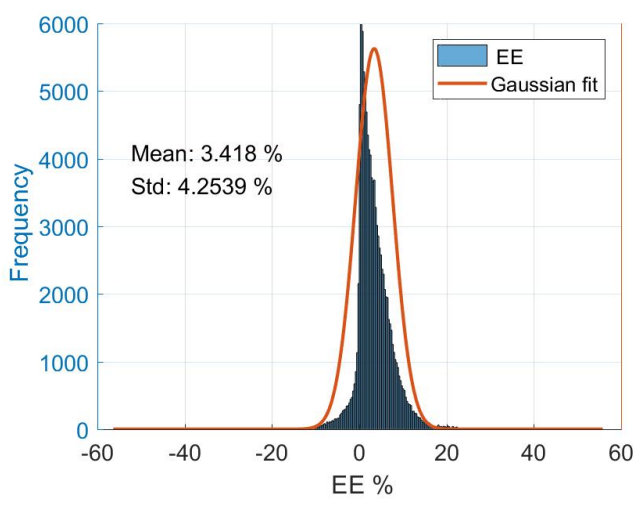

Figure 4.14: Histogram represents the E reduction percentage for the PMP Bdot law Eq.4.43 compared to the TOC B-dot law Eq.(3.8)

\subsection{Conclusion}

Two time-optimal control laws are derived from the optimality necessary conditions using the Pontryagin Minimum Principal where the control effort is constrained. The first control law assumes the availability of the spacecraft angular velocity and the ambient magnetic field measurements in the feedback loop, while the second control law assumes the availability of only the ambient magnetic field measurements. The second optimal control law is considered a new variant of the classical B-dot law. Both controllers maintain the magnetic dipole moment vector in the plane perpendicular to the ambient magnetic field; a condition that most of the B-dot control algorithms do not guarantee.

The proposed control laws are compared to recently developed detumbling control 
laws from the literature. Monte Carlo simulations are conducted to carry out these comparisons. The results of the numerical simulations demonstrate that the proposed time-optimal control laws achieve faster detumbling maneuvers. In terms of power consumption, the proposed B-dot control consumes significantly less power compared to the reference B-dot law. Further comparison with the proposed B-dot law in the previous chapter (TOC B-dot law) that verifies the time optimality of the developed optimal-based B-dot law (PMP B-dot law). 


\section{Chapter 5}

\section{Improved Spacecraft Magnetic}

\section{Attitude Maneuvering}

A magnetometer is essential in spacecraft magnetic attitude control due to the need for magnetic field information in the feedback to compute the control command. The measurements of the magnetometer, however, are usually affected by other electric currents in the spacecraft, especially those of the magnetic coils when they are turned on during actuation. As a result, magnetic rods and magnetometers are usually turned on at alternate times, resulting in a reduced duty cycle of the magnetic rods, and hence longer maneuver times. This chapter presents a magnetic attitude control system with extended duty cycle and low magnetometer measurements frequency. Instead of the real measurements, a computed magnetic field pseudo measurement vector is used for 
updating the control command during the duty cycle. Using the measured spacecraft rotational motion, and knowing the control torque command, it is possible to compute the magnetic field pseudo measurement vector. These computations are corrected using magnetometer measurements at a lower rate. The Tikhonov regularization approach is implemented to solve the singular magnetic torque system. Real data obtained from the CASSIOPE spacecraft are used for validation of the proposed approach.

\subsection{Magnetic Rods Duty Cycle Extension}

As discussed in chapter 1, the magnetic rods and the magnetometers are turned on at alternate times so as to avoid high noise on the magnetometers measurements if turned on while the magnetic rods are active. This results in the magnetic rods being operated with a certain duty cycle; this issue is not always addressed in the literature where most studies assume continuous operation of both the magnetic rods and the magnetometers $[24,61,63,89,90]$. This section highlights the significance of the proposed control scheme in which the target is to increase the magnetic rods duty cycle. In the following discussion and in the simulation results, the current is assumed constant; this is the case in most practical implementations due to the easiness of implementation and the less complexity of the magnetic rods design. 


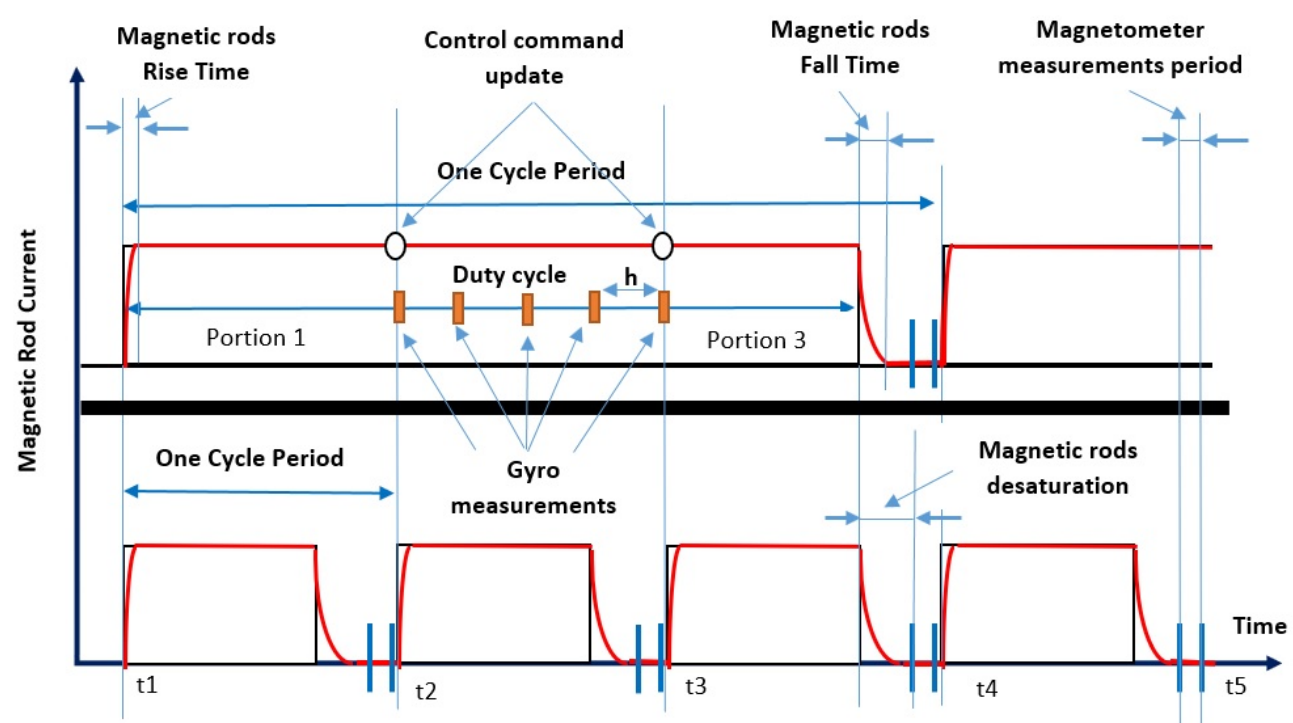

Figure 5.1: The magnetic rods operate on a duty cycle, alternating with the magnetometers to avoid high noise on magnetometers.

The lower part fig. 5.1 represents the nominal operation of the intermittent actuation of the magnetic rods and desaturation and magnetometer measurements periods. This intermittent activation is discussed before in fig. 1.3 and section 1.2 .2 . This operation will be considered here as the "reference" operation sequence between activating the magnetic rods and magnetic field measurements for the constant current pulse width implementation case.

Control algorithms that do not account for the above duty cycle usually underestimate the maneuver time; moreover the simulated power consumption by the magnetic rods is observed in many cases to exceed the computed one when the duty cycle is neglected. Intuitively, increasing the duty cycle ratio $\delta$ would reduce the gap between the two cases. This is the motivation of this study in this chapter and the 
following one. An algorithm is developed to increase the duty cycle by computing the ambient magnetic field parameters to be used for control command update and hence eliminates the need for magnetic field measurements at some cycles. For example, the upper part of fig. 5.1 illustrates a scenario where the magnetic field is measured every three cycles, while counting on the "computed magnetic field or what we will call later magnetic field pseudo measurement" in computing the control command, in the cycles that do not have real magnetometers measurements. As a result, a longer duty cycle for the magnetic rods becomes possible. The equivalent duty cycle ratio $\bar{\delta}$ for the proposed algorithm can be computed as follows:

$$
\bar{\delta}=1-\frac{1-\delta}{\epsilon}
$$

where $\epsilon$ is a constant real positive number, $\epsilon>1$, and it is a design parameter. The upper part of fig. 5.1 represents the case where $\epsilon=3$. The magnetometers are turned on only at the end of each third cycle; clearly the magnetic rods have longer duty cycle. For example, if $\delta=0.7$, then the duty cycle of the proposed algorithm would be $\bar{\delta}=0.9$, see Eq. (5.1). Without loss of generality, the frequency $f_{c}$ of updating the control command is kept fixed at the rate $f_{c}=1 / T_{s}$ in this study for comparison purpose. 


\subsection{Magnetic Field Pseudo Measurement}

A core algorithm that computes the ambient magnetic field pseudo measurement when magnetometer measurements are not available is crucial for the implementation of the proposed approach as described in section 5.1. This section describes a calculation procedures of this magnetic field pseudo measurement. The key concept is that if magnetic dipole $\mathbf{M}$ (control command) is known, and the angular velocity of the spacecraft (rotational response) is measured, then it is possible to determine the ambient magnetic field that interacted with the known command dipole moment and resulted in this known angular velocity. A key equation that is used in these calculations is Eq. 2.7), where $\mathbf{B}$ is unknown, $\mathbf{M}$ is known, and $\mathbf{T}$ can be computed as function of the angular velocity, as briefed in section 5.2.1 below. One obstacle is that the $[\mathbf{M}]_{x}$ in Eq. $(2.7)$ is singular; that is for a given $\mathbf{M}$ and $\mathbf{T}$, the $\mathbf{B}$ is not unique. This chapter presents a regularization approach to overcome this problem; this is detailed in section 5.2 .2 below.

\subsubsection{Torque Computation}

The torque is computed using Euler's equation, Eq. (2.5). In Eq. (2.5), the angular velocity is assumed measured using a gyro, and the time rate of change of the angular 
velocity, $\dot{\boldsymbol{\omega}}$, is computed numerically. The numerical method for computing $\dot{\boldsymbol{\omega}}$ has a significant impact on the accuracy of the obtained results as discussed before in section 1.2.2. Therefore, one-dimensional five-point stencil method is used to evaluate the time rate of change of $\boldsymbol{\omega}$ as follows [86]:

$$
\dot{\boldsymbol{\omega}}_{t-2 h}=\frac{-\boldsymbol{\omega}_{t}+8 \boldsymbol{\omega}_{t-h}-8 \boldsymbol{\omega}_{t-3 h}+\boldsymbol{\omega}_{t-4 h}}{12 h}+\frac{h^{4}}{30} \boldsymbol{\omega}_{t-2 h}^{5}
$$

where $h$ is the time step of the angular velocity measurement, see fig. 5.1. In this study, it is assumed that all the five gyro measurements are collected within $T_{s}$, and hence a constant average torque value is assumed within each $T_{s}$, while computing the derivative over the same cycle period, at the middle point $(t-2 h)$. Therefore, it is assumed in this study that the frequency of collecting the gyro measurements is at least five times higher than the control command frequency update as seen in the upper subplot of fig. 5.1.

\subsubsection{Regularization of The Singular System}

Equation 2.7 is ill posed as there is no unique solution, and the ordinary least squares method cannot be used in this case. The ordinary least squares method seeks 
to minimize the sum of the squares of the residuals, which can be written as:

$$
\text { Minimize }\left\|[\mathbf{M}]_{x} \mathbf{B}-\mathbf{T}\right\|_{2}^{2}
$$

One way to handle this type of ill posed problem is through regularization. The Tikhonov regularization is a commonly used method for regularization of ill-posed problems [91, in which a regularization term, $\alpha^{2}\left\|\mathbf{B}_{\text {sdo }}\right\|_{2}^{2}$, is added to the residual square, and the minimization problem becomes:

$$
\text { Minimize }\left\|[\mathbf{M}]_{x} \mathbf{B}_{\text {sdo }}-\mathbf{T}\right\|_{2}^{2}+\alpha^{2}\left\|\mathbf{B}_{\text {sdo }}\right\|_{2}^{2}
$$

where $\alpha$ is the Tikhonov regularization parameter, which is a real scalar. The $\mathbf{B}$ vector is replaced by the pseudo measurement vector $\mathbf{B}_{s d o}$, and the goal is to achieve $\mathbf{B}_{s d o} \approx$ B. The minimization of the new least squares problem implies the minimization of both the original residual term, and the regularization term. This new least squares problem can be analyzed via singular value decomposition. The matrix $[\mathbf{M}]_{x}$ can be decomposed as follows [92]:

$$
[\mathbf{M}]_{x}=[U][\Lambda][V]^{T}
$$

where $[U] \in \mathbb{R}^{m \times m}$ is unitary matrix, $[\Lambda] \in \mathbb{R}^{m \times n}$ is a diagonal matrix which elements are the singular values of the matrix $[\mathbf{M}]_{x}, \sigma_{i}, i=1,2,3,[V] \in \mathbb{R}^{n \times n}$ is unitary matrix, and $[V]^{T}$ is the conjugate transpose of $[V]$. The solution to this new least squares problem yields an estimate for the magnetic field pseudo measurement vector $\mathbf{B}_{\text {sdo }}$ 
which is expressed as follows [91, 93]:

$$
\mathbf{B}_{s d o}=[V][D][U]^{T} \mathbf{T}
$$

where $\mathbf{T}$ is the computed torque from section 5.2.1, and $[\mathrm{D}]$ is a diagonal matrix which elements are defined as:

$$
D_{i i}=\frac{\sigma_{i}}{\sigma_{i}^{2}+\alpha^{2}}
$$

where the regularization parameter $\alpha$ is to be optimized to minimize both the residual term and the regularization term.

Reference [91] presents another, yet equivalent, form for computing the optimal solution for the type of problem in (5.4), which is here adopted for computing the optimal magnetic field pseudo measurement vector that minimizes (5.4). The optimal estimate for the magnetic field pseudo measurement vector $\mathbf{B}_{\text {sdo }}$ can be written as follows [91]:

$$
\mathbf{B}_{s d o}=\sum_{i=1}^{n} f_{i} \frac{u_{i}^{T} \mathbf{T}}{\sigma_{i}} v_{i}
$$

where $n$ is the number of singular values of $[\mathbf{M}]_{x}$, and $u_{i}, v_{i}$ are the orthogonal left and right singular vectors of $[\mathbf{M}]_{x}$, respectively. The $f_{i}$ is a filter and defined as follows:

$$
f_{i}=\sigma_{i} D_{i i}=\frac{\sigma_{i}^{2}}{\sigma_{i}^{2}+\alpha^{2}} \simeq \begin{cases}1, & \sigma_{i} \gg \alpha \\ \frac{\sigma_{i}^{2}}{\alpha^{2}}, & \sigma_{i} \ll \alpha\end{cases}
$$


Several approaches are introduced in the literature to find the optimal value of $\alpha$ such as the cross-validation approach [94], and the L-curve criterion [91, 95]. The L-curve method is here implemented. The L-curve refers to a log-log plot for the $\left\|\mathbf{B}_{\text {sdo }}\right\|_{2}$ versus the norm of the residual term $\left\|[\mathbf{M}]_{x} \mathbf{B}_{\text {sdo }}-\mathbf{T}\right\|_{2}$. The resulting curve usually takes the shape 'L', as shown in fig. 5.2. It is a convenient graphical tool for displaying the trade-off between the size of the regularization term and its fit to the given data as the regularization parameter $\alpha$ varies.

The mathematical derivation for the optimal value for the regularization parameter $\alpha$ in this type of problem can be found in reference [91]. Here we show graphically how to obtain this optimal value of $\alpha$. Consider the case of a spacecraft in a circular orbit of $750 \mathrm{~km}$. At one point in this orbit, the L-curve is shown in fig. 5.2. The vertical axis shows $\left\|\mathbf{B}_{\text {sdo }}\right\|_{2}$, and the horizontal axis shows $\left\|[\mathbf{M}]_{x} \mathbf{B}_{\text {sdo }}-\mathbf{T}\right\|_{2}$. As the value of $\alpha$ is increased from zero, the solution point moves on the vertical curve down, and the value of $\left\|\mathbf{B}_{\text {sdo }}\right\|_{2}$ is decreased until the corner point is reached. It is possible to think of the vertical curve as dominated mainly by the regularization term, since the residual term does not change significantly as the solution is moving on the vertical curve.

As we further increase the value of $\alpha$, the solution point starts to move on the horizontal curve, toward the right, increasing the value of the residual term. Referring to Eq. (5.4), one would search for the minimum of the residual term while maintaining 


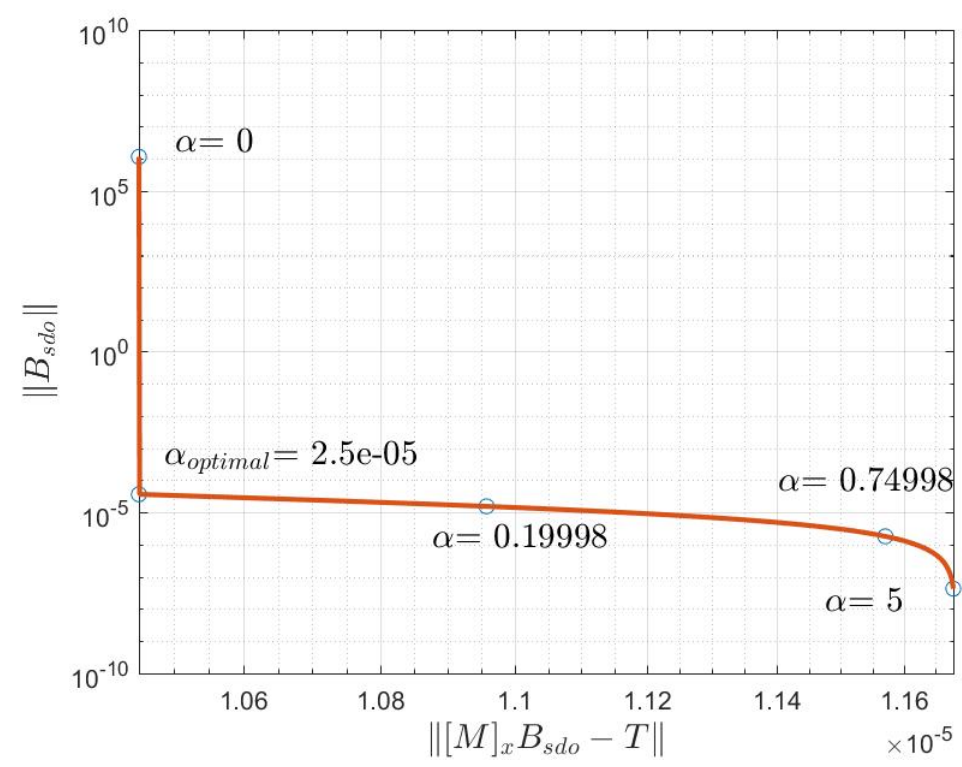

Figure 5.2: $\log -\log$ L-curve of the norm of the regularized term and the norm of residual term

as large as possible value for $\alpha$ so that the regularization term would be negligible. Hence, it can be concluded that the optimal value of $\alpha$, in fig. 5.2 , is the value that corresponds to the corner point of the L-curve, as it corresponds to the point of maximum $\alpha$ among all points that are near the minimum of the residual. This is the same solution obtained mathematically in reference [91].

The curvature of the L-curve plays an important role in understanding and using this criterion. The optimal regularization parameter $\alpha$, located at the corner of the $\log$-log L-curve, corresponds to the maximum curvature $\kappa$. The curvature $\kappa$ can be computed as follows [91]:

$$
\kappa=2 \frac{\eta \rho}{\eta^{\prime}} \frac{\alpha^{2} \eta^{\prime} \rho+2 \alpha \eta \rho+\alpha^{4} \eta \eta^{\prime}}{\left(\alpha^{2} \eta^{2}+\rho^{2}\right)^{\frac{3}{2}}}
$$


where

$$
\begin{aligned}
\eta & =\left\|\mathbf{B}_{\text {sdo }}\right\|_{2}^{2} \\
\eta^{\prime} & =-\frac{4}{\alpha} \sum_{i=1}^{q}\left(1-f_{i}\right) f_{i}^{2} \frac{\left(u_{i}^{T} \mathbf{T}\right)^{2}}{\sigma_{i}^{2}}
\end{aligned}
$$

The curvature $\kappa$ is a function of $\left\|\mathbf{B}_{\text {sdo }}\right\|_{2}$, the residual, and regularization parameter $\alpha$. Since the optimal $\alpha$ corresponds to the maximum $\kappa$, then finding the optimal $\alpha$ is a one-dimensional maximization problem of the curvature $\kappa$ given in Eq. (5.10). This one-dimensional optimization can be carried out using a golden section optimization approach, or through a numerical exhaustive search for a range of $\alpha$. This completes the process of computing the magnetic field pseudo measurement vector $\mathbf{B}_{\text {sdo }}$. The following sections demonstrate the use of this method in spacecraft magnetic attitude control. The following section briefs the control logic used in this chapter.

\subsection{Control Law}

The control objective here is to drive the spacecraft body frame $b$ to align with the

inertial frame $i$. Therefore, from Eq. (2.2), it follows that $[R(\mathbf{q})]=\mathbf{1}_{3 x 3}$. Thus the 
objective is to design control law so that $\boldsymbol{\omega} \rightarrow 0$ and $\mathbf{q}_{v} \rightarrow 0$.

Here, the proportional derivative-like control logic presented in reference [48] as a modification of those in [7, 11], is adopted. The process of computing the control torque starts with computing the designed torque $\mathbf{T}_{r e q}$ as follows:

$$
\mathbf{T}_{r e q}=-\left(\zeta^{2} k_{p} \mathbf{q}_{v}+\zeta k_{d} \boldsymbol{\omega}\right)
$$

where $k_{p}>0$ is the proportional gain, $k_{d}>0$ is the derivative gain and $\zeta$ is a parameter introduced to limit the control torque to ensure the controllability [66]. The control limit parameter is bounded: $0<\zeta<\zeta^{*}$. This control ensures that the equilibrium point is locally exponentially stable, when neglecting the duty cycle effect, as shown in [10].

In this work, we will take into account the duty cycle effect in studying the system stability. By using the magnetic field pseudo measurement vector $\mathbf{B}_{\text {sdo }}$ at the cycles where the proposed control scheme will not use real magnetometer measurement, the required dipole moment is calculated as follows:

$$
\mathbf{M}=\bar{f} \frac{\mathbf{B}_{s d o} \times \mathbf{T}_{r e q}}{\left\|\mathbf{B}_{s d o}\right\|^{2}}
$$


where $\bar{f}$ is defined as follows:

$$
\bar{f}\left(t, \epsilon T_{s}, \bar{\delta}\right)= \begin{cases}1 & 0 \leq \operatorname{Mod}\left(t, \epsilon T_{s}\right) \leq \bar{\delta} \epsilon T_{s} \\ 0 & \bar{\delta} \epsilon T_{s} \leq \operatorname{Mod}\left(t, \epsilon T_{s}\right) \leq \epsilon T_{s}\end{cases}
$$

The function $\bar{f}\left(t, \epsilon T_{s}, \bar{\delta}\right)$ can be described in a compact form as follows:

$$
\bar{f}\left(t, \epsilon T_{s}, \bar{\delta}\right)=1+\frac{\operatorname{Mod}\left(\operatorname{Mod}\left(t, \epsilon T_{s}\right), \bar{\delta} \epsilon T_{s}\right)-\operatorname{Mod}\left(t, \epsilon T_{s}\right)}{\bar{\delta} \epsilon T_{s}},
$$

in which its average will be $\bar{f}_{\text {avg }}=\bar{\delta}$. It is clear here that $\bar{f}_{\text {avg }}>f_{\text {avg }}$ for $\epsilon>1$ and for $\epsilon=\infty$, the value of $\bar{f}_{\text {avg }}=1$. Combining Eq. (5.14) and Eq. (2.7), the applied torque to the spacecraft is:

$$
\mathbf{T}=-\bar{f} \mathbf{B} \times \frac{\mathbf{B}_{s d o} \times \mathbf{T}_{r e q}}{\left\|\mathbf{B}_{s d o}\right\|^{2}}=\bar{f}\left[\Gamma_{s d o}(t)\right] \frac{\mathbf{T}_{r e q}}{\left\|\mathbf{B}_{s d o}\right\|^{2}},
$$

where the matrix $\left[\Gamma_{s d o}(t)\right]=[\mathbf{B}]_{x}\left[\mathbf{B}_{s d o}\right]_{x}^{T}$ is positive semi-definite assuming $\mathbf{B} \approx \mathbf{B}_{s d o}$. In this case, the computed magnetic field pseudo measurement vector $\mathbf{B}_{\text {sdo }}$ is used for computing the required dipole moment. The spacecraft applied torque is generated via the interaction between this generated dipole moment and actual ambient magnetic field $\mathbf{B}$. 


\subsubsection{Stability Analysis}

This section is dedicated for the stability analysis of the three-axis attitude regulation of an inertial pointing spacecraft, using the control law in Eq. (5.13). Here the effect of intermittent actuation of the magnetic rods is accounted for through the function $f$, unlike most of the existing work in the literature. Therefore, during this analysis, B, $f$ and $[\Gamma]$ will be used instead of $B_{s d o}, \bar{f}$ and $\left[\Gamma_{s d o}\right]$ in Eqs. (5.14) and (5.17). Later, the effect of the proposed control algorithm on the system stability will be demonstrated.

To check the stability of this control law for inertial pointing maneuver, the following Lyapunov function is introduced:

$$
V\left(\boldsymbol{\omega}, \mathbf{q}_{v}\right)=\frac{1}{2} \boldsymbol{\omega}^{T}[I] \boldsymbol{\omega}+2 K_{P}\left(1-q_{0}\right)
$$

where $K_{p}$ is positive definite. The Lyapunov function $V(0,0)=0$ and $V\left(\boldsymbol{\omega}, \mathbf{q}_{v}\right)$ is positive definite elsewhere. The average theory will be employed in order to convert the time-variant system into a time-invariant one 85, given the condition in Eq. (3.14) [10, 42, 57]. The derivative of the Lyapunov function (5.18), using Eqs. (2.1), 2.5), 
(3.14), 5.13), and 5.17) will be as follows:

$$
\dot{V}\left(\boldsymbol{\omega}, \mathbf{q}_{v}\right)=-f \zeta k_{d} \boldsymbol{\omega}^{T}\left[\Gamma_{a v g}\right] \boldsymbol{\omega}-f \zeta^{2} k_{p} \boldsymbol{\omega}^{T}\left[\Gamma_{a v g}\right] \mathbf{q}_{v}+K_{P} \boldsymbol{\omega}^{T} \mathbf{q}_{v}+\boldsymbol{\omega}^{T} \mathbf{T}_{D}
$$

Remark 5.1: The $\dot{V}$ is not negative semi definite all the time. This is true even if we try to select the controller parameters, $k_{p}, k_{d}$ and $\zeta$, so as to guarantee that the first term in the right hand side of Eq. 5.19 is the dominate term. This is attributed to the fact that when $f=0$, during the desaturation and measurement period, there is no control authority over the spacecraft. Hence, this system can not be stable in the sense of Lyapunov stability theory.

The closed loop dynamics by substituting Eqs. (3.14), (5.13) and (5.17) into Eq. (2.5) will be:

$$
[I] \dot{\boldsymbol{\omega}}+f \zeta k_{d}\left[\Gamma_{a v g}\right] \boldsymbol{\omega}+f \zeta^{2} k_{p}\left[\Gamma_{a v g}\right] \mathbf{q}_{v}=\mathbf{T}_{D}
$$

where the gyroscopic coupling term $\boldsymbol{\omega} \times[I] \boldsymbol{\omega}$ is neglected as it is usually small and being of second order term for $\|\omega\| \ll 1$ [1]. Taking the derivative of Eq.(5.20] with the use of Eq.(2.1), we obtain a second-order differential equation in terms of $\boldsymbol{\omega}$ as follows:

$$
[I] \ddot{\boldsymbol{\omega}}+f \zeta k_{d}\left[\Gamma_{a v g}\right] \dot{\boldsymbol{\omega}}+f \zeta^{2} k_{p}\left[\Gamma_{a v g}\right]\left[q_{0}\left[\mathbf{1}_{3 x 3}\right]+\left[\mathbf{q}_{v}\right]_{x}\right] \boldsymbol{\omega}=\dot{\mathbf{T}}_{D}
$$


Let the symmetric positive definite matrix $\left[\Gamma_{a v g}\right]=[A]$ and the skew symmetric $\operatorname{matrix}\left[\mathbf{q}_{v}\right]_{x}=[B]$, then

$$
[A][B]=\operatorname{tr}\left([A]^{T}[B]\right)=A_{i j} B_{i j}=-A_{j i} B_{j i}=-[A][B] \Rightarrow 2[A][B]=0 \Rightarrow[A][B]=0
$$

Therefore, $\left[\Gamma_{\text {avg }}\right]\left[q_{0}\left[\mathbf{1}_{3 x 3}\right]+\left[\mathbf{q}_{v}\right]_{x}\right]=q_{0}\left[\Gamma_{\text {avg }}\right]$ is positive definite matrix. It is assumed that the control law will always take the short rotation. In other words, the quaternion will be switched to the shadow set in case of $q_{0}<0$. Hence, $q_{0}$ is always positive. For slow variation or steady total disturbance torque assumption, $\dot{\mathbf{T}}_{D} \approx 0$, Eq. 5.21 represents a stable spring mass damper system with positive definite matrices multiplied by $\ddot{\boldsymbol{\omega}}, \dot{\boldsymbol{\omega}}$ and $\boldsymbol{\omega}$. By replacing the function $f$ with its average $f_{\text {avg }}=\delta$ and taking the limit of Eq. (5.21), we get the steady sate condition

$$
\delta \zeta^{2} k_{p}\left[\Gamma_{a v g}\right]\left[q_{0(s s)}\left[\mathbf{1}_{3 x 3}\right]+\left[\mathbf{q}_{v(s s)}\right]_{x}\right] \boldsymbol{\omega}_{s s}=0
$$

where $\boldsymbol{\omega}_{s s}, q_{0(s s)}$ and $\mathbf{q}_{v(s s)}$ are the steady state values of the spacecraft angular velocity and quaternion components. With $\delta>0, k_{p}>0, \zeta^{2}>0,\left[\Gamma_{a v g}\right]$ is positive definite matrix [10, 42, 57], $\left[q_{0(s s)}\left[\mathbf{1}_{3 x 3}\right]+\left[\mathbf{q}_{v(s s)}\right]_{x}\right] \rightarrow\left[\mathbf{1}_{3 x 3}\right]$ for $t \rightarrow \infty$, the steady state angular velocity error $\boldsymbol{\omega}_{s s}=0$. Thus, even in the presence of the disturbance torque $\mathbf{T}_{D}$, the angular velocity error will decay to zero asymptomatically. However, this is not the case with the attitude error. Taking the limit of Eq. (5.20), the steady 
state error in terms of the quaternion vector part will be :

$$
\mathbf{q}_{v(s s)}=\left[\Gamma_{a v g}\right]^{-1} \frac{\mathbf{T}_{D}}{\delta \zeta^{2} k_{p}}
$$

Remark 5.2: It is clear from Eq. (5.24), with bounded disturbance torque assumption, that the attitude error will settle on a finite offset and will be bounded in the presence of $\mathbf{T}_{D}$ and $\delta$. Therefore, the control law Eq. (5.13) is stabilizing in the sense of Lagrange stability theory.

Note: It is worth noting that asymptomatic stability may be fulfilled by adding an integral feedback term to this state feedback control law Eq. (5.13). However, this is kept as a future work.

It can be concluded from the above stability analysis that there is another advantage of increasing the magnetic rods duty cycle using the proposed control algorithm: the steady state attitude error for the system in Eq. (5.24) is decreased, in addition to reducing the maneuver time and power consumption. This will be verified using numerical simulations in Section 5.4. 


\subsubsection{Gain selection Criteria Discussion}

The control law 5.13 is able to stabilize the spacecraft with specific relation between the control gains. In which the proportional gain is smaller than the derivative one. This proofed through bifurcation analysis and numerical continuation of solutions on large control values [66]. Control gains selection criteria is crucial for the success of the control. Furthermore, it has been reported that the low angular velocity is crucial for the controllability using the averaging technique [96]. The Gurwin satellite is an example of three axis control failure because of the controller design. However, there are other successful cases such as Oersted satellite and TANGO satellite [66].

There are several studies provided guidelines to find the gains $k_{p}, k_{d}$, and $\zeta$ in [7, 10, 11]. Lovera et al. described a try and error approach to find such gains [76]. By First selecting a positive value for each of the gains $k_{p}$ and $k_{d}$, then reducing the $\zeta$ value until getting a steady sate response via simulation. Then the gains $k_{p}$ and $k_{d}$ are adjusted to get the required system response. The try and error approach will continue until a sufficient performance is fulfilled. Damaren [97] suggested a constrained condition to find the gains $k_{p}$ and $k_{d}$ by augmenting the control system with independent three axis actuation scheme. In which the three-axis attitude stabilization will be achieved via hybrid actuation from magnetic rods and reaction wheels. Jinsong et al. 98] formulated an optimization problem to find the gains $k_{p}$ and $k_{d}$ in which 
the performance index is a function in quaternion and angular velocity error. The problem treated as fixed time by using a scaling factor. In which, the scale factor is to be optimized and included in the performance index. However, Fabio [99] formulated a different derivative free optimization problem to find these gains. The performance index was the settling time. The settling time is a discontinuous function in initial condition and there are infinite number of initial conditions, this optimization problem presented as minimum maximum derivative free problem. As the gradient-based optimization methods are not applicable to the discontinuity problem. After using initial gains' values using the try and error approach, there is an internal optimization loop to find the maximum (worst) settling time with the different initial conditions. Another external optimization loop to find the gains related to the worst settling time. This optimized approach found the optimal gains at the worst settling time. However, by changing the initial conditions, the gains optimally is lost. There is also a proposed simple scheme to estimate an initial values of the gain based on analytical results and Floquet theory in [24, 48, 63, 100, 101]. Then the try and error approach is used to get the required performance. In [102] the feedback gain is improved with errors in the inertia tensor. However, uncontrollable situation can be fulfilled with errors in attitude determination and disturbances [6]. In the light of the above cited works and the survey in [66], Most of the results on the control gains selection are valid for the satellite with commensurable moments of inertia and a try and error approach is required at some point. Here the try and error approach is followed. 


\subsection{Numerical Simulation Results}

This section is dedicated to verify the stability analysis results in section 5.3.1. In which the duty cycle effect for inertial pointing three-axis attitude control is considered. Then the next part will be dedicated to assess the performance of the proposed scheme to extend the activation of the magnetic rods (duty cycle).

\subsubsection{Inertial Pointing Three-Axis Attitude Control}

The goal of this section is to highlight the impact of the disturbance torques and the duty cycle effect on the steady state error for inertial pointing three-axis control maneuvers. The hardware configuration and spacecraft parameters are similar to those in reference [62]. Table 5.1 shows both the spacecraft and orbital parameters.

Table 5.1

Spacecraft and orbital parameters

\begin{tabular}{lc}
\hline \hline Parameter & Value [unit], [uncertainty $]$ \\
\hline$\left[I_{x}, I_{y}, I_{z}\right]^{T}$ & {$[0.196,0.202,0.202]^{T}\left[\mathrm{~kg} \cdot \mathrm{m}^{2}\right],[10 \%]$} \\
Max. Dipole Moment & $\pm 1.83\left[\mathrm{Am}^{2}\right]$ \\
Altitude & $639.212[\mathrm{~km}]$ \\
Inclination & $97.868^{\circ}$ \\
Right ascension of the ascending & $157.305^{\circ}$ \\
True anomaly at initial time & $277.29^{\circ}$ \\
Eccentricity & 0 \\
\hline
\end{tabular}




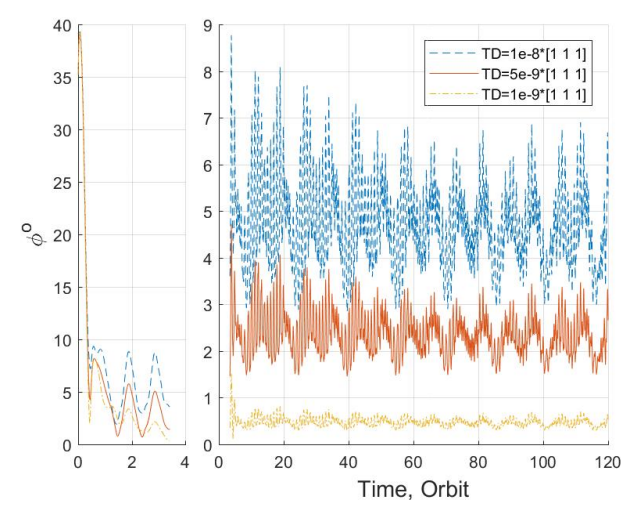

Figure 5.3: Spacecraft attitude error history in terms of principal rotation error for different constant $\mathbf{T}_{D}$.

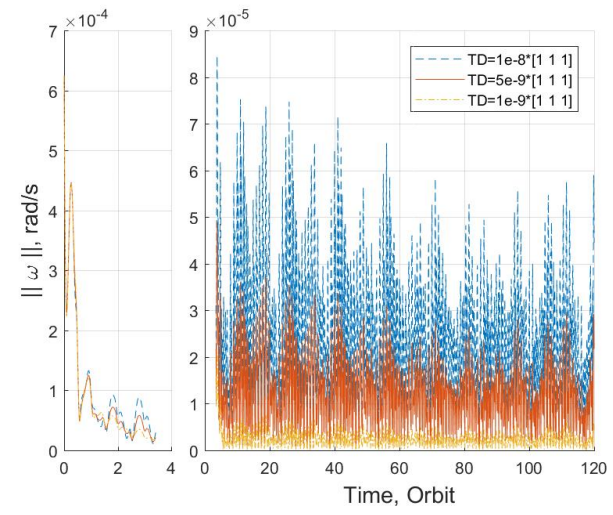

Figure 5.4: Spacecraft angular velocity magnitude history for different constant $\mathbf{T}_{D}$.

Here the initial spacecraft attitude and angular velocity are selected randomly. The maneuver objective is to align the spacecraft body frame with the inertial frame. In this section, to make this presentation more clear, the attitude error will be represented in terms of the principal rotation error angle $\phi$ between the current attitude and the desired one (desired attitude is $\mathbf{q}_{v} \rightarrow 0$ for regulation) for long maneuver (120 orbits). Different cases are established to check the effect on the attitude steady state error according to Eq. (5.24).

For the first case, three different fixed disturbance torque vectors, $\mathbf{T}_{D}=1 e^{-8} *\left[\begin{array}{lll}1 & 1 & 1\end{array}\right]$, $\mathbf{T}_{D}=5 e^{-9} *\left[\begin{array}{lll}1 & 1 & 1\end{array}\right]$ and $\mathbf{T}_{D}=1 e^{-9} *\left[\begin{array}{lll}1 & 1 & 1\end{array}\right]$ are set with $\xi^{2} k_{p}=1 e^{-6}, \xi k_{d}=4 e^{-4}$ and $\delta=0.7$. Figure 5.3 shows the attitude error history in terms of the principal rotation angle. As can be seen from fig 5.3 that the averaged values of the steady state principal rotation angle are $\phi_{\text {avg }(s s)}=4.7281^{\circ}, \phi_{\text {avg }(s s)}=2.3616^{\circ}$ and $\phi_{\text {avg }(s s)}=$ $0.4745^{\circ}$, respectively. It is clear that the attitude error change is proportional to the 


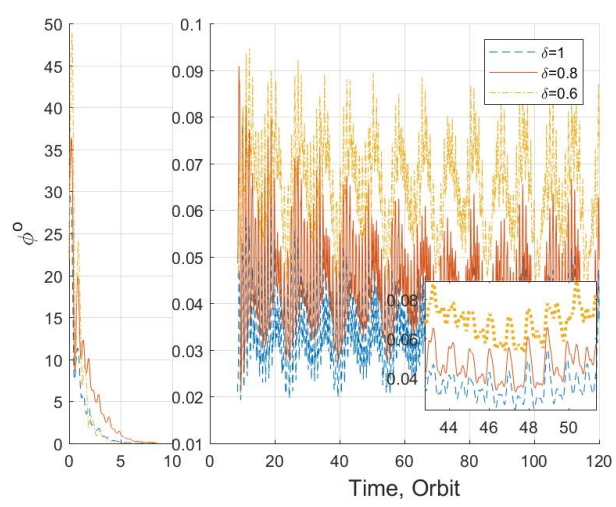

Figure 5.5: Spacecraft attitude error history in terms of principal rotation error for different duty cycles.

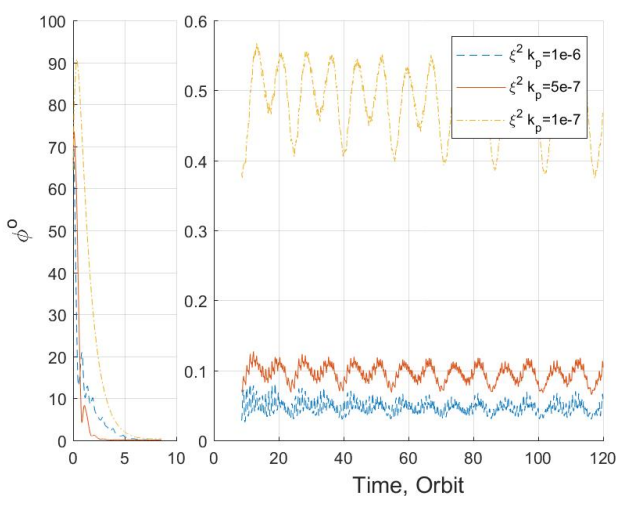

Figure 5.6: Spacecraft attitude error history in terms of principal rotation error for different proportional gain $k_{p}$.

change in $\mathbf{T}_{D}$, in agreement with the above analysis (Eq. (5.24)). The oscillation of the steady state error is attributed to the fact that system is time-variant due to the changing the magnetic field around the spacecraft.

Figure 5.4 renders the angular velocity magnitude history. The continuous oscillation is attributed to the fact that controller attempts continuously to reduce the attitude error. However, the angular velocity is settled to small values, close to zero, as expected from the stability analysis. Therefore, the remaining results in this section will focus only on the attitude error.

In the second case, the duty cycle effect is assessed by changing the duty cycle percent: $\delta=0.6, \delta=0.8$ and $\delta=1$, with $\xi^{2} k_{p}=1 e^{-6}, \xi k_{d}=4 e^{-4}$ and $\mathbf{T}_{D}=1 e^{-8} *\left[\begin{array}{lll}1 & 1 & 1\end{array}\right]$. The average values of the steady state principal rotation angle are $\phi_{\text {avg(ss) }}=0.0654^{\circ}$, $\phi_{\text {avg(ss) }}=0.0444^{\circ}$ and $\phi_{\text {avg(ss) }}=0.0335^{\circ}$, respectively. Figure. 5.5 shows that the attitude error is changed by almost the same change factor in $\delta$, in agreement with 
Eq. (5.24).

For the third case, the proportional gains are selected to be $\xi^{2} k_{p}=1 e^{-7}, \xi^{2} k_{p}=5 e^{-7}$

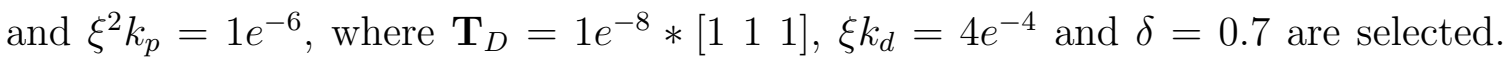
The average values of the steady state principal rotation angle are $\phi_{\text {avg }(s s)}=0.4809^{\circ}$, $\phi_{\text {avg }(s s)}=0.0953^{\circ}$ and $\phi_{\text {avg }(s s)}=0.0472^{\circ}$, respectively, see fig.5.6. The steady state error percent change is consistent with the change in $\xi^{2} k_{p}$.

Overall, the magnetic field desaturation and magnetometer measurement period is a manufacturer specification related to the design of the magnetic rods [62]. This period degrades, in general, the magnetic ACS and should be considered during the design phase of the ACS. Decreasing such a period, or equivalently increasing the duty cycle percentage of the magnetic rods, will decrease the attitude steady state error, as a first benefit, as shown on the above analysis and results. That leads us to check if the proposed scheme is able carry out attitude maneuver, which is done in the next section. 


\subsubsection{Proposed Control Scheme for Extending the Duty Cy- cle}

In this section, the performance of the proposed algorithm (upper part of fig. 5.1) is demonstrated via Monte Carlo simulation runs, and is compared to the "reference" case in which both the magnetic rods and magnetometers are turned on, alternately, during one cycle period (lower part of fig. 5.1). To highlight the impact of the proposed algorithm compared to the reference one, the output results from the proposed algorithm are normalized by the results from the reference algorithm.

Same disturbance and sensor noises configuration are set as discussed in section 3.3 . Table 5.2 lists the required parameters to compute the disturbance torques.

Table 5.2

Disturbance Parameters

\begin{tabular}{|c|c|c|}
\hline Parameter & Value [unit] & Uncertainty \\
\hline$\rho^{*}$ & $2.01 .10^{-14}\left[K g \cdot m^{3}\right]$ & - \\
\hline$R_{m p}$ & {$\left[\begin{array}{lll}9 & 11 & 12\end{array}\right][\mathrm{mm}]$} & $10 \%$ \\
\hline$C_{D}$ & 2 & - \\
\hline Spacecraft dimension & {$\left[\begin{array}{lll}23 & 23 & 29\end{array}\right] \quad[\mathrm{cm}]$} & - \\
\hline$\left\|\mathbf{M}_{r d s}\right\|$ & $1 e^{-4}\left[A m^{2}\right]$ & $10 \%$ \\
\hline$C_{r k}$ & 1.5 & - \\
\hline$F_{\text {solar }}$ & $1366\left[w / m^{2}\right]$ & - \\
\hline
\end{tabular}

The parameters of the control algorithms are as follows: $T_{s}=0.1[s]$ and $\delta=0.7$. 
Therefore, the control command frequency is $f_{c}=1 / 0.1$, and the gyroscope measurements frequency is selected to be $f_{\omega}=5 / 0.1$. The control gains are: $\xi=0.001$, $k_{p}=1$, and $k_{d}=0.4$. A 30-minute confirmation window is used after the spacecraft is settled to the desired attitude in order to assure that the spacecraft reached and settled near the desired attitude.

\section{Case study}

Before presenting the statistical Monte Carlo analysis, the results from a sample run for the proposed algorithm are presented. In this example, the satellite initial angular velocity and initial attitude are selected randomly along with random desired attitude. The simulation runs for 12 orbits to check the performance of the proposed algorithm. Figure 5.7 depicts the time behavior of the satellite's angular velocity of the body frame w.r.t the inertial frame for the reference case, labeled (Ref), along with the time behavior of the satellite's angular velocity for the proposed algorithm with $\epsilon=3$, labeled (Proposed 3(Ts)). The proposed algorithm is able to preserve almost constant angular velocity vector after settling the spacecraft to the desired attitude, as shown in fig 5.7. The attitude history, quaternion, is shown in fig 5.8 . where the proposed algorithm is able to settle at the desired attitude; the reference algorithm also settles at the desired attitude. In fig. 5.8, a range of $5 \%$ around each quaternion component is plotted on the zoom-in figure on the right, where it can be 

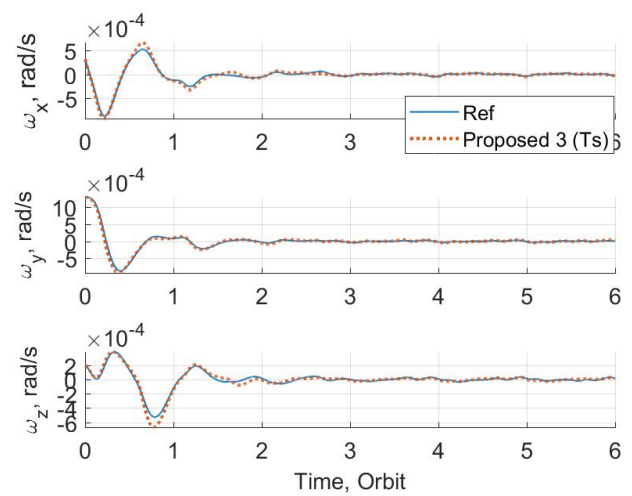

Figure 5.7: Spacecraft angular velocity history.
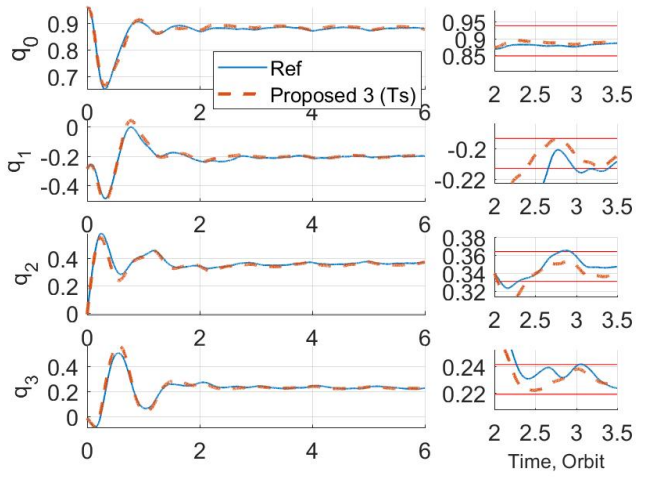

Figure 5.8: Spacecraft quaternion history over time (orbits).

seen that performance of the proposed algorithm outperforms the reference algorithm in terms of settling sooner with the error bounds. More on the comparison between the two algorithms is discussed next.

The above simulation is repeated for several $\epsilon$ values of the proposed algorithm. In this section, two parameters are used for comparison; the mean value of the total activation time $\mathbf{t}_{\text {on }}$ of the three magnetic rods, computed using Eq. (1.1), as an indication of the power consumption, and the settling time (as indication of the maneuver time) to bring the spacecraft attitude to within 5\% error of the desired attitude. Both parameters are in the normalized form w.r.t the same parameters from the reference case algorithm.

Figure 5.9 depicts the normalized maneuver time for the proposed algorithm for different values of $\epsilon$. There is a reduction in the maneuver time when using the proposed algorithm; this reduction time increases as $\epsilon$ increases, up to a point. In 


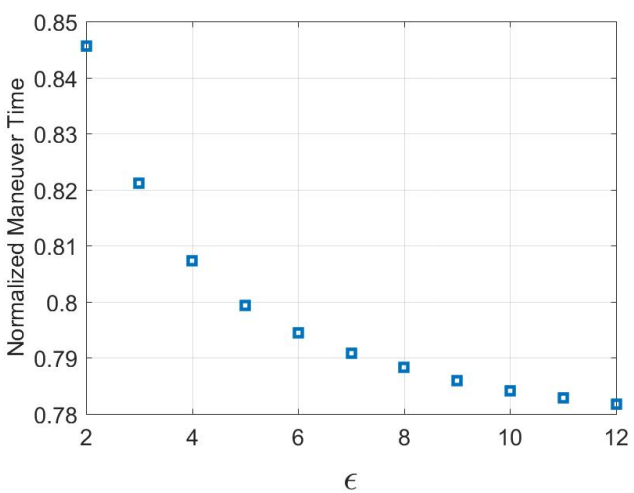

Figure 5.9: The $\epsilon$ parameter effect on the normalized maneuver time

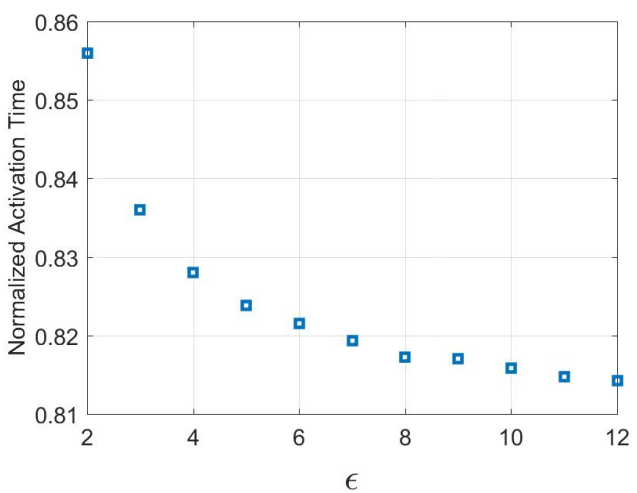

Figure 5.10: The $\epsilon$ parameter effect on the normalized $\mathbf{t}_{\text {on }}$

this case study, a $15 \%$ to $21 \%$ saving in the maneuver time can be achieved.

As a measure for the consumed power by the magnetic rods, fig 5.10 depicts the mean of the normalized values of the magnetic rods activation times $\mathbf{t}_{\text {on }}$, for the proposed algorithm. The magnetic rods activation time reduces as $\epsilon$ increases. The improvement in power consumption decays as $\epsilon$ goes beyond the value of 8 . A savings of $18 \%$ in the power consumption of the magnetic rods can be achieved in this case.

For further assessment of the proposed algorithm, three parameters are introduced. The first parameter is the correlation coefficient (CC) between the computed magnetic field pseudo measurement, $\mathbf{B}_{\text {sdo }}$, and its true value, $\mathbf{B}$. When the $\mathrm{CC}$ is close to 1 , it indicates strong correlation. The correlation is weak when the $\mathrm{CC}$ becomes close to 0. The CC is computed as shown in Eq. (5.25).

$$
C C=\frac{\sum_{i=1}^{n}\left(\mathbf{x}_{i}-\overline{\mathbf{x}}\right)\left(\mathbf{y}_{i}-\overline{\mathbf{y}}\right)}{\sqrt{\sum_{i=1}^{n}\left(\mathbf{x}_{i}-\overline{\mathbf{x}}\right)^{2} \sum_{i=1}^{n}\left(\mathbf{y}_{i}-\overline{\mathbf{y}}\right)^{2}}}
$$


where $n$ is the number of sample elements, $\mathbf{x}_{i}$ and $\mathbf{y}_{i}$ represent the true and the computed equivalent values, respectively, and $\overline{\mathbf{x}}$ and $\overline{\mathbf{y}}$ represent the mean of the true and the computed values, respectively.

Figure 5.11 depicts the $\mathrm{CC}$ of the computed magnetic field pseudo measurement, for the proposed algorithm, versus the change in $\epsilon$ values. As $\epsilon$ increases the correlation becomes weak. This is expected, since increasing $\epsilon$ means using magnetometers data less frequent, and hence the computed magnetic field drifts away from the true one. It is observed, however, that at small $\epsilon$ values $(\epsilon=2$ and $\epsilon=3)$ the computed magnetic field pseudo measurement is strongly correlated with the true one.

A second parameter that measures the quality of the computed magnetic field pseudo measurement is the scatter index (SI) which indicates statistically how the computed quantity is scattered around the true one. The SI is computed as follows:

$$
S I=\sqrt{\frac{\sum_{i=1}^{n}\left(\left(\mathbf{x}_{i}-\overline{\mathbf{x}}\right)\left(\mathbf{y}_{i}-\overline{\mathbf{y}}\right)\right)^{2}}{\sum_{i=1}^{n} \mathbf{x}_{i}^{2}}}
$$

Figure 5.12 depicts the SI for the proposed algorithm versus the change in $\epsilon$ values. As shown in fig. 5.12, smaller $\epsilon$ values have less SI. Finally, the Normalized Root Mean Square Error (NRMSE) can be used to assess the goodness of the computed magnetic field pseudo measurement. Figure 5.13 depicts the NRMSE for the proposed algorithm versus the change in $\epsilon$. It can be concluded from the three parameters above 


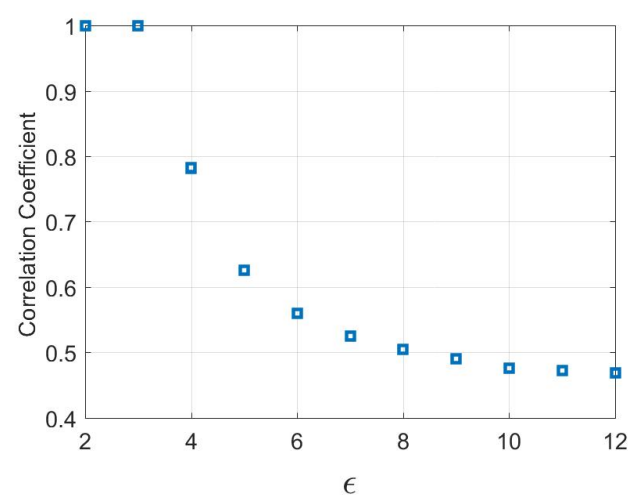

Figure 5.11: The $\epsilon$ parameter effect on the CC of the computed $\mathbf{B}_{\text {sdo }}$.

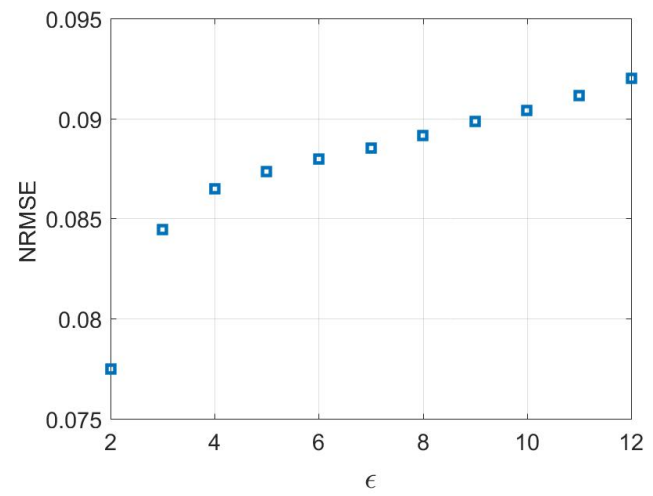

Figure 5.13: The $\epsilon$ parameter effect on the NRMSE of the computed $\mathbf{B}_{s d o}$.

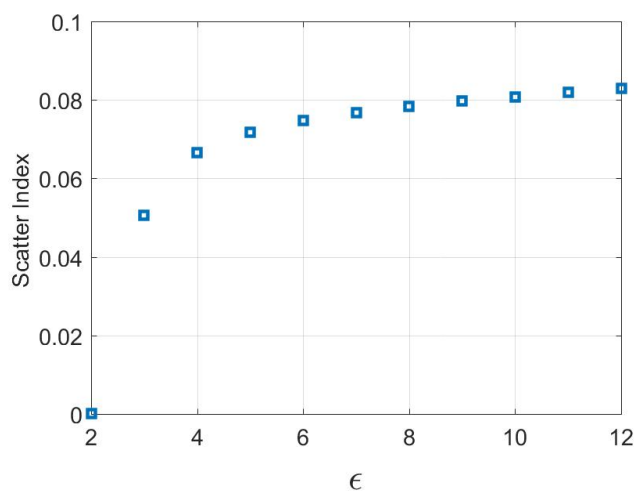

Figure 5.12: The $\epsilon$ parameter effect on the SI of the computed $\mathbf{B}_{s d o}$.

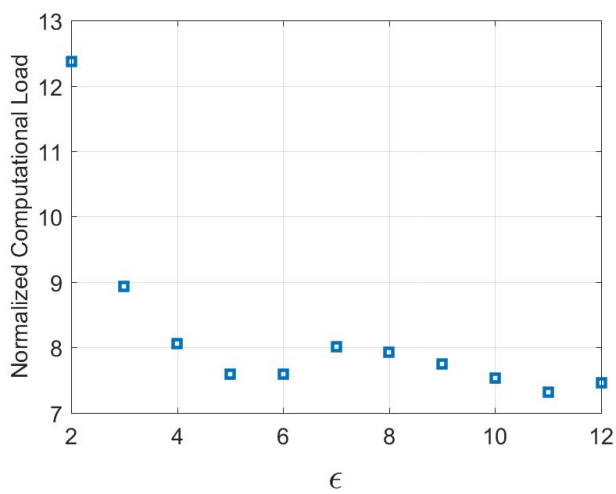

Figure 5.14: The $\epsilon$ parameter effect on the normalized computational load

that lower the $\epsilon$ value, the more accurate is $\mathbf{B}_{s d o}$; moreover these curves can be used to select a suitable value for $\epsilon$ depending on the desired accuracy and performance. It is also observed that no further improvement can be achieved for values of $\epsilon$ beyond 8 in terms of maneuvering and activation times. This is expected since when $\epsilon=8$, the proposed duty cycle becomes $\bar{\delta}=0.9625$, in this case study, while at $\epsilon=12$, $\bar{\delta}=0.975$. Therefore, there is no significant improvement in $\bar{\delta}$ can be achieved when increasing $\epsilon$ beyond 8 . 
Regarding the computational cost of the proposed algorithm, fig. 5.14 shows the variation of the computational load versus $\epsilon$, normalized relative to the computational load of the reference algorithm. The computational load is defined as the total required computational cost needed throughout the maneuver, i.e. until the spacecraft settles to the desired attitude. It is not the computational load per duty cycle. Clearly, the proposed algorithm is significantly higher than the reference algorithm in terms of the computational load. This can be explained since the proposed $\mathbf{B}_{s d o}$ computations involve in-line optimization, and eigenvalues and eigenvectors calculations. Since the maneuver time is reduced by increasing $\epsilon$, the total number of carrying out the proposed algorithm will be reduced. This Leads to, in general, reducing the total computational cost as $\epsilon$ increases, as shown in fig 5.14 . It is noted here that the results obtained in fig. 5.14 are generated based calculations carried out using the Matlab software, which is slow in general. An executable code would result in a significant improvement in terms of the computational time for the proposed algorithm, while not significantly affecting the computational time of the reference algorithm since there is not much computations in the reference algorithm. As a result, it is expected that the normalized computational time in fig. 5.14 can be reduced. In the following Monte Carlo analysis, the $\epsilon$ value is selected to be 3 . 


\section{Monte Carlo simulation analysis}

Results are here presented for 4,800 Monte Carlo simulation runs; 2, 400 for the reference algorithm and 2, 400 for the proposed one. The 2, 400 runs is categorized into 240 different categories. Each category has its initial angular rates, initial quaternion, and required attitude. These values are generated randomly and are the same for both algorithms. So, each selection of the initial conditions and desired attitude is simulated 10 times, and the results are reported for both the activation time and the maneuver time (averaged over the 10 runs then normalized.) As a measure of the estimation error, the SI and NRMSE are computed and averaged.

The results of the simulation runs are here discussed. Figure 5.15 depicts the histogram and the Gaussian fit of the improvement in maneuver time for the 240 categories. Figure 5.15 shows that the maneuver time of the proposed algorithm is significantly less than that of the reference case. As shown in fig. 5.15, in some cases the proposed algorithm achieves a reduction in the maneuver time of about $80 \%$, whereas in other cases there is almost no improvement in terms of the maneuver time, compared to the reference algorithm. The mean value of the saving in terms of the maneuver time is $27.25 \%$, with $20.4 \%$ standard deviation.

The second comparison parameter is the activation time for the magnetic rods, which is considered a measure for the power consumption. Figure 5.16 depicts the histogram 


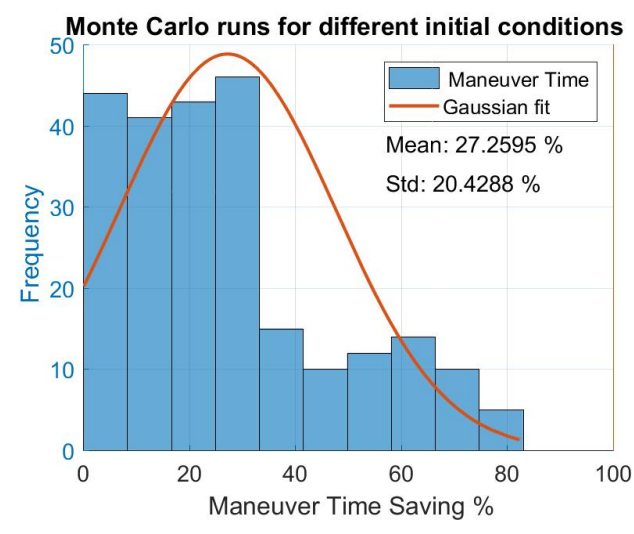

Figure 5.15: Maneuver time reduction percentage for 2,400 Monte Carlo runs for 240 different initial conditions.

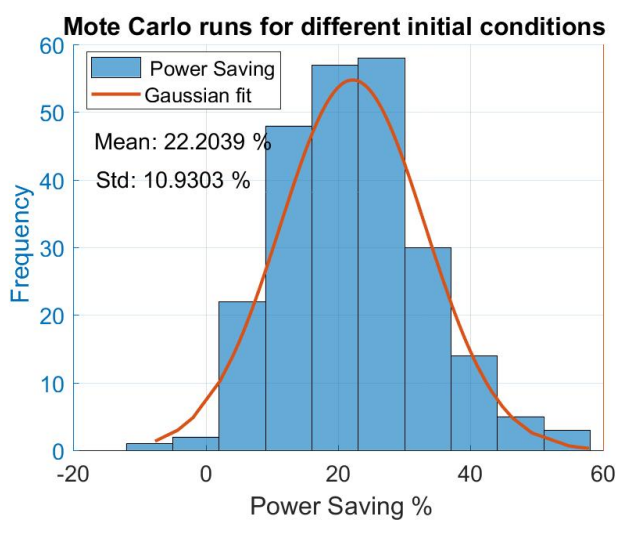

Figure 5.16: Power saving percentage for 2,400 Monte Carlo runs for 240 different initial conditions.

and the Gaussian fit of the improvement in mean of the total activation time of the magnetic rods for the 240 categories. The savings in terms of the activation time, using the proposed algorithm, goes up to about $55 \%$ in some cases. It is also observed that in three categories, the proposed algorithm required more activation time compared to the reference algorithm. In the other 237 categories the proposed algorithm requires less activation time. It is also noted that in the three categories where the proposed algorithms require more activation time, the proposed algorithm achieves the target attitude in less maneuver time. The mean value for the power saving using the proposed algorithm is $22.2 \%$, with a $10.9 \%$ standard deviation.

Finally, regarding the computed $\mathbf{B}_{\text {sdo }}$ vector accuracy in the above Monte Carlo simulations, the SI and the NRMSE parameters are computed. For the SI parameter, each one run resulted in a time history for $\mathbf{B}_{\text {sdo }}$ and $\mathbf{B}$ vectors. The SI is computed for each component of $\mathbf{B}_{\text {sdo }}$ resulting in $S I_{x}, S I_{y}$, and $S I_{z}$. An average for the three 


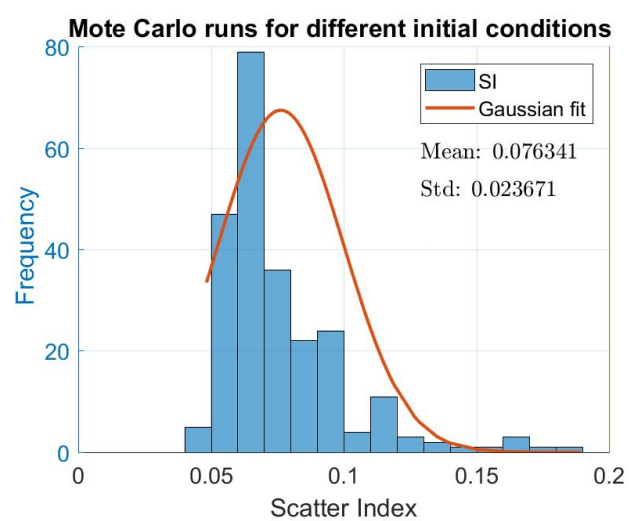

Figure 5.17: Scatter Index for 2,400 Monte Carlo runs for 240 different initial conditions.

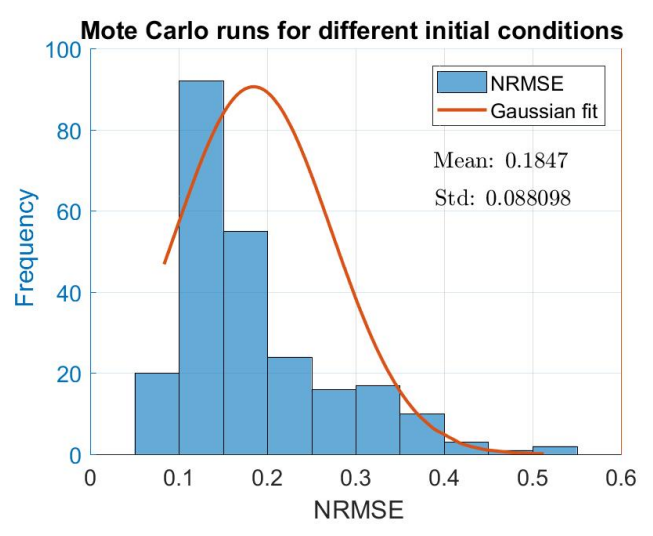

Figure 5.18: The NRMSE for 2,400 Monte Carlo runs for 240 different initial conditions.

SI values is then computed for each run. For each category, an average SI is computed for all runs in that category. Figure. 5.17 shows the obtained average values of the SI. As can be seen in fig. 5.17, the average SI ranges between 0.05 and about 0.18, which indicates good estimate of the $\mathbf{B}_{\text {sdo. }}$. The mean value of the SI of the 2,400 Monte Carlo simulations is 0.076 with a standard deviation of 0.02 .

For the NRMSE parameter, same process for SI is repeated here to get the average NRMSE for each category. Figure. 5.18 shows the obtained average values of the NRMSE. As can be seen in fig. 5.18, the average NRMSE ranges between 0.09 and about 0.51 . The mean value of the NRMSE of the 2, 400 Monte Carlo simulations is 0.1847 with a standard deviation of 0.0881 . 


\subsection{Verification of Magnetic Field Pseudo Mea- surement Computation Using Real Data}

This section is dedicated to verify the magnetic field pseudo measurement computation process that is presented in section 5.2 . The verification process will be conducted using real telemetry data from the CASSIOPE spacecraft. A brief overview over the CASSIOPE spacecraft hardware configuration, mission and the available telemetry data with its frequency is given in section 2.5 .

The following steps and assumption are made:

(1) The spacecraft angular velocities ${ }^{b} \omega_{b i}$ are given for the body frame with respect to the inertial frame using a gyroscope. The orbital angular velocity for elliptic orbit (the CASSIOPE orbital eccentricity $=0.0744616$ ) will be computed using the spacecraft position ${ }^{i} \mathbf{R}$ and velocity ${ }^{i} \mathbf{V}$ from the given Ephemeris as follows [103]:

$$
{ }^{i} \boldsymbol{\omega}_{o i}=\frac{{ }^{i} \mathbf{R} \times{ }^{i} \mathbf{V}}{\left\|^{i} \mathbf{R}\right\|^{2}}
$$

The CASSIOPE spacecraft is equipped with three star trackers to estimate the spacecraft attitude. The spacecraft attitude representation is given in the form of Euler 
angles that represent the spacecraft attitude with respect to the orbital frame resolved in the body frame. These Euler angles are transformed to Euler parameters, $\mathbf{q}_{t r u}$ quaternion and used for computing the transformation matrix to transform ${ }^{i} \boldsymbol{\omega}_{o i}$ to the body frame. Therefore, the quaternion can be propagated numerically using Eqs. (2.1), 2.4), and (5.27).

(2) The gyroscope measurements are prone to bias and noise, which is assumed here as a white noise. Both noise and bias can be handled online using the Extended Kalman Filter [104]. However, in this case, and for the short maneuver periods in hand, the bias will be handled first using batch optimization which finds the spacecraft initial angular velocity. The initial quaternion is assumed available through the star tracker. These initial conditions are used to propagate the quaternion $\mathbf{q}_{\text {prog }}$. The performance index $J 2$ is:

$$
J 2=\int_{t_{0}}^{t_{f}}\left\|\mathbf{q}_{\text {prog }}-\mathbf{q}_{\text {tru }}\right\| d t
$$

Subject to the dynamics Eq. (2.5), kinematics Eq. (2.1), and unity constraint of quaternion.

(3) An accurate dynamic model of CASSIOPE is required to solve this optimization problem. Toward that goal, the optimization problem is extended to include the principal moments of inertia in the design variables; that is the design variables vector 
become $\left(\omega_{x}, \omega_{y}, \omega_{z}, \phi, \theta, \psi, I_{x}, I_{y}, I_{z}\right)$. The initial inertia tensor $[I]$ is given in Eq. 2.14). The full inertia matrix Eq. (2.14) is used to compute the initial values of the principal moments of inertia using the direction cosine matrix $[A]$ as $[I]=[A]\left[I_{\text {diag }}\right][A]^{T}$. The direction cosine matrix $[A]$ is a function of the three Euler angles $[A(\phi, \theta, \psi)] \in \mathbb{R}^{3 \times 3}$ and it is unitary matrix and defined as follows:

$$
[A(\phi, \theta, \psi)]=\left[\begin{array}{ccc}
C \theta C \psi & C \theta S \psi & -S \theta \\
S \phi C \theta C \psi-C \phi S \psi & S \phi S \theta S \psi+C \phi S \psi & S \phi C \theta \\
C \phi S \theta C \psi+S \phi S \psi & C \phi S \theta S \psi-S \phi C \psi & C \phi C \theta
\end{array}\right]
$$

where $S$. is the sine and $C$. cosine. $\left[I_{\text {diag }}\right]$ is a diagonal matrix and its elements are the principal inertia tensor parameters in $X, Y$ and $Z$ directions. They are the singular values of the matrix $[I]$. By using the singular value decomposition method [92] Eq. (5.5), where $[\mathbf{M}]_{x}$ is replaced by $[I]$, the outputs will be $\left[I_{\text {diag }}\right]=[\Lambda]$ and $[A(\phi, \theta, \psi)]=[U]$. The initial principal moments of inertia are $I_{x}=215.9719\left[\mathrm{~kg} \cdot \mathrm{m}^{2}\right]$, $I_{y}=193.5539\left[\mathrm{~kg} \cdot \mathrm{m}^{2}\right]$ and $I_{z}=185.5468\left[\mathrm{~kg} \cdot \mathrm{m}^{2}\right]$. The initial values of the angular velocities are initial gyro real measurements. Moreover, the initial values of the three Euler angles are computed as follows; $\theta=-\cos ^{-1}\left(A_{13}\right), \psi=\tan ^{-1}\left(\frac{A_{12}}{A_{11}}\right)$, and $\phi=\tan ^{-1}\left(\frac{A_{23}}{A_{33}}\right)$. Where $A_{i j}$ represents the component of the row $i$ and column $j$ of the matrix $[A(\phi, \theta, \psi)]$. This constrained nonlinear multi-variable optimization problem is solved using the Matlab fmincon function. 
(4) The output from the optimization process (initial angular velocity, and inertia parameters) are used to find the angular velocity history via propagation. The optimized mean values of the principal inertia are very close to the initial values. In fact, the means and standard deviations are found to be: $\mu\left(I_{x}\right)=215.9752\left[\mathrm{~kg} \cdot \mathrm{m}^{2}\right]$, $\mu\left(I_{y}\right)=193.5545\left[\mathrm{~kg} \cdot \mathrm{m}^{2}\right], \mu\left(I_{z}\right)=185.5477\left[\mathrm{~kg} \cdot \mathrm{m}^{2}\right], \sigma\left(I_{x}\right)=0.0058\left[\mathrm{~kg} \cdot \mathrm{m}^{2}\right], \sigma\left(I_{y}\right)=$ $0.0047\left[\mathrm{~kg} \cdot \mathrm{m}^{2}\right]$ and $\sigma\left(I_{z}\right)=0.0008\left[\mathrm{~kg} \cdot \mathrm{m}^{2}\right]$. In order to check the performance of the optimization process, the error between the true attitude and the propagated one using the optimized parameters should be small. Figure 5.19, upper subplot, represents the principal rational error angle $\Phi$ between the true attitude and the propagated one which indicates a good optimization performance process.

The gyroscope bias is estimated. It is here assumed to be the difference between the mean value of the propagated angular velocity and the measured angular velocity (this is computed as the mean value of a polynomial fit for the measurements.) An Extended Kaman Filter (EKF) used to suppress the noises from these unbiased angular velocity measurements. The EKF uses the following three diagonal covariance matrices: initial state covariance $P=\operatorname{diag}\left(\begin{array}{lll}1 & 1 & 1\end{array}\right) * 1 e^{-5}$, measurement noise error covariance $R=\operatorname{diag}\left(\begin{array}{lll}1 & 1 & 1\end{array}\right) * 1 e^{-2}$, and process noise error covariance $Q=\operatorname{diag}\left(\begin{array}{lll}1 & 1 & 1\end{array}\right) * 1 e^{-5}$, where $\operatorname{diag}(x)$ means a diagonal matrix whose diagonal elements are $x$. Euler Eq. (2.5) is used for propagating the spacecraft angular velocity in the EKF, while the unbiased angular velocity is used as the measurement input to the EKF. The Jacobean matrix 
that will be used for computing the state transition matrix $[\phi]$ is as follows:

$$
[F(x)]=[I]^{-1}\left(\left[I \overline{\boldsymbol{\omega}}_{b o}\right]_{x}-\left[\overline{\boldsymbol{\omega}}_{b o}\right]_{x}[I]\right)
$$

The linearized form of the measurements matrix is

$$
[H]=\left[\mathbf{1}_{3 x 3}\right]
$$

Figure 5.20 shows the unbiased measurement versus the estimated states using an extended Kalman filter.

(5) The proposed algorithm here uses $\epsilon=3$. Using fig. 5.1, the following steps are performed to verify the magnetic field estimation algorithm:

1. At step time $t 1$, it is assumed that the magnetic field measurements are available and used in the control law. The time $t 1$ is the start point for the proposed algorithm.

2. At step time $t 2$, the spacecraft angular velocity measurements are also available. The magnetic field measurement along with the dipole moment command at $t 1$ are used to compute the spacecraft magnetic applied torque. Including also the reaction wheel torque and the computed gravity gradient torque, the resultant 
torque is used for propagating the spacecraft angular velocity. The EKF will use both the spacecraft angular velocity measurement and the propagated angular velocity to compute the refined angular velocity. In order to compute the spacecraft angular velocity derivative $\dot{\boldsymbol{\omega}}$ numerically using the five-stencil method, at least five consequence measurements are required with the same applied torque. Therefore, an interpolation is used to compute theses velocities. Once the $\dot{\boldsymbol{\omega}}$ is computed numerically, the magnetic field will be computed as discussed in section 5.2. This computed magnetic field is considered as the ambient magnetic field around the spacecraft and will be used by the control law to compute the required dipole moment that will be applied between $t 2$ and $t 3$ in fig. 5.1 .

3. At step time $t 3$, the same process and computations of $t 2$ will be repeated again to compute the magnetic field pseudo measurement $\mathbf{B}_{\text {sdo }}$ to be used. The $\mathbf{B}_{\text {sdo }}$ at step time $t 2$ is used instead of real magnetic field measurement for angular velocity propagation, Once the guess of $\mathbf{B}_{\text {sdo }}$ is available, it can be used to compute the required dipole moment $\mathbf{M}$ using Eq. (5.14, where the control term $\mathbf{T}_{r e q}$ is given in the telemetry data, to be applied between $t 3$ and $t 3+\delta T_{s}$, since the available time for activating the magnetic torque here is only $\delta T_{s}=0.07[\mathrm{~s}]$. Then after that, the magnetic rods will be switched off for desaturation and preparation for the magnetometer measurements. Till this point, the maximum allowable time for activating the magnetic rods is $0.27[\mathrm{~s}]$ for the proposed algorithm compared to $0.21[\mathrm{~s}]$ for the reference case. The 
magnetic field measurements are taken every $0.3[\mathrm{~s}]$ for the proposed algorithm compared to $0.1[\mathrm{~s}]$ for the reference case.

4. The process is repeated. The actions at step $t 4$ are exactly the same as those at step $t 1$.

Figure 5.21 shows a good match between the magnetic field measurements, labeled (True), and the magnetic field pseudo measurements, labeled (Estimated). Furthermore, the results demonstrate a good match between the given dipole moment, labeled (True), and the computed one, labeled (Computed) as shown in fig 5.22 . Figure 5.23 shows a good match between the magnetic torque, labeled (True), and the computed one, labeled (Computed). Figure 5.19 renders the angle $\lambda$ between the true and estimated torque and the angle $\alpha$ between the true and the magnetic field pseudo measurement vectors. For the second and third maneuvers, the magnetic field history for the true and the the magnetic field pseudo measurement values are shown in figs. 5.24 and 5.25 , which also demonstrate good performance.

For further assessment of estimating the magnetic field pseudo measurement and the two consequence vectors, the dipole moment and torque, six parameters are used.

Table 5.3 presents six parameters that assess the estimation quality in each of the three maneuvers considered above. For each maneuver, the first row presents these six parameters for the magnetic field pseudo measurement $\mathbf{B}_{\text {sdo }}$, the second row is for the 

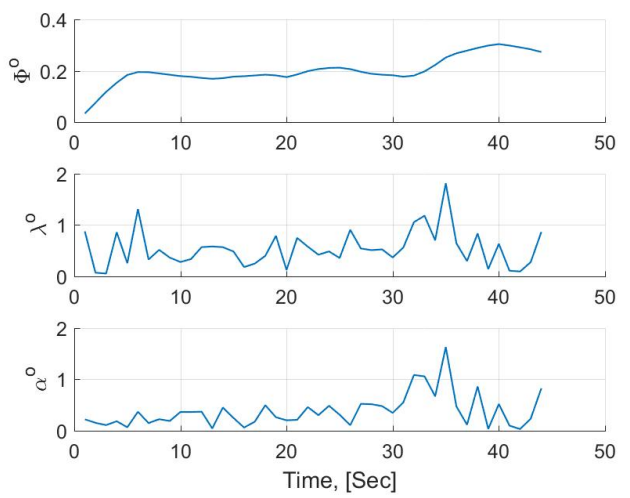

Figure 5.19: Attitude error in principal rotation angle $\Phi, \alpha$ and $\lambda$ histories for $1^{\text {st }}$ maneuver.
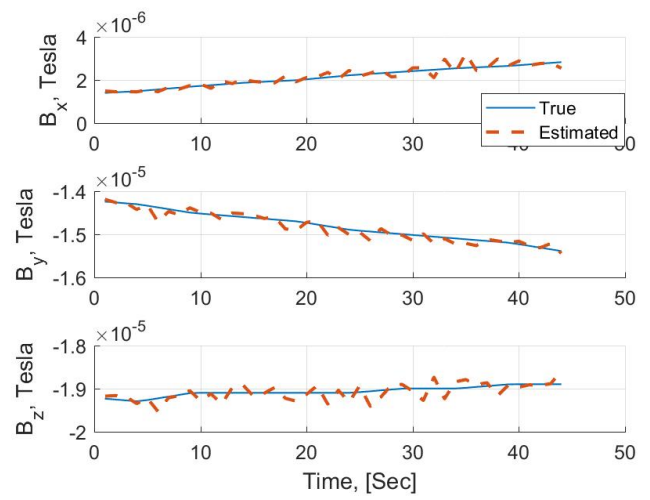

Figure 5.21: Magnetic field history for $1^{\text {st }}$ maneuver.

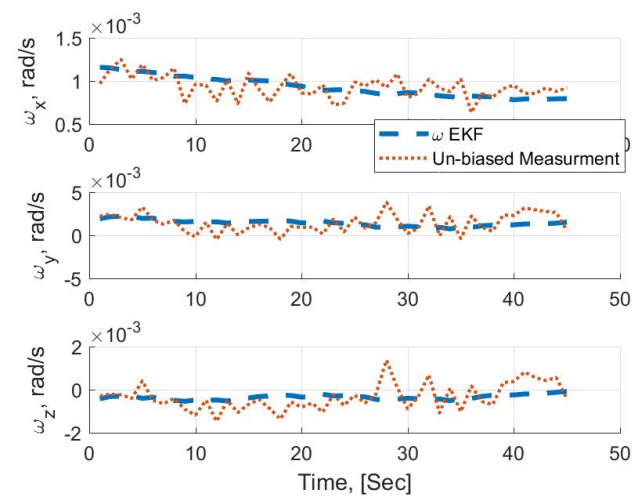

Figure 5.20: Angular velocities from unbiased gyroscope measurements and the EKF output for $1^{\text {st }}$ maneuver.
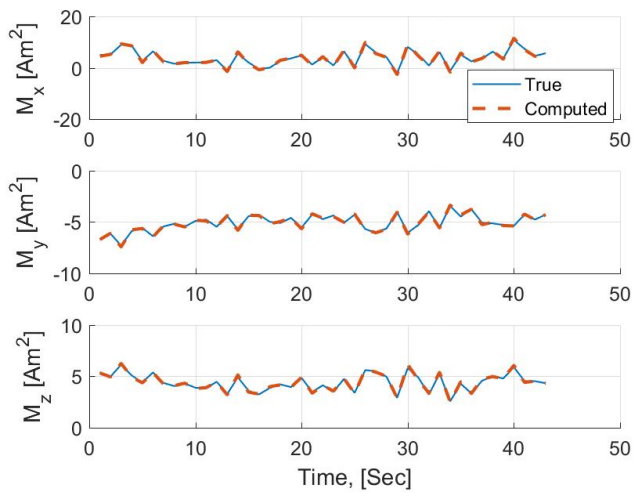

Figure 5.22: Dipole moment history for $1^{\text {st }}$ maneuver.

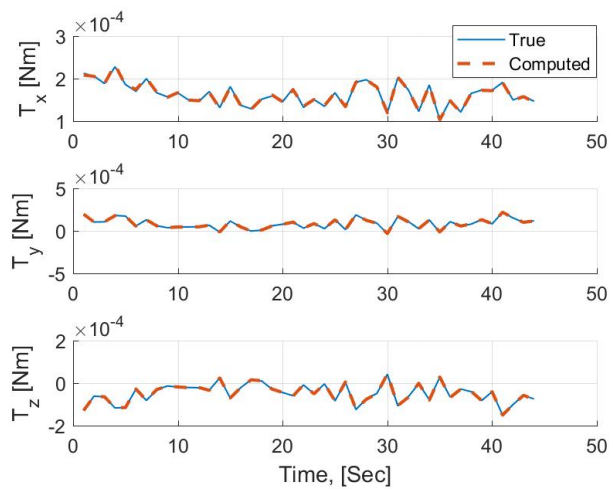

Figure 5.23: Torque history for $1^{\text {st }}$ maneuver 

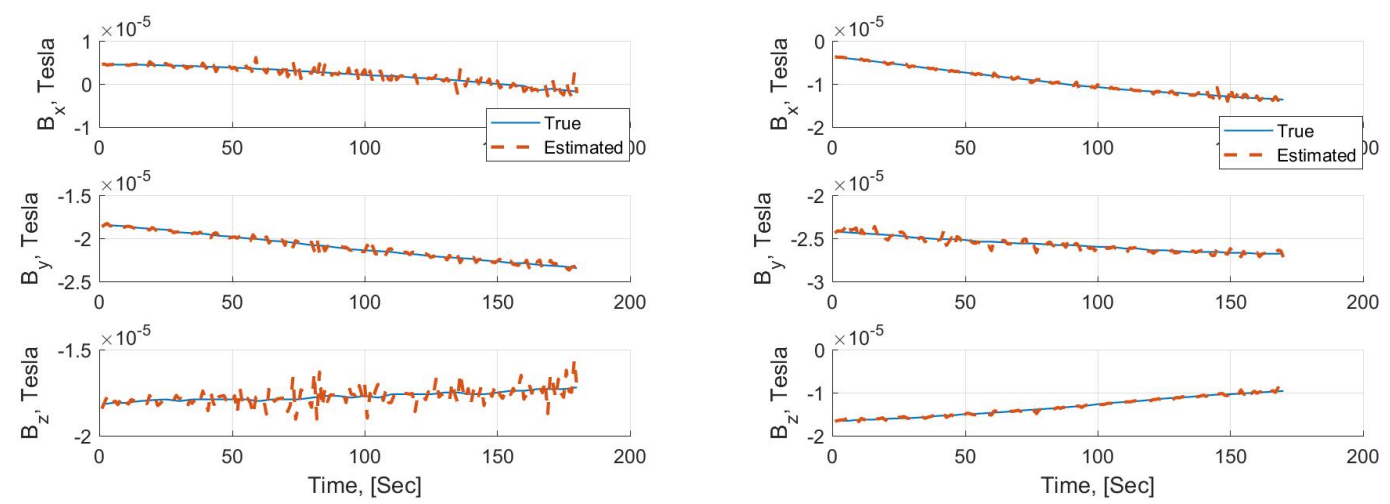

Figure 5.24: Magnetic field history for $2^{\text {nd }}$ maneuver.

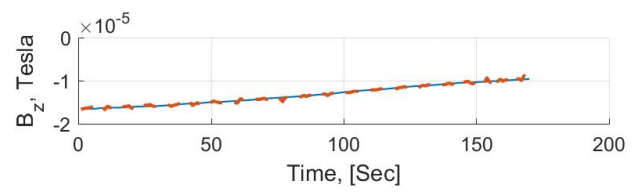

Figure 5.25: Dipole moment history for $3^{\text {rd }}$ maneuver.

Table 5.3

Validation parameters for three maneuvers

\begin{tabular}{cccccccc}
\hline No. & CC & SI & NRMSE & RMSE & MAE & Bias & \\
\hline \multirow{2}{*}{$1^{\text {st }}$} & 0.826 & 0.033 & 0.033 & $1.51 e^{-7}$ & $2.83 e^{-8}$ & $1.13 e^{-7}$ & $\mathrm{~B}$ \\
& 0.994 & 0.039 & 0.039 & 0.192 & 0.013 & 0.153 & $\mathrm{M}$ \\
& 0.999 & 0.013 & 0.013 & $1.40 e^{-6}$ & $3.56 e^{-8}$ & $1.07 e^{-6}$ & $\mathrm{~T}$ \\
\hline \multirow{2}{*}{$2^{\text {nd }}$} & 0.796 & 0.117 & 0.117 & $5.43 e^{-7}$ & $1.43 e^{-8}$ & $3.91 e^{-7}$ & $\mathrm{~B}$ \\
& 0.823 & 0.174 & 0.174 & 0.501 & 0.019 & 0.351 & $\mathrm{M}$ \\
& 0.997 & 0.053 & 0.053 & $2.96 e^{-6}$ & $1.126 e^{-7}$ & $2.14 e^{-6}$ & $\mathrm{~T}$ \\
\hline \multirow{2}{*}{$3^{\text {rd }}$} & 0.969 & 0.026 & 0.026 & $3.522 e^{-7}$ & $1.95 e^{-8}$ & $2.45 e^{-7}$ & $\mathrm{~B}$ \\
& 0.994 & 0.056 & 0.057 & 0.303 & 0.032 & 0.204 & $\mathrm{M}$ \\
& 0.996 & 0.034 & 0.033 & $4.35 e^{-6}$ & $1.49 e^{-7}$ & $2.86 e^{-6}$ & $\mathrm{~T}$ \\
\hline
\end{tabular}

dipole moment $\mathbf{M}$, and the third row is for the estimated torque $\mathbf{T}$. These parameters are the correlation coefficient $(\mathrm{CC})$, the scatter index (SI), the normalized root mean square error (NRMSE), mean absolute error (MAE), Bias, and root mean square error (RMSE). The values presented in Table 5.3 are the mean values of the six parameters. Good performance can be observed from this table in the three maneuvers. 


\subsection{Conclusion}

For magnetic attitude maneuvers, an algorithm for computing the ambient magnetic field based on the spacecraft angular velocity measurements is developed. This algorithm was used in this study to enable longer operation of the magnetic rods, and hence reducing the maneuver time, power consumption and reducing the attitude steady state error as proofed analytically by considering the duty cycle effect during the stability analysis. The results confirmed the claimed advantages. A core algorithm in this work is the algorithm that computes the ambient magnetic field pseudo measurement based on the angular velocity rate. Real data from the CASSIOPE spacecraft were used to validate this core algorithm. The validation demonstrated that the magnetic field can be computed with a good accuracy. The computational cost of the proposed algorithm is significantly higher than the reference algorithm due to the need for in-line optimization and eigenvalues and eigenvectors computation. 



\section{Chapter 6}

\section{A Spacecraft Attitude Estimation}

\section{Algorithm for Efficient Magnetic}

\section{Attitude Maneuvers}

In magnetic attitude maneuvers, magnetic rods and magnetometers usually operate alternatively, to avoid the rods' noise effect on magnetometers. Because of that, the magnetic rods operation time is reduced and there will be no control authority over the spacecraft during the magnetometer measurement period. In the previous chapter, a control scheme is developed that enable longer magnetic rods operation. Which in turn will perform maneuvers in less time, using less power for the magnetic rods, 
and reduce the attitude steady state error. The key concept is replacing the real magnetic field measurement to what we called magnetic field pseudo measurement. By applying a known command on the spacecraft and measuring the spacecraft response (rotation), it is possible to compute the ambient magnetic field around the spacecraft. The system mathematical singularity is solved using the Tikhonov regularization approach. However, this method is suffering from the high computational load demands due to in-line optimization, and eigenvalues and eigenvectors calculations.

However, this chapter presents an estimation approach, for estimating both the spacecraft attitude and the magnetic field. An extended Kalman filter (EKF) is used in the proposed approach. A relatively simple and fast dynamic model is developed for use in the EKF. This chapter is different from the developed control scheme in the previous chapter in the following aspects: 1) computing the pseudo measurements of the magnetic field by measuring the spacecraft response to a known control command is implemented here from a geometric point of view, as opposed to the Tikhonov regularization technique used in [62], 2) this work uses an attitude sensor, in addition to the magnetometers, in the Multiplicative Extended Kalman Filter (MEKF), and 3) the computational load of the proposed work here is much less than that in chapter 5 [62], and is comparable to the computational load of most exciting magnetic control techniques in the literature, as will be shown in the simulation results. Therefore, the control scheme developed in this chapter will be referred to as (attitude dependant scheme). Whereas the one that developed in chapter 5 will be referred to as (attitude 
independent scheme).

\subsection{Attitude Determination and Control System}

This section presents the proposed algorithm for estimating the attitude and magnetic field for spacecraft attitude regulation. Here the target will be earth pointing maneuver. First, a reference Attitude Determination and Control System (ADCS) algorithm is discussed highlighting the challenges that will be addressed in the proposed algorithm. This reference algorithm will be used in the simulation section for comparison and performance assessment of the proposed ADCS.

In the reference ADCS algorithm, the magnetic rods and the magnetometers are assumed to operate at alternate times, as shown in fig. 6.1. Detailed discussion on the time structure of the magnetic rods and magnetometer operation cycle is provided in section 1.2.2. The lower part of fig. 6.1 shows several cycles of the magnetic rods and the magnetometer activation periods. Also, the attitude estimation algorithm typically updates the estimates for the quaternion $\tilde{\mathbf{q}}$, the spacecraft angular velocity $\tilde{\boldsymbol{\omega}}$, and gyroscope bias $\boldsymbol{\beta}$ each cycle. The upper part of fig. 6.1 shows the estimated and measured quantities, and the times at which they are collected. The measurements are: the angular velocity $\boldsymbol{\omega}$, the magnetic field $\mathbf{B}$, and the sun direction $\mathbf{V}_{\text {sun }}$ for sun sensor (any attitude sensor can be used such as earth horizon sensor). 


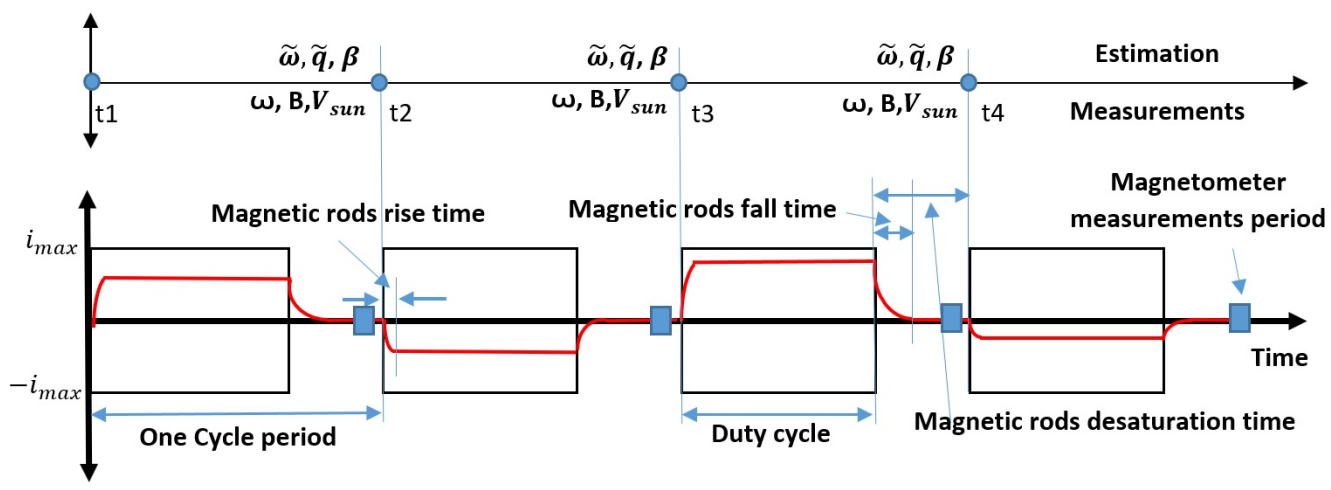

Figure 6.1: In the reference ADCS algorithm, the magnetic rods operate on a duty cycle, alternating with magnetometers to avoid high noise on the latter.

In this study, the proposed ADCS algorithm increases the duty cycle. This is achieved by estimating the magnetic field parameters at some of the cycles, and hence eliminating the need for magnetic field measurements in these cycles. At the core of the proposed ADCS is an algorithm that estimates the magnetic field in these cycles (at times $t_{2}$ and $t_{3}$ ) when the magnetometer measurements are not available; this algorithm is presented in section 6.1.1. The upper part of fig. 6.2 illustrates the measured and estimated quantities in each cycle. At each of the times $t_{2}$ and $t_{3}$, a calculated magnetic field $\mathbf{B}_{s d o}$ is used as a pseudo measurement input to the estimator, instead of the input magnetic field measurement $\mathbf{B}$, which is available only at times $t_{1}$ and $t_{4}$. The extended Kalman filter used in the proposed ADCS is presented in section 6.1.2.

Same control law described in section 5.3 is adopted for Earth pointing maneuver as follows:

$$
\mathbf{T}_{r e q}=-\left(\zeta^{2} k_{p} \mathbf{q}_{v}+\zeta k_{d} \boldsymbol{\omega}_{b o}\right)
$$



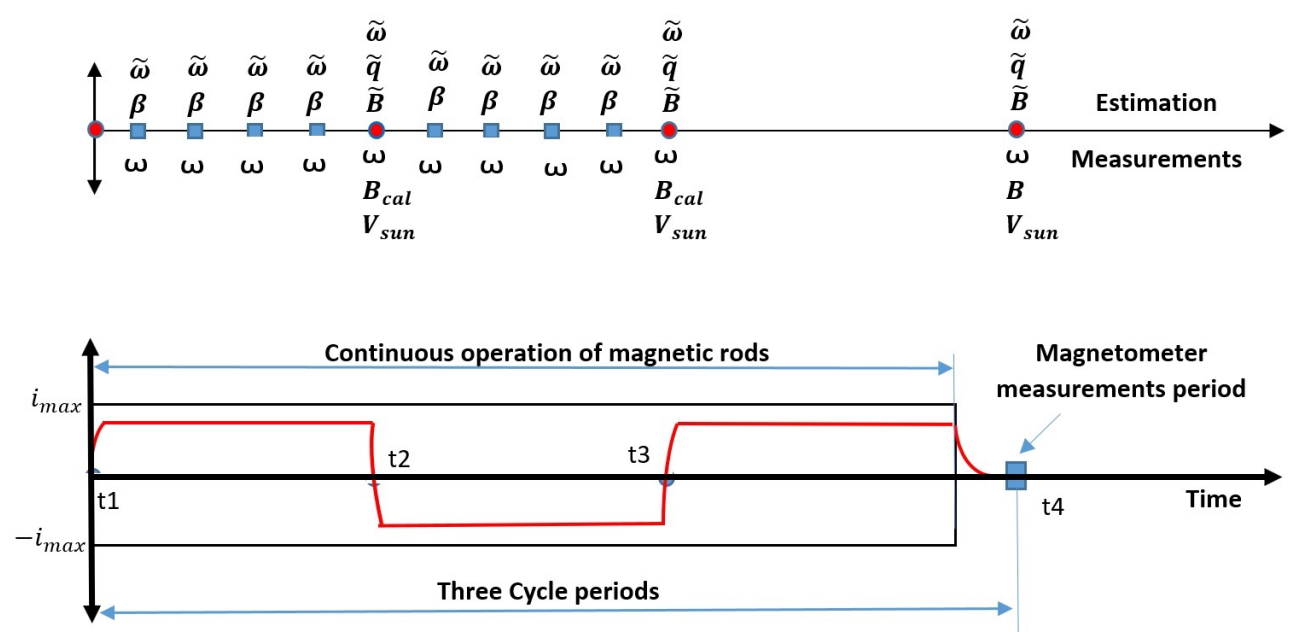

Figure 6.2: The proposed magnetic rods operation.

Where The control objective here is to derive the spacecraft body frame $b$ to align with the orbit frame $o$. Therefore, from Eq. 2.2$)$, it follows that $[R(\mathbf{q})]=\mathbf{1}_{3 x 3}$. Thus the objective is to design control law so that $\boldsymbol{\omega}_{b o} \rightarrow 0$ and $\mathbf{q}_{v} \rightarrow 0$.

\subsubsection{Magnetic Field Pseudo Measurement}

The following discussion is dedicated to find the best guess of the magnetic field pseudo Measurement vector $\mathbf{B}_{s d o}$. When the magnetometer measurements are not available, for projection based control strategy such as the one that used in this entire study. However, here, the mathematical singularity problem will be solved from a geometric point of view to avoid the high computational demands that required by the Tikhonov regularization approach described in section 5.2. The process starts by computing the spacecraft applied torque for known dipole command by measuring 
the spacecraft response (rotation) as we did in section 5.2.1 using dynamic model of the spacecraft Eq. (2.5).

It is noted that the assumption of having a frequency of gyroscope measurements collection at least five times higher than the control command frequency, is a reasonable assumption, as discussed in [105]. The gyroscope measurements are subject to gyroscope drift rate bias, random walk and misalignment errors. Therefore, an $\mathrm{EKF}_{\omega}$ is used for estimating the spacecraft angular velocity and bias vector whenever the gyroscope measurements are available. This $\mathrm{EKF}_{\omega}$ outputs the estimated angular velocity, which is used for computing $\dot{\boldsymbol{\omega}}$ as seen in the upper part of fig. 6.2, between times $t 1-t 2$ and $t 2-t 3$. The propagation step in the $\mathrm{EKF}_{\omega}$ inside the period $t_{i}-t_{i+1}$ is carried out assuming a constant magnetic field, which is obtained at $t_{i}$, see also fig. 6.2. The Angular velocity is filtered when estimating the bias vector in the $\mathrm{EKF}_{\omega}$. The system model equations are Eqs. 2.5), 6.2 and 6.3):

$$
\begin{gathered}
\boldsymbol{\omega}_{m e s}=\boldsymbol{\omega}+\boldsymbol{\beta}+\boldsymbol{\eta}_{v} \\
\dot{\boldsymbol{\beta}}=\boldsymbol{\eta}_{u}
\end{gathered}
$$

where $\boldsymbol{\omega}_{\text {mes }} \in \mathbb{R}^{3}$ is the gyroscope output, $\boldsymbol{\beta} \in \mathbb{R}^{3}$ is the gyroscope bias vector, $\boldsymbol{\eta}_{v} \in \mathbb{R}^{3}$ is the random drift noise and $\boldsymbol{\eta}_{u} \in \mathbb{R}^{3}$ is the random walk drift noise. The sate vector is $\mathbf{x}=\left[\begin{array}{ll}\boldsymbol{\omega}^{T} & \boldsymbol{\beta}^{T}\end{array}\right]^{T}$. The Jacobean matrix that will be used for computing 
the state transition matrix $[\phi]$ is as follows:

$$
[F(x)]=\left[\begin{array}{cc}
{[I]^{-1}\left(\left[I \overline{\boldsymbol{\omega}}_{b o}\right]_{x}-\left[\overline{\boldsymbol{\omega}}_{b o}\right]_{x}[I]\right)} & -\left[\mathbf{1}_{3 x 3}\right] \\
{\left[\mathbf{0}_{3 x 3}\right]} & {\left[\mathbf{0}_{3 x 3}\right]}
\end{array}\right]
$$

The state transition matrix $[\phi]$ is approximated by $[\phi] \approx\left[\mathbf{1}_{3 X 3}\right]+[F(x)] d t$ for small time step $d t$ [106]. The linearized form of the measurements matrix is

$$
[H]=\left[\begin{array}{ll}
{\left[\mathbf{1}_{3 x 3}\right]} & {\left[\mathbf{0}_{3 x 3}\right.}
\end{array}\right]
$$

The derivative of the angular velocity $\dot{\omega}$ can be computed using Eq (5.2). Then, an estimation of the torque $\tilde{\mathbf{T}}$ can be computed using the Euler dynamic model Eq (2.5). Equations (5.14) and (2.7) imply that the torque, dipole moment and magnetic field vectors are orthogonal to each other in the ideal case when $\mathbf{B}_{s d o}=\mathbf{B}$. Assuming that $\mathbf{B}_{\text {sdo }}$ remains close to $\mathbf{B}$, and given an estimate for dipole moment $\tilde{\mathbf{M}}$ and $\tilde{\mathbf{T}}$, it is possible to compute the pseudo measurement vector, $\mathbf{B}_{s d o}$, from a geometric point of view as follows. The unit vector of the $\mathbf{B}_{\text {sdo }}$ vector can be computed as follows :

$$
\hat{\mathbf{B}}_{s d o}=\frac{\tilde{\mathbf{T}}}{\|\tilde{\mathbf{T}}\|} \times \frac{\tilde{\mathbf{M}}}{\|\tilde{\mathbf{M}}\|}=\hat{\tilde{\mathbf{T}}} \times \hat{\tilde{\mathbf{M}}}
$$

In addition, the magnitude of the pseudo measurement of ambient magnetic field 
vector can be computed as follows:

$$
\left\|\mathbf{B}_{\text {sdo }}\right\|=\frac{\|\tilde{\mathbf{T}}\|}{\|\tilde{\mathbf{M}}\|}
$$

Therefore, the pseudo measurements of ambient magnetic field vector will be

$$
\mathbf{B}_{s d o}=\frac{\|\tilde{\mathbf{T}}\|}{\|\tilde{\mathbf{M}}\|} \hat{\tilde{\mathbf{T}}} \times \hat{\tilde{\mathbf{M}}}
$$

Further analysis over the computation of $\mathbf{B}_{s d o}$ is carried out to check the effect of the error in the torque and the dipole moment vectors, $\delta \mathbf{T}$ and $\delta \mathbf{M}$, respectively. Where $\delta \mathbf{B}, \delta \mathbf{T}$ and $\delta \mathbf{M}$ represent error vectors added to the vectors $\mathbf{B}, \mathbf{T}$ and $\mathbf{M}$ to get the estimated vectors $\mathbf{B}_{s d o}, \tilde{\mathbf{T}}$, and $\tilde{\mathbf{M}}$ as $\mathbf{B}_{s d o}=\mathbf{B}+\delta \mathbf{B}, \tilde{\mathbf{T}}=\mathbf{T}+\delta \mathbf{T}$ and $\tilde{\mathbf{M}}=\mathbf{M}+\delta \mathbf{M}$.

The torque error is due to the noises in the gyro measurements and the errors in modeling the external disturbance torques such as gravity gradient, residual dipole moment, and aerodynamic. In addition to the sensitivity of the spacecraft dynamic model to the uncertainty in the moment of inertia. The error in the dipole moment vector $\delta \mathbf{M}$ is due to the uncertainty in the actuator's model, current noises and digitization process in the measured commanded current to the actuators. The estimate of the torque applied on the spacecraft is expressed as:

$$
\tilde{\mathbf{T}}=\tilde{\mathbf{M}} \times \mathbf{B}_{s d o}
$$


Therefore, the torque error vector is as follows:

$$
\delta \mathbf{T}=\tilde{\mathbf{M}} \times \delta \mathbf{B}+\delta \mathbf{M} \times \mathbf{B}
$$

The most dominant term in Eq. 6.10) in the right hand side is the first term. Therefore, Eq. 6.10 can be approximated as follows:

$$
\delta \mathbf{T} \approx \tilde{\mathbf{M}} \times \delta \mathbf{B}
$$

Where a bound $v \in \mathbb{R}^{3}$ on the ambient magnetic field error vector, $|\delta \mathbf{B}| \leq v$, is added. To make this analysis easier to visualize and as we concern with direction of magnetic field for the projection based control scheme, we express the error in each vector in terms of the corresponding angle. Consider fig 1.1, it is possible to express the error vector in the ambient magnetic field, $\delta \mathbf{B}$ in terms of the angle $\alpha$ as follows:

$$
|\alpha|<\alpha_{v}
$$

where the angle $\alpha$ between the best guess $\mathbf{B}_{s d o}$ vector and the true one $\mathbf{B}$ should be kept under a threshold angle $\alpha_{v}$. The angle $\lambda$ is the angle between the true applied torque and the estimated torque $\tilde{\mathbf{T}}$, and the angle $\eta$ is the angle between the true dipole moment and the estimated torque $\tilde{\mathbf{M}}$.

Figure 6.3 shows the typical relation between the angles $\alpha$ and $\eta$ for different values 


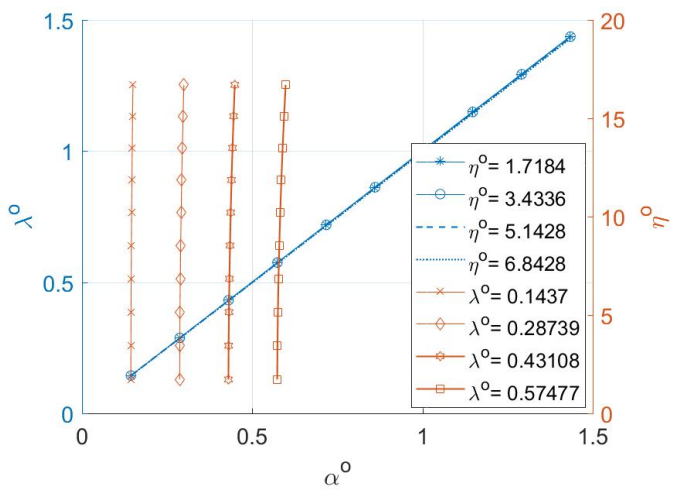

Figure 6.3: The relation between $\alpha$ and each of $\lambda$ (the left vertical axis) and $\eta$ (the right vertical axis). The angles $\lambda$ and $\eta$ are varied and the corresponding change in $\alpha$ is computed.

of $\lambda$ where $\eta$ is presented on the right vertical axis. For a wide range of the angle $\eta$, there is almost no change in the angle $\alpha$. In the same figure, the relation between the angles $\alpha$ and $\lambda$ for different values of $\eta$ is plotted, where $\lambda$ is presented on the left axis. The correlation between $\alpha$ and $\lambda$ is strong; consequentially $\lambda$ has a significant impact on $\delta \mathbf{B}$. Figure 5.19 shows the angles $\lambda$ and $\alpha$ history for the real data case presented in section 5.4. In that real case, there is an agreement between the computed torque and the true one to an acceptable accuracy as demonstrated in Fig. 5.19. Hence, it can be concluded that the error in $\alpha$ is small, see the third subplot of Fig. 5.19, in the estimation process described above, and hence the error $\delta \mathbf{B}$ is small. This completes the process of computing the vector of the ambient magnetic field $\mathbf{B}_{s d o}$, and completes the calculations needed in the proposed control algorithm. 


\subsubsection{Attitude estimation}

A continuous-discrete MEKF [104] is used to estimate the following: the attitude

quaternion $\tilde{\mathbf{q}}$, the angular velocity $\tilde{\boldsymbol{\omega}}$, and the magnetic field $\tilde{\mathbf{B}}$. The measurements used by this MEKF are the angular velocity from the gyroscope, the direction of the sun from the sun sensor (or any inertial sensor), the magnetic field real measurements $\mathbf{B}$ at times $t_{1}$ and $t_{4}$, and magnetic field pseudo measurements $\mathbf{B}_{\text {sdo }}$ at times $t_{2}$ and $t_{3}$, see fig. 6.2. The state vector is $\mathbf{x}_{k}=\left[\boldsymbol{\omega}_{b o}^{T} \mathbf{q}^{T} \mathbf{B}^{T}\right]^{T} \in \mathbb{R}^{10}$, and the error state vector is $\delta \mathbf{x}_{k}=\left[\delta \boldsymbol{\omega}_{b o}^{T} \delta \mathbf{q}_{v}^{T} \delta \mathbf{B}^{T}\right]^{T} \in \mathbb{R}^{9}$, where $\delta \mathbf{q}_{v} \in \mathbb{R}^{3}$ is the quaternion vector part error. Let $\mathbf{x}_{k \mid k}$ be the posterior estimate, and $\mathbf{x}_{k \mid k-1}$ be the a prior estimate. The propagation and update steps of the MEKF are presented below.

\section{State propagation}

During the propagation step, the full state is integrated numerically, using a $4^{\text {th }}$ order Runge-Kuta integration for the nonlinear kinetic equations in Eq (2.5), and for the kinematic model in $\mathrm{Eq}$ (2.1). However, for the propagation of the magnetic field vector $\mathbf{B}$, it is assumed that the derivative of the $\mathbf{B}$ vector at the orbit frame does not change (i.e. ${ }^{o} \dot{\mathbf{B}}=0$ ) during the propagation step. This assumption is acceptable for a small time step [51]. Consider the posterior estimated earth magnetic field vector 
$\mathbf{B}_{k-1 \mid k-1}$, computed at the time step $k-1$. The quaternion conjugate of the posterior quaternion update at the time step $k-1$ is $\mathbf{q}_{k-1 \mid k-1}^{-1}$. Then we can write:

$$
{ }^{o} \mathbf{B}_{k-1 \mid k-1}=\left[R\left(\mathbf{q}_{k-1 \mid k-1}^{-1}\right)\right] \mathbf{B}_{k-1 \mid k-1}
$$

where $\left[R\left(\mathbf{q}_{k-1 \mid k-1}^{-1}\right)\right]$ is the transformation matrix from the body frame to the orbit frame. The ${ }^{o} \mathbf{B}_{k-1 \mid k-1}$ can be transformed to the body frame using the transformation matrix $\left[R\left(\mathbf{q}_{k \mid k-1}\right)\right]$; hence assuming that ${ }^{o} \dot{\mathbf{B}}_{k}=0$, we can write:

$$
\mathbf{B}_{k \mid k-1}=\left[R\left(\mathbf{q}_{k \mid k-1}\right)\right]\left[R\left(\mathbf{q}_{k-1 \mid k-1}^{-1}\right)\right] \mathbf{B}_{k-1 \mid k-1}
$$

Equation 6.14 can be used to propagate the magnetic field vector. The covariance matrix $[P]$ propagates in time according to the Riccati equation. However, for a simpler numerical implementation, the following approximation is utilized [107]:

$$
[P]_{k \mid k-1}=\left[\phi_{t_{k}, t_{k-1}}\right]\left[P_{k-1 \mid k-1}\right]\left[\phi_{t_{k}, t_{k-1}}\right]^{T}+\left[Q_{k-1}\right]
$$

where $\left[Q_{k-1}\right]$ is the discrete-time process covariance matrix, and $\left[\phi_{t_{k}, t_{k-1}}\right]$ is the state transition matrix. In order to find the state transition matrix $\left[\phi_{t_{k}, t_{k-1}}\right]$, a linearization for the nonlinear models is carried out as detailed below. 
The derivative of the ambient magnetic field vector can be computed using the transport theorem as follows [4, 82]:

$$
\dot{\mathbf{B}}=[R(\mathbf{q})]^{o} \dot{\mathbf{B}}+[\mathbf{B}]_{x} \boldsymbol{\omega}_{b o}
$$

Equation. (2.4) is substituted into Eq. (6.16) assuming that ${ }^{\circ} \dot{\mathbf{B}}=0$ (small time step.) The transformation matrix $[R(\mathbf{q})]$ in Eq. (6.16) is approximated for small angles as: $[R(\mathbf{q})] \approx[\mathbf{1}]-2\left[\mathbf{q}_{v}\right]_{x}$. The linearized first order Taylor expansion version of Eq. 6.16) results in the small-signal dynamic equation of the magnetic field as follows:

$$
\delta \dot{\mathbf{B}}(t)=[\overline{\mathbf{B}}]_{x} \delta \boldsymbol{\omega}_{b o}-2[\overline{\mathbf{B}}]_{x}\left[\overline{\boldsymbol{\omega}}_{o i}\right]_{x} \delta \mathbf{q}_{v}-\left(\left[\overline{\boldsymbol{\omega}}_{b i}\right]_{x}-\left[\overline{\boldsymbol{\omega}}_{o i}\right]_{x}\right) \delta \mathbf{B}
$$

Similarly, Eq. (2.5) and Eq. (2.7) can be used to write the small-signal dynamic equation of the spacecraft angular velocity, where the disturbance torques are dropped (disturbance torques are assumed to have expected values of zero.) The small-signal dynamic equation of the angular velocity becomes:

$$
\delta \dot{\boldsymbol{\omega}}_{b o}(t)=[I]^{-1}\left(\left(\left[I \overline{\boldsymbol{\omega}}_{b o}\right]_{x}-\left[\overline{\boldsymbol{\omega}}_{b o}\right]_{x} I\right) \delta \boldsymbol{\omega}_{b o}+[\overline{\mathbf{M}}]_{x} \delta \mathbf{B}-[\overline{\mathbf{B}}]_{x} \delta \mathbf{M}\right)
$$


Similarly, the small-signal dynamic equation of the quaternion is:

$$
\delta \dot{\mathbf{q}}_{v}(t)=0.5\left[\mathbf{1}_{3 x 3}\right] \delta \boldsymbol{\omega}_{b o}-\left[\overline{\boldsymbol{\omega}}_{b o}\right]_{x} \delta \mathbf{q}_{v}
$$

The state transition matrix $[\phi]$ can be be approximated as $[\phi] \approx\left[\mathbf{1}_{9 x 9}\right]+[F(x)] d t$ for small time step $d t[106]$, where $[F(x)]$ is the Jacobian matrix. The latter can be computed from Eqs. 6.17), 6.18) and 6.19) to get:

$$
[F(x)]=\left[\begin{array}{ccc}
{[I]^{-1}\left(\left[I \overline{\boldsymbol{\omega}}_{b o}\right]_{x}-\left[\overline{\boldsymbol{\omega}}_{b o}\right]_{x}[I]\right)} & {\left[\mathbf{0}_{3 x 3}\right]} & {[I]^{-1}[\overline{\mathbf{M}}]_{x}} \\
0.5\left[\mathbf{1}_{3 x 3}\right] & -\left[\overline{\boldsymbol{\omega}}_{b o}\right]_{x} & {\left[\mathbf{0}_{3 x 3}\right]} \\
{[\overline{\mathbf{B}}]_{x}} & -2[\overline{\mathbf{B}}]_{x}\left[\overline{\boldsymbol{\omega}}_{o i}\right]_{x} & {\left[\overline{\boldsymbol{\omega}}_{o i}\right]_{x}-\left[\overline{\boldsymbol{\omega}}_{b i}\right]_{x}}
\end{array}\right]
$$

where $(\cdot)$ are the nominal values and they are the apriori propagated values of the state vector.

\section{State update}

A linearizion of the measurement model about the apriori state estimate is here carried out for use in the EKF. The sun sensor measurement is $\mathbf{V}_{\text {sun }}$, where:

$$
\mathbf{V}_{\operatorname{sun}(k)}=\left[R\left(\mathbf{q}_{k \mid k-1}\right)\right]^{o} \mathbf{V}_{\operatorname{sun}(k)}
$$


Assuming small angles, the transformation matrix can be approximated as: $[R(\mathbf{q})]=$ $[R(\overline{\mathbf{q}})][R(\delta \mathbf{q})] \approx\left[R\left(\mathbf{q}_{k \mid k-1}\right)\right]\left(\left[\mathbf{1}_{3 x 3}\right]+\left[\delta \mathbf{q}_{v}\right]_{x}\right)$. Using this approximation, the error in the sun direction can be approximated as follows:

$$
\mathbf{V}_{\operatorname{sun}(k \mid k-1)}-\overline{\mathbf{V}}_{\operatorname{sun}(k \mid k-1)} \approx 2\left[\overline{\mathbf{V}}_{\operatorname{sun}(k \mid k-1)}\right]_{x} \delta \mathbf{q}_{v}
$$

The linearized small error measurement model about the apriori state estimate can be written as follows:

$$
\mathbf{Z}_{k}=\left[H_{k}\right] \delta \mathbf{x}=\left[\begin{array}{ccc}
{\left[\mathbf{1}_{3 x 3}\right]} & {\left[\mathbf{0}_{3 x 3}\right]} & {\left[\mathbf{0}_{3 x 3}\right]} \\
{\left[\mathbf{0}_{3 x 3}\right]} & 2\left[\overline{\mathbf{V}}_{\operatorname{sun}(k \mid k-1)}\right]_{x} & {\left[\mathbf{0}_{3 x 3}\right]} \\
{\left[\mathbf{0}_{3 x 3}\right]} & {\left[\mathbf{0}_{3 x 3}\right]} & {\left[\mathbf{1}_{3 x 3}\right]}
\end{array}\right] \delta \mathbf{x}
$$

where $\mathbf{Z}_{k}$ is the small-signal (error) measurement vector. At each measurement time a Kalman gain is computed using Eq. (6.24). The measurements include the gyroscope, the sun sensor measurements, and the magnetic field $\left(\mathbf{B}\right.$ or $\left.\mathbf{B}_{s d o}\right)$.

$$
\left[K_{k}\right]=\left[P_{k \mid k-1}\right]\left[H_{k}\right]^{T}\left(\left[H_{k}\right]\left[P_{k \mid k-1}\right]\left[H_{k}\right]^{T}+\left[R_{k}\right]\right)^{-1}
$$

The update step is carried out for each of the $\mathbf{q}, \boldsymbol{\omega}$, and $\mathbf{B}$ states differently. The 
quaternion is updated using a quaternion multiplication as shown in Eq. 6.25).

$$
\mathbf{q}_{k \mid k}=\left[\begin{array}{c}
\sqrt{1-\left\|\delta \mathbf{q}_{v}\right\|^{2}} \\
\delta \mathbf{q}_{v}
\end{array}\right] \otimes \mathbf{q}_{k \mid k-1}
$$

where $\otimes$ represents quaternion product. The magnetic field $\mathbf{B}$, on the other hand, is updated on two steps. Recall that the propagation step of $\mathbf{B}$ in Eq. 6.14 used $\mathbf{q}_{k \mid k-1}$. Now that $\mathbf{q}_{k \mid k}$ is available, the latter is used to get a better propagation of B. This is carried out as follows: $\mathbf{B}_{k \mid k-1}^{+}=\left[R\left(\mathbf{q}_{k \mid k}\right)\right] \mathbf{B}_{k \mid k-1}$. Then the magnetic field is updated using this new propagated vector $\mathbf{B}_{k \mid k-1}^{+}$along with the error in magnetic field vector $\delta \mathbf{B}_{k \mid k}$, which is computed using the associated part of the Kalman gain and the measurements (or pseudo measurements) as follows:

$$
\begin{aligned}
\mathbf{B}_{k \mid k} & =\mathbf{B}_{k \mid k-1}^{+}+\delta \mathbf{B}_{k \mid k} \\
\boldsymbol{\omega}_{k \mid k} & =\boldsymbol{\omega}_{k \mid k-1}+\delta \boldsymbol{\omega}_{k \mid k}
\end{aligned}
$$

Where the spacecraft angular velocity is updated using the standard conventional approach Eq. 6.27). The estimated angular velocity bias vector (between $t_{i}$ times) from $\mathrm{EKF}_{\omega}$ is used here to update the angular velocity and will not be estimated at times $t_{i}$ to reduce the computational cost, see the upper plot fig. 6.2. The estimation 
error covariance matrix $[P]$ is updated as follows:

$$
\left[P_{k \mid k}\right]=\left(\left[\mathbf{1}_{9 x 9}\right]-\left[K_{k}\right]\left[H_{k}\right]\right)\left[P_{k \mid k-1}\right]
$$

\subsection{Numerical simulations}

The goal of this section is to compare the proposed ADCS algorithm, which is shown in fig. 6.2, to a reference standard algorithm. Then we will provide comparison between the magnetic field pseudo measurement methods later. The spacecraft hardware configuration, orbital, control algorithms parameters are the same as presented in section 5.4

\section{Case study}

For this sample case, fig. 6.4 depicts the time behavior of the satellite's quaternion for the reference case, (labeled "Ref"), along with the time behavior of the satellite's quaternion for the proposed algorithm with $\epsilon=5$, (labeled "Proposed $\epsilon=5$ "). Figure 6.4 has three columns of subplots; the left column shows the time history in the first orbit, the middle column shows the time history in the following three orbits, and the right column shows the time history from the fifth orbit until the end of the ten-orbits 


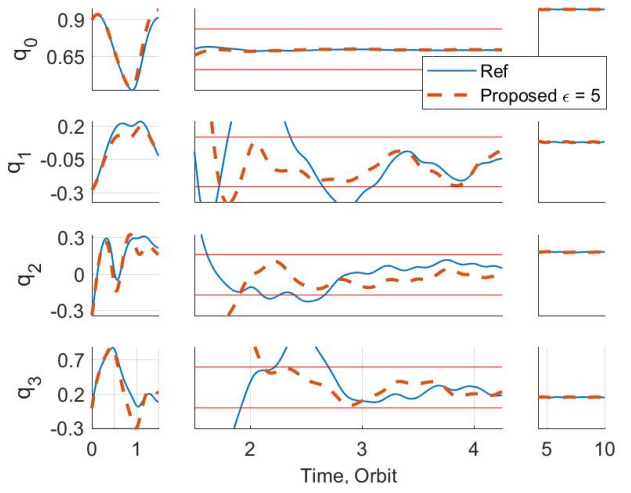

Figure 6.4: Spacecraft quaternion history over time.

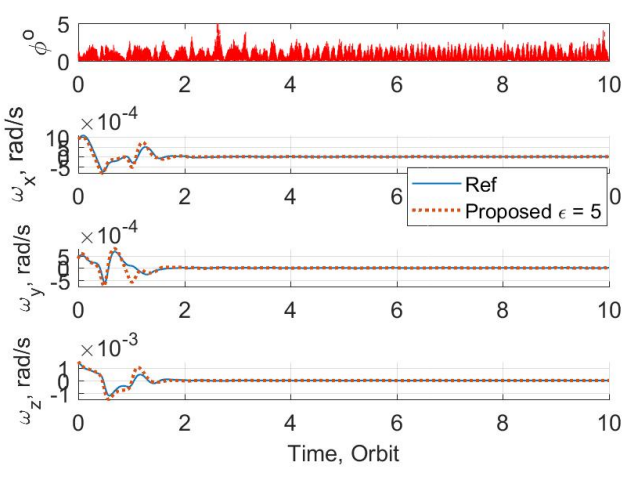

Figure 6.5: Spacecraft angular velocity and principal rotation angle error.

simulation period. The two horizontal lines in the middle column are the limits of the desired attitude. As shown in fig. 6.4, the proposed algorithm can settle at the desired attitude faster than the reference algorithm. The error between the true attitude and the desired one in terms of the principal rotation angle $\phi$ is kept less than $2^{\circ}$ most of the time as shown in the upper subplot of fig. 6.5. Figure 6.5 depicts the time behavior of the satellite's angular velocity of the body frame w.r.t the orbit frame. The proposed algorithm is able to preserve almost zero angular velocity vector after settling the spacecraft at the desired attitude, as shown in the lower three subplots in fig 6.5 ,

More on the comparison between the two algorithms is discussed next. Figure 6.6 represents the magnetic field time history of the estimated and true values. As can be seen, a good match has been achieved. Later, six parameters will be presented to assess the ambient magnetic field estimation performance. 


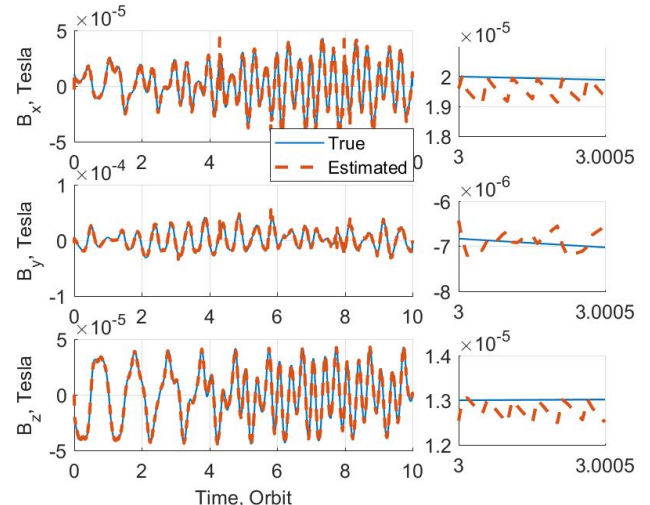

Figure 6.6: Ambient magnetic field history over time.

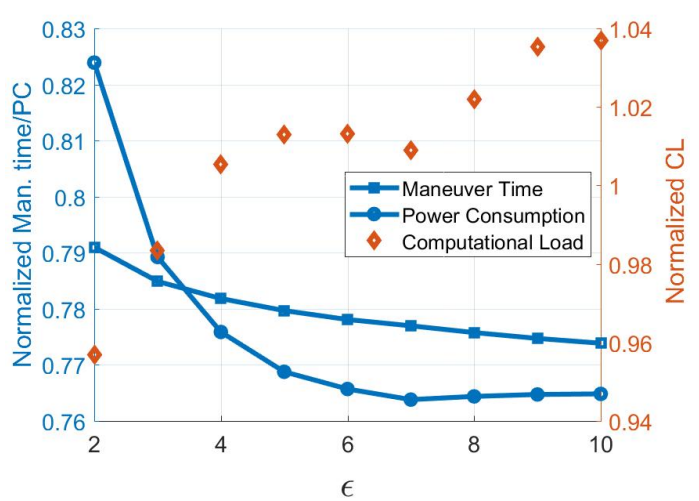

Figure 6.7: Normalized Man. time, $\mathrm{PC}$ and CL.

Figure 6.7 shows the normalized maneuver time (Man. time), the normalized power consumption (PC) (on the left axis,) and the normalized computational load (CL) (on the right axis,) for the proposed algorithm, for different values of $\epsilon$. The normalized PC and maneuver time are always less than 1, which means less power consumption and less maneuver time compared to the reference algorithm, for all $\epsilon$. Both the $\mathrm{PC}$ and the maneuver time improve (decrease) as $\epsilon$ increases, up to a point. As $\epsilon$ increases beyond the value of 6 , the change in $\bar{\delta}$ becomes very small. For example, the proposed duty cycle is $\bar{\delta}=0.95$ at $\epsilon=6$, while at $\epsilon=10$, it is $\bar{\delta}=0.97$, see Eq. (5.1). Increasing $\epsilon$, however, increases the computational load as shown on the right vertical axis in fig. 6.7 .

Figure 6.8 shows the change of the $\mathrm{CC}$ with $\epsilon$ on the left axis. On the right axis, the NRMSE and SI are depicted. The CC is close to 1, and the SI and NRMSE are very small, indicating strong correlation between the estimated and the true magnetic fields. Figure 6.9 shows the bias, RMSE, and MAE. The variation of all parameters 


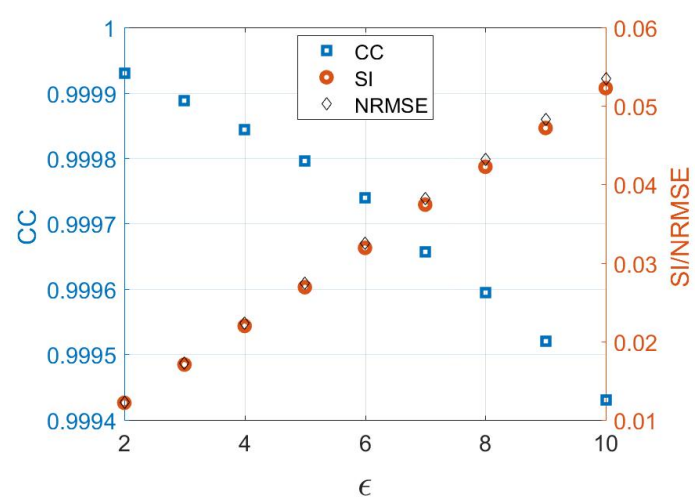

Figure 6.8: CC, SI and NRMSE of the $\mathbf{B}_{\text {sdo }}$ vector.

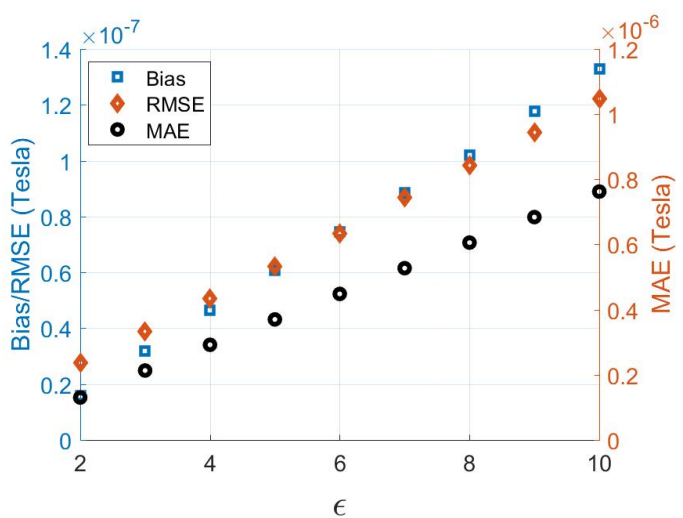

Figure 6.9: Bias, MAE and RMSE of the $\mathbf{B}_{\text {sdo }}$ vector.

confirms that the lower the $\epsilon$ the better ambient magnetic field estimation. In the following Monte Carlo analysis, the $\epsilon$ value is selected to be 6 which means $\bar{\delta}=0.95$ compared to $\delta=0.7$ in the reference algorithm.

\section{Monte Carlo simulation analysis}

Results are here presented for 11,000 Monte Carlo runs; 5,500 for the reference algorithm and 5,500 for the proposed algorithm. The 5,500 runs is categorized into 550 different categories. Each category has different initial angular rates (true and estimated), initial quaternion (true and estimated), bias vectors, and desired attitude. These values are generated randomly, and are the same for both algorithms. Table 6.1 shows the estimate of the magnitude of the disturbance torques, in the worst-case of the entire MC runs. 
Table 6.1

Worst-case disturbance torque magnitudes

\begin{tabular}{lc}
\hline \hline Disturbance & Magnitude $[N . m]$ \\
\hline Aerodynamic drag & $5.1910^{-9}$ \\
Gravity gradient & $1.0410^{-8}$ \\
Residual dipole & $4.0610^{-9}$ \\
Solar radiation & $1.0710^{-9}$ \\
\hline
\end{tabular}

Figure 6.10 depicts the reduction percentage in maneuver time for the 550 categories. Figure 6.10 shows that the maneuver time of the proposed algorithm is significantly less than that of the reference case. In some cases, the proposed algorithm achieves a reduction in the maneuver time of about $80 \%$, whereas in other cases there is almost no improvement in terms of the maneuver time, compared to the reference algorithm. The mean value of the savings in terms of the maneuver time is $31.4 \%$, with $19.89 \%$ standard deviation. In terms of the power consumption, the proposed algorithm reduces the normalized mean of the power consumption significantly compared to the reference algorithm as shown in fig 6.11 . The savings in terms of the power consumption, using the proposed algorithm, goes up to about $57 \%$ in some cases. The mean value for the power savings using the proposed algorithm is $24.97 \%$, with $11.63 \%$ standard deviation. The computational load is computed for the entire maneuver. Overall, the proposed algorithm has higher computational load compared to the reference algorithm because of the additional computations in evaluating $\mathbf{B}_{\text {sdo }}$ and in the MEKF magnetic propagation step. It is noticed that the computational load of the proposed algorithm roughly needs about $32 \%$ more computational resources at every time step. However, when the savings in the maneuver time is significant, the 


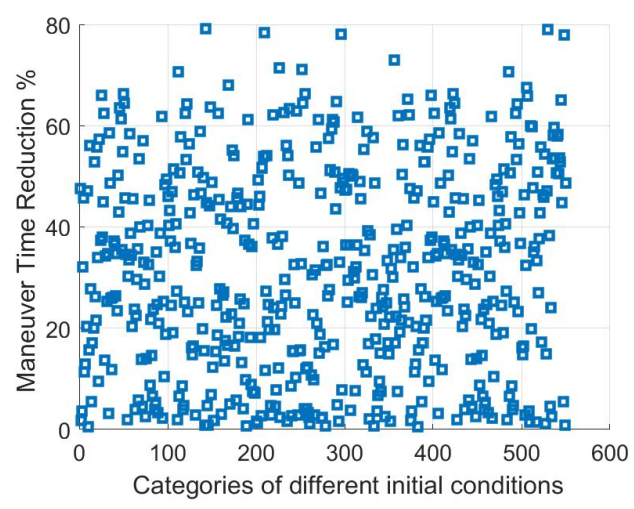

Figure 6.10: Maneuver time reduction percentage.

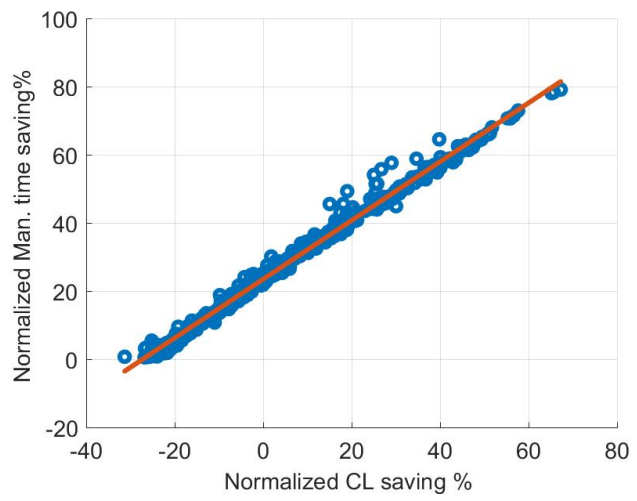

Figure 6.12: Normalized computational load versus normalized maneuver time.

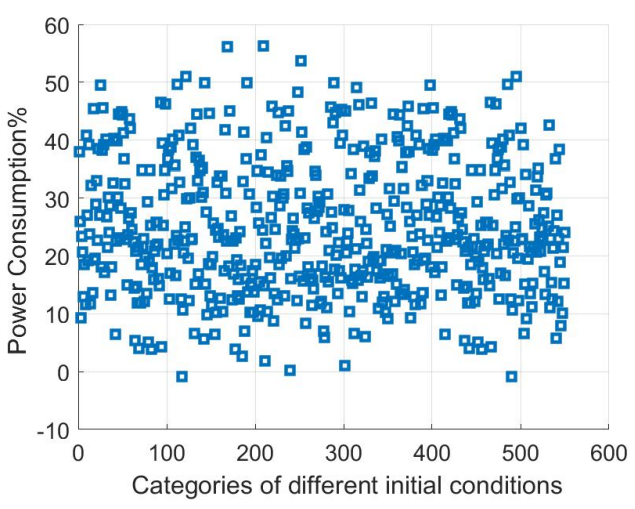

Figure 6.11: Power consumption reduction percentage.

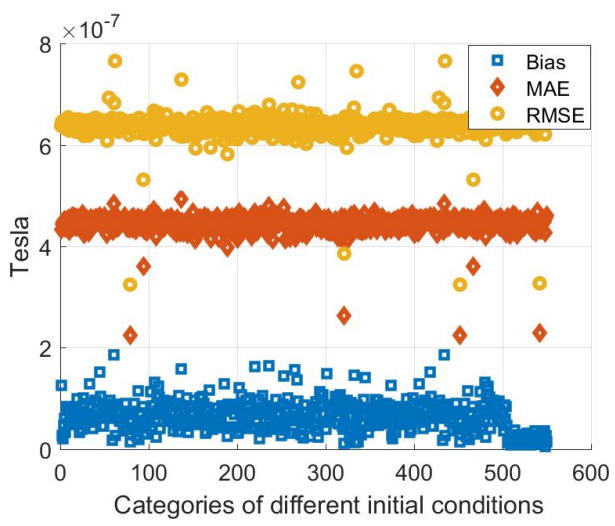

Figure 6.13: The mean of the RMSE, MAE and Bias at $\epsilon=6$.

computational time of the proposed algorithm becomes less than that of the reference algorithm, simply because the whole maneuver is complete in a significantly shorter period of time, and hence the computations stop much sooner compared to the reference algorithm. This observation is evident in fig 6.12 , where there is a strong correlation between the savings in the maneuver time and the computational load savings. 


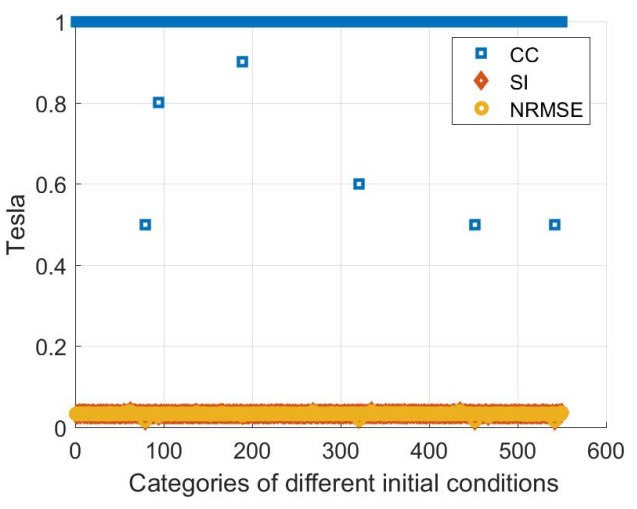

Figure 6.14: The mean of the CC, SI and NRMSE at $\epsilon=6$.

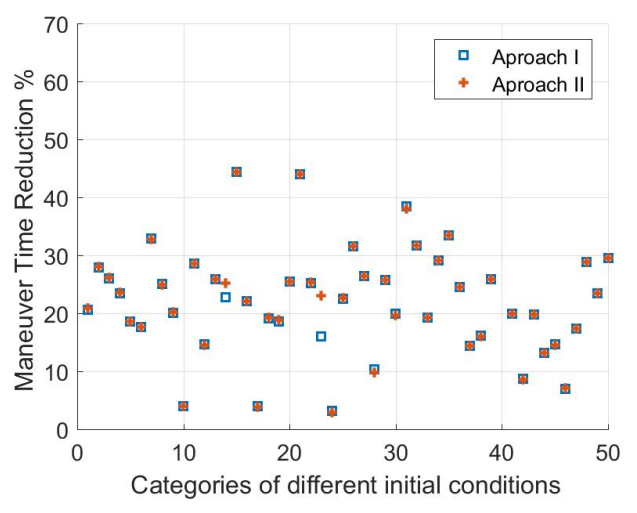

Figure 6.15: Normalized maneuver time for the proposed algorithm, $\epsilon=10$

Table 6.2

Mean and standard deviation of the six parameters

\begin{tabular}{|c|c|c|}
\hline Parameters & Mean & Standard deviation \\
\hline RMSE & $6.37010^{-7}[$ Tesla $]$ & $3.16710^{-8}[$ Tesla $]$ \\
\hline Bias & $6.37610^{-8}[$ Tesla $]$ & $2.97910^{-8}[$ Tesla $]$ \\
\hline MAE & $4.4311^{-7}[$ Tesla $]$ & $2.18510^{-8}[$ Tesla $]$ \\
\hline NRMSE & 0.0326 & 0.001 \\
\hline SI & 0.0323 & 0.001 \\
\hline $\mathrm{CC}$ & 0.9953 & 0.042 \\
\hline
\end{tabular}

Finally, regarding the estimated magnetic field parameters in the above MC simulations, all six parameters are computed as follows. Figure. 6.13 shows the computed average values of the RMSE, Bias and MAE in all categories. As can be seen in fig. 6.13, the average values indicate good estimate of the magnetic field. Figure. 6.14 shows the computed average values of the CC, SI and NRMSE. A strong correlation and less scattering is also observed in these MC runs. The mean and standard deviation values for the six parameters are presented in table 6.2 . 


\section{Magnetic Field Pseudo Measurement Methods Comparison}

In this section, a compression is considered between the methods for computing the magnetic field pseudo measurement to be used with the proposed ADCS. The method that solves the mathematical singularity from geometric point of view, see section 6.1.1. will be labeled (Approach I). The method that solves the mathematical singularity using the Tikhonov regularization approach, see section 5.2, will be labeled (Approach II). A 50 different regulation maneuver cases are performed with $\epsilon=10$. Where each case has different initial conditions and different noise seeds for the sensor's measurements. The maneuver time reduction, magnetic rods power saving and the total required computational resources for both approaches normalized with the results from the reference case are presented for the 50 cases in Figs. 6.15, 6.16 and 6.17 respectively. As can been seen from Figs. 6.15 and 6.16 that both approaches have almost the same saving in maneuver time and magnetic rods power consumption. However, approach II has the less required computational load. That was expected as approach $I$ needs to compute the Eigenvalues and Eigenvectors for the $[M]_{x}$. Moreover, an in-line 1-D optimization process is required to find the optimal regularization parameter $\alpha$ for the L-curve criteria [62]. 


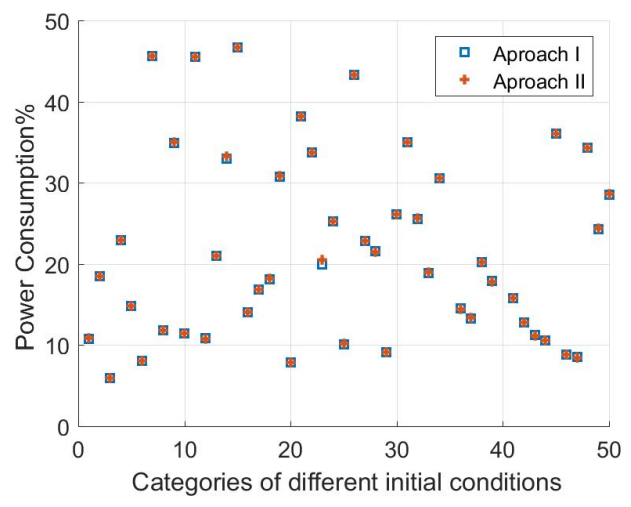

Figure 6.16: Normalized power consumption for the proposed algorithm, $\epsilon=10$

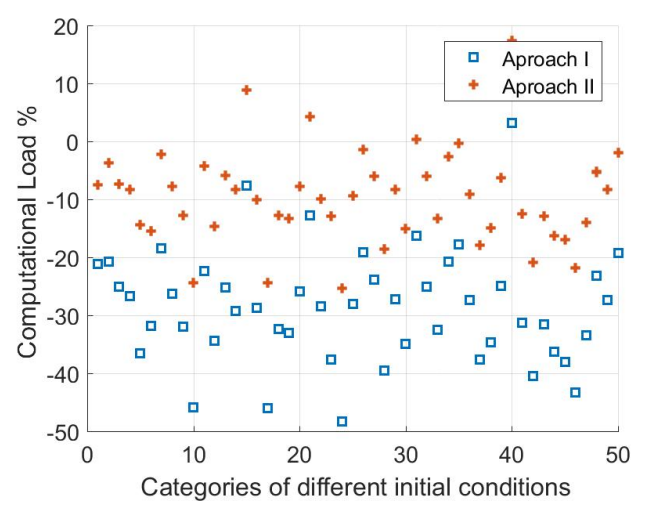

Figure 6.17: Normalized computational load for the proposed algorithm, $\epsilon=10$

\subsection{Verification of magnetic field Pseudo Measure- ment Computation using real data}

This section is dedicated to verify the magnetic field pseudo measurement computation process that is presented in section 6.1.1. The verification process will be conducted using real telemetry data from the CASSIOPE spacecraft. A brief overview over the CASSIOPE spacecraft hardware configuration, mission and the available telemetry data with its frequency is given in section 2.5. Furthermore, the procedures for estimating the angular velocity bias and inertia tensor elements are discussed in details in section 5.5.

Here, another $\mathrm{EKF}_{B}$ is used for magnetic field estimation, where the pseudo measurement $\mathbf{B}_{\text {sdo }}$ is the $\mathrm{EKF}_{B}$ input measurement. The magnetic field propagation model 
Eq. 6.14 propagates the magnetic field using the attitude parameters obtained from the star tracker. The following three diagonal covariance matrices are used: initial state covariance $[P]=\operatorname{diag}\left(\begin{array}{lll}1 & 1 & 1\end{array}\right) * 2 e^{-6}$, measurement noise error covariance $[R]=\operatorname{diag}\left(\begin{array}{lll}1 & 1 & 1\end{array}\right) * 0.5 e^{-4}$, and process noise error covariance $[Q]=\operatorname{diag}\left(\begin{array}{lll}1 & 1 & 1\end{array}\right) * 0.5 e^{-8}$. The magnetic field is filtered and estimated for the real data case where the system model equation is Eq. (6.14) and the measurement are the pseudo measurements, see sections 6.1.1 and 5.2, or the true ones. The Jacobean matrix that will be used for computing the state transition matrix $[\phi]$ is as follows:

$$
[F(x)]=\left[R\left(\mathbf{q}_{k \mid k-1}\right)\right]\left[R\left(\mathbf{q}_{k-1 \mid k-1}^{-1}\right)\right]
$$

The linearized form of the measurements matrix is as in Eq. (5.31). It is worth noting that the two EKFs can be combined together (the one that estimates the angular velocity $\left(\mathrm{EKF}_{\omega}\right)$ and the one that estimates magnetic field $\left.\left(\mathrm{EKF}_{B}\right)\right)$. However, they are implemented separately in this study.

For the first $200 \mathrm{sec}$ maneuver, using $\epsilon=5$, fig. 6.18 shows the un-biased angular velocity measurement versus the estimated one using the EKF. The good match proves that the rigid body dynamic model is able to reasonably represent the CASSIOPE spacecraft dynamic motion. Figure 6.19 shows the comparison between the $\mathrm{x}$ components of each of the real magnetic field measurement (labeled "True"), the pseudo 

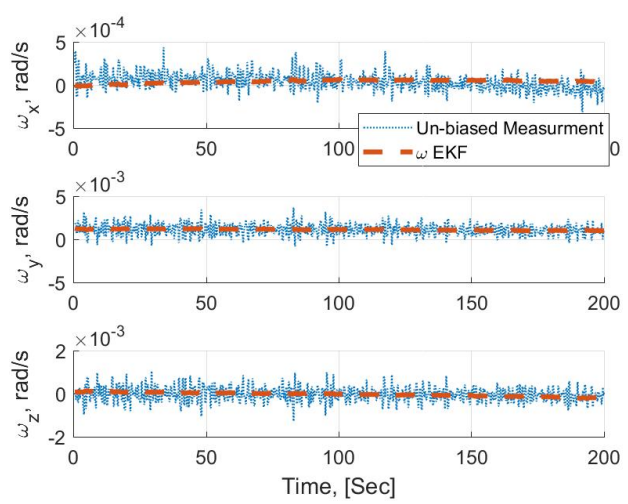

Figure 6.18: Angular velocities history from unbiased gyroscope measurements and the EKF output for $1^{\text {st }}$ maneuver.

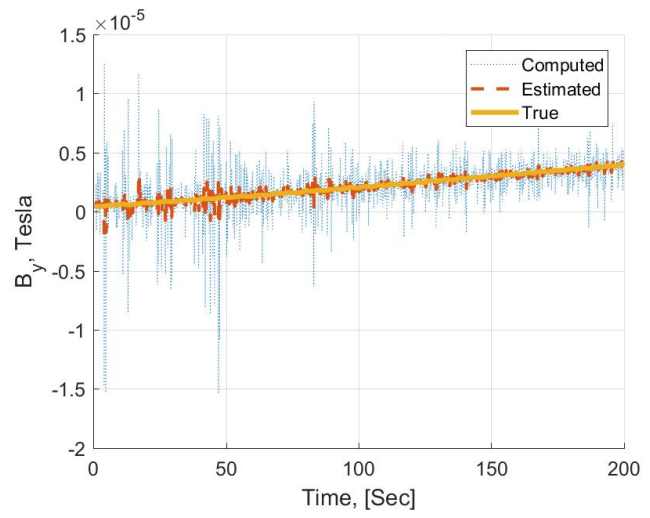

Figure 6.20: Magnetic field history in the $\mathrm{Y}$ direction for $1^{\text {st }}$ maneuver.

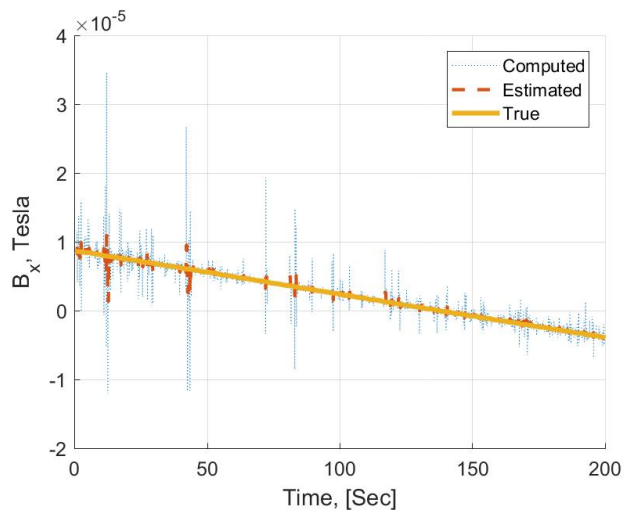

Figure 6.19: Magnetic field history in the $\mathrm{X}$ direction for $1^{\text {st }}$ maneuver.

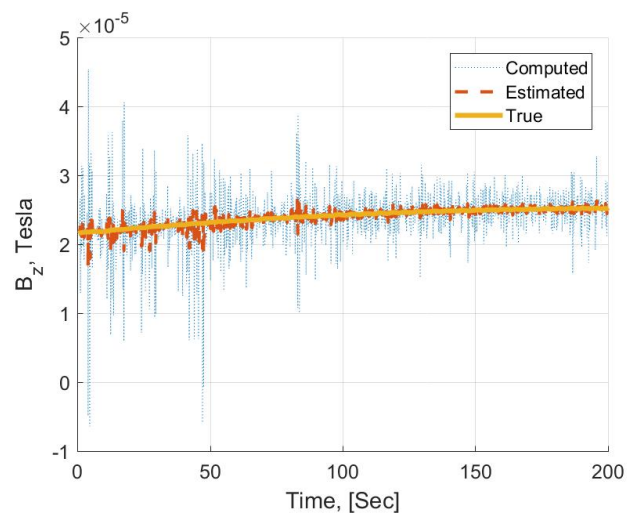

Figure 6.21: Magnetic field history in the $\mathrm{Z}$ direction for $1^{\text {st }}$ maneuver.

measurement $\mathbf{B}_{\text {sdo }}$ (labeled "Computed",) and the EKF estimated values (Labeled "Estimated").

Figures 6.19, 6.20 and 6.21 render the good performance of the estimation process in the $\mathrm{x}, \mathrm{y}$ and $\mathrm{z}$ directions, respectively. The six-validation parameters are computed for different values of $\epsilon$ and the results are plotted in figs 6.22 and 6.23 . The results here are in agreement with the conclusions from the Monte Carlo analysis; the lower 


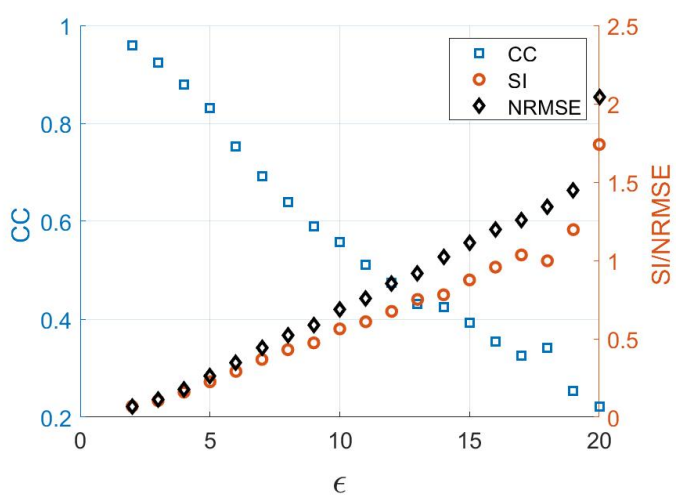

Figure 6.22: CC, SI and NRMSE of the estimated magnetic field for $1^{\text {st }}$ maneuver.

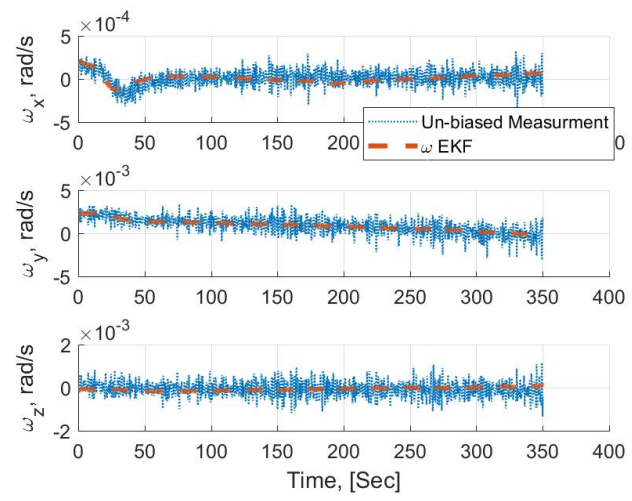

Figure 6.24: Angular velocities history from unbiased gyroscope measurements and the EKF output for $2^{\text {nd }}$ maneuver.

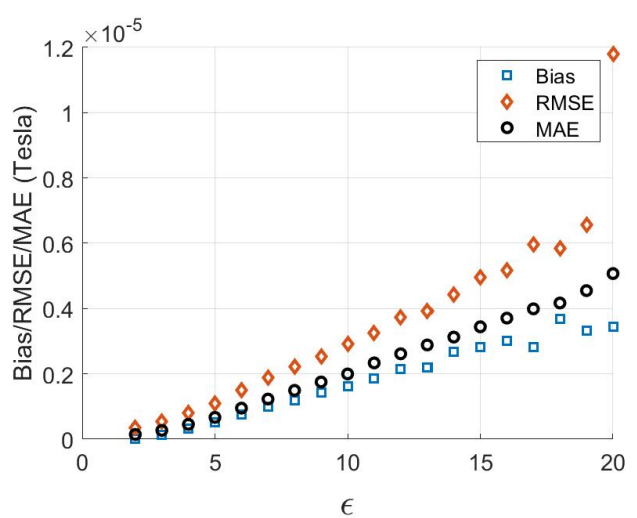

Figure 6.23: Bias, MAE and RMSE of the estimated magnetic field for $1^{\text {st }}$ maneuver.

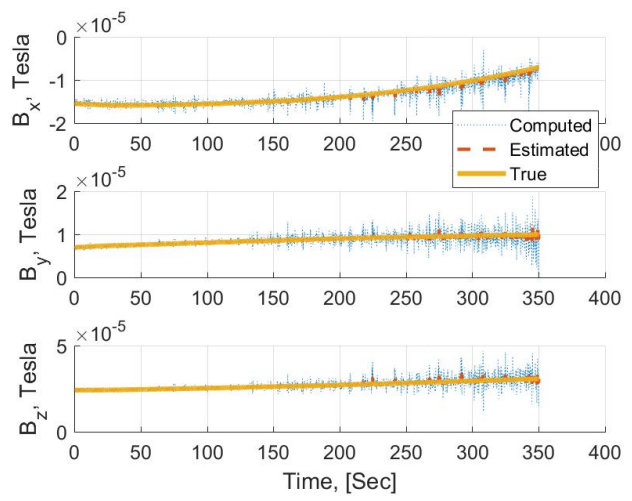

Figure 6.25: Magnetic field histories for the $2^{\text {nd }}$ maneuver.

the $\epsilon$ the better the magnetic field estimation accuracy.

Another maneuver of $300 \mathrm{~s}$ duration is also verified. The angular velocities histories are shown in fig.6.24. The magnetic field estimation values compared with the pseudo measurements and the true measurement are plotted in fig. 6.25. The magnetic field estimation accuracy is good, as evident from fig. 6.25. This is also confirmed by the values of the six validation parameters, which are listed in table 6.3, for this maneuver, 


\section{Table 6.3}

Validation parameters for the second maneuvers

\begin{tabular}{cccccc}
\hline CC & SI & NRMSE & RMSE [Tesla] & MAE [Tesla] & Bias [Tesla] \\
\hline 0.997 & 0.021 & 0.022 & $3.357 e^{-7}$ & $4.852 e^{-8}$ & $1.844 e^{-7}$ \\
\hline
\end{tabular}

using $\epsilon=5$.

It can be concluded that, the magnetic field estimation process is further refined by using the proposed ADCS scheme compared to the one that only computing the magnetic field pseudo measurement. This better estimate accompanied by lower computational demands if the magnetic field pseudo measurement process is carried out by the approach in section 6.1.1. Also it is observed that both approaches in section 6.1 .1 and 5.2 almost have the same performance in terms of power consumption and maneuver time.

\subsection{Conclusion}

An estimation algorithm was presented for spacecraft attitude that enables more efficient operation of the magnetic rods in attitude maneuvers. The proposed algorithm estimates the spacecraft attitude, in addition to the magnetic field, at the times when the magnetometer is not used. The magnetic field estimation process using the proposed MEKF gives better results compared to the approaches that compute the magnetic field pseudo measured only. The attitude error is within an acceptable 
accuracy for small spacecraft. An improvement in terms of the maneuver time and power consumption is fulfilled with acceptable computational demands . The magnetic field estimation process was tested against real data from CASSIOPE mission, and demonstrated good estimation accuracy. 


\section{Chapter 7}

\section{Conclusion and Future Work}

This chapter starts by the conclusion of the proposed work. Then the possible extension work as a future topics

\subsection{Conclusion}

1. For the detumbling maneuver, a novel variant of the B-dot law (TOC B-dot law) is developed. A substitute of spacecraft angular velocity is extracted from the derivative of the measured ambient magnetic field information. This substitute

of spacecraft angular velocity is used for computing the required torque to satisfy the TOC without measuring the angular velocity. Most work in the literature, 
uses the derivative of the measured ambient magnetic field as an indication of the spacecraft angular velocity. An improvement in detumbling time, power consumption and system stability is achieved using the proposed TOC B-dot law.

2. Another variant of the B-dot law (PMP B-dot law) is developed in the context of the optimal control theory. This control is derived from the first order optimality necessary conditions using the Pontryagin Minimum Principal, where the control effort is constrained. It has been shown that the Triple Orthogonality Condition (TOC), among the spacecraft's applied torque, the generated dipole moment and the ambient magnetic field vectors are the most effective solution. Furthermore, this law uses the extracted spacecraft angular velocity from the derivative of the measured ambient magnetic field as the case with the TOC B-dot law. The results show that the performance of both laws, TOC B-dot law, and PMP B-dot law, is very close.

3. Most existing B-dot laws in the literature neglect a residual term, which leads to a conclusion that these B-dot laws are asymptotically stable. This work has demonstrated that these B-dot laws are actually only Langrange stable when the residual term is taken into consideration.

4. An angular velocity-based detumbling control law is derived from the first order necessary conditions of optimality using the Pontryagin Minimum Principal. The performance is demonstrated to achieve detumbling in less maneuver time 
compared to most existing similar algorithms. This is confirmed through analysis and numerical simulations.

5. Considering intermittent activation of the magnetic rods for the proportional derivative control law for three-axis attitude maneuver, it is shown that the system is stable in the sense of Lagrange stability. There will be steady state error that is function of the disturbance torque, duty cycle and proportional gain. The steady state error is inversely proportional to the average duty cycle. Therefore, increasing the magnetic rods duty cycle reduces the steady state error.

6. A novel efficient control scheme that enables the magnetic rods longer operation is developed, by replacing the need for real magnetic field measurement with magnetic field pseudo measurement. The principal of the proposed scheme is to measure the rotational motion of the spacecraft when applying known magnetic dipole moment command. A Tikhonov regularization method is used to extract the magnetic field pseudo measurement information in this singular problem. The results confirm the less maneuver time with less power consumption achieved; yet high computational demands are required. furthermore, a validation cases are conducted using the CASSIOPE real telemetry data.

7. A complete attitude determination and control scheme is further developed that estimates the controller required information (angular velocity, attitude information, and magnetic field). This control scheme is implemented through 
Multiplicative Extended Kalman Filter (MEKF) that uses a relatively simple and fast dynamic model for the magnetic field along with dynamic and kinematic models. The results and the real data validation cases from CASSIOPE mission show that the estimation process using MEKF is effective in estimating the magnetic field and the spacecraft states.

\subsection{Future Work}

In this section, several possible extension of the work presented in this dissertation are outlined.

1. The problem of minimum-time spacecraft three-axis attitude maneuvers using magnetic rods shall be studied in the context of optimal control theory. It is recommended to treat the magnetic field as a function of time and spacecraft attitude, where the evolution of the attitude depends on the angular velocity, which depends on the control. Therefore, it seems a full treatment of the problem would need to include the attitude in the state vector, and correspondingly expand the co-state vector. This future work was pointed out by an anonymous reviewer of one of the publications produced from this dissertation.

2. The effect of the intermittent operation of the magnetic rods in magnetic three axis attitude control using PID controller; especially from the stability and 
steady state error perspectives.

3. Recently, the RemoveDEBRIS satellite in a recent successful experiment in September 2018 has demonstrated a new technology to catch a space debris from Surrey Space Center at Surrey University in UK, along with other several partners. The experiment successfully captured a deployed target CubeSat (that mimics a space debris), from mother spacecraft, by launching a net. Then the small spacecraft, CubeSat, deployed a large drag sail to increase the CubeSat deceleration and return to the earth atmosphere ${ }^{1}[108,109]$. Due to the large number of space debris, such a technology will require many small spacecraft, CubeSats. The lifetimes of these CubeSats are very short; and only a coarse attitude control system is needed. Therefore, low cost-effective solutions are highly desirable. The proposed control schemes that used for extending the magnetic rods activation time, may be used on such CubeSats for that purpose, by extending the number of combined cycles to infinity. In other words, removing the magnetometer for this limited time missions. Or using the proposed work in case of magnetometer malfunction.

${ }_{1}^{1}$ https://www.surrey.ac.uk/news/net-successfully-snares-space-debris 



\section{References}

[1] Avanzini, G. and Giulietti, F., "Magnetic Detumbling of a Rigid Spacecraft," Guidance, Control, and Dynamics, Vol. 35, No. 4, 2012, pp. 1326 - 1334.

[2] Silani, E. and Lovera, M., "Magnetic spacecraft attitude control: a survey and some new results," Control Engineering Practice, Vol. 13, No. 3, 2004, pp. 357371.

[3] Wang, P., Shtessel, Y. B., and Wang, Y. Q., "Satellite attitude control using only magnetorquers," 30th Southeastern Symposium on System Theory, Vol. 1, 1998, pp. 500-504.

[4] Lovera, M., "Magnetic Satellite Detumbling: The B-dot Algorithm Revisited," IEEE, American Control Conference (ACC), Vol. 146, 2015, pp. 66-72.

[5] Bouwmeester, J. and J.Guo, "Survey of Worldwide Pico-and Nanosatellite Missions, Distributions and Subsystem Technology," Acta Astronautica, Vol. 67, 2010, pp. 854-862. 
[6] Ivanov, D. S., Ovchinnikov, M. Y., Penkov, V. I., Roldugin, D. S., Doronin, D. M., and Ovchinnikov, A. V., "Advanced Numerical Study of the threeaxis Magnetic Attitude Control and Determination with Uncertainties," Acta Astronautica, Vol. 132, No. 3, 2017, pp. 103 - 110.

[7] Lovera, M. and Astolfi, A., "Spacecraft attitude control using magnetic actuators," Automatica, Vol. 40, No. 8, 2004, pp. 1405 - 1414.

[8] Wertz, J. R., Spacecraft attitude determination and control, Springer, 1978.

[9] Sidi, M. J., Spacecraft dynamics and control, Cambridge University Press, Cambridge, England, 1997.

[10] Astolfi, A. and Lovera, M., "Global spacecraft attitude control using magnetic actuators," American Control Conference, Vol. 2 of Proceedings of the 2002, IEEE, Anchorage, AK, USA, 2002, pp. 1331-1335.

[11] Lovera, M. and Astolfi, A., "Global Magnetic Attitude Control of Inertially Pointing Spacecraft," Journal of Guidance, Control, and Dynamics, Vol. 28, No. 5, 2005, pp. $1065-1072$.

[12] Sugimura, N., Kuwahara, T., and Yoshida, K., "Attitude Determination and Control System for Nadir Pointing Using Magnetorquer and Magnetometer," Aerospace Conference, 2016, pp. - . 
[13] Gerhardt, D. T. and Palo, S. E., "Passive Magnetic Attitude Control for CubeSat Spacecraft," Conference on Small Satellites, 24th Annual AIAA/USU, AIAA, Logan, UT,USA, 2010, pp. - .

[14] Humphreys, T. E., Psiaki, M. L., Klatt, E. M., Powell, S. P., and Kintner, P. M., "Magnetometer-based Attitude and Rate Estimation for a Spacecraft with Wire Booms," Journal of Guidance, Control, and Dynamics, Vol. 28, No. 4, 2005, pp. $584-593$.

[15] Siahpush, A. and Sexton, A., "A study for semi-passive gravity gradient stabilization of small satellites," Conference on Small Satellites, 1st Annual USU Conference on Small Satellites, AIAA, Logan, UT,USA, 1987, pp. - .

[16] Arduini, C. and Baiocco, P., "Active Magnetic Damping Attitude Control for Gravity Gradient Stabilized Spacecraft," Journal of Guidance, Control, and Dynamics, Vol. 20, No. 1, 1997, pp. 117- 122.

[17] Martel, F., Pal, P. K., and Psiaki, M. L., "Active Magnetic Control System for Gravity Gradient Stabilized Spacecraft," Conference on Small Satellites, 2nd Annual AIAA/USU Conference on Small Satellites, AIAA, Logan, UT, USA, 1988, pp. - .

[18] Stickler, A. C. and Alfriend, K. T., "Elementary Magnetic Attitude Control System," Journal of Spacecraft and Rockets, Vol. 13, No. 5, 1976, pp. 282- 287. 
[19] Alfriend, K. T., "Magnetic Attitude Control System for Dual-Spin Satellites," AIAA Journal, Vol. 13, No. 6, 1975, pp. 817-822.

[20] Vatankhahghadim, B. and Damaren, C. J., "Magnetic Attitude Control with Impulsive Thrusting Using the Hybrid Passivity Theorem," Journal of Guidance, Control, and Dynamics, Vol. 40, No. 8, 2017, pp. 1860-1876.

[21] Vatankhahghadim, B. and Damaren, C. J., "Optimal Combination of Magnetic Attitude Control with Impulsive Thrusting," Journal of Guidance, Control, and Dynamics, Vol. 39, No. 10, 2016, pp. 2391- 2398.

[22] de Angelis, E. L., Giulietti, F., de Ruiter, A. H. J., and Avanzini, G., "Spacecraft Attitude Control Using Magnetic and Mechanical Actuation," Journal of Guidance, Control, and Dynamics, Vol. 39, No. 3, 2016, pp. 564- 573.

[23] Forbes, J. R. and Damaren, C. J., "Geometric Approach to Spacecraft Attitude Control Using Magnetic and Mechanical Actuation," Journal of Guidance, Control, and Dynamics, Vol. 33, No. 2, 2010, pp. 590- 595.

[24] Celani, F., "Spacecraft Attitude Stabilization Using Magnetorquers with Separation Between Measurement and Actuation," Journal of Guidance, Control, and Dynamics, Vol. 39, No. 9, 2016, pp. 2180-2187.

[25] Lovera, M., "Spacecraft attitude and rate estimation from vector observations: a comparison study," European Control Conference (ECC), IEEE, Porto, Portugal, 2001, pp. - . 
[26] Stickler, A. C. and Alfriend, K., "Elementary Magnetic Attitude Control System," AIAA Spacecraft and Rockets, Vol. 13, No. 5, 1976, pp. 282-287.

[27] Fonod, R. and Gill, E., "Magnetic Detumbling of Fast-tumbling Picosatellites," $69^{\text {th }}$ International Astronautical Congress, International Astronautical Federation, IAF, 2018, pp. 1-11.

[28] Zavoli, A., Giulietti, F., Avanzini, G., and Matteis, G. D., "Spacecraft Dynamics Under the Action of Y-dot Magnetic Control Law," Acta Astronautica, Vol. 122, 2016, pp. 146-158.

[29] Cubas, J., Farrahi, A., and Pindado, S., "Magnetic Attitude Control for Satellites in Polar or Sun-Synchronous Orbits," Journal of Guidance, Control, and Dynamics, Vol. 38, No. 10, 2015, pp. 1947-1958.

[30] Karpenko, S. O., Ovchinnikov, M. Y., Roldugin, D. S., and Tkachev, S. S., "One-axis Attitude of Arbitrary Satellite Using Magnetorquers Only," Cosmic Research, Vol. 51, No. 6, 2013, pp. 478-484.

[31] Carletta, S. and Teofilatto, P., "Design and Numerical Validation of an Algorithm for the Detumbling and Angular Rate Determination of a CubeSat Using Only Three-Axis Magnetometer Data," International Journal of Aerospace Engineering, Vol. 2018, No. 9768475, 2018, pp. 1-12. 
[32] Ovchinnikov, M. Y., Penkov, V. I., Roldugin, D. S., and Karpenko, S. O., "Investigation of the Effectiveness of an Algorithm of Active Magnetic Damping," Cosmic Research, Vol. 50, No. 2, 2012, pp. 170-176.

[33] Ovchinnikov, M. Y., Roldugin, D. S., Penkov, V. I., Varatarao, R., and Ryabikov, V. S., "Motion of a Satellite Equipped With a Pitch Flywheel and Magnetic Coils in Gravitational Field," Cosmic Research, Vol. 55, No. 3, 2017, pp. 207-213.

[34] Psiaki, M. L., "Magnetic Torquer Attitude Control via Asymptotic Periodic Linear Quadratic Regulation," Journal of Guidance, Control, and Dynamics, Vol. 24, No. 2, 2001, pp. 386-394.

[35] Pittelkau, M. E., "Optimal Periodic Control for Spacecraft Pointing and Attitude Determination," Journal of Guidance, Control, and Dynamics, Vol. 16, No. 6, 1993, pp. 1078-1084.

[36] Findlay, E. J., de Ruiter, A., Forbes, J. R., Liu, H., Damaren, C. J., and Lee, J., "Magnetic Attitude Control of a Flexible Satellite," Journal of Guidance, Control, and Dynamics, Vol. 36, No. 5, 2013, pp. 1522-1527.

[37] Lovera, M., Marchi, E. D., and Bittanti, S., "Periodic Attitude Control Techniques for Small Satellites with Magnetic Actuators," IEEE Transactions on Control Systems Technology, Vol. 10, No. 1, 2002. 
[38] de Marchi, E., de Rocco, L., Morea, G. D., and Lovera, M., "Optimal Magnetic Momentum Control for Inertially Pointing Spacecraft," Spacecraft Guidance, Navigation and Control Systems, Vol. 7, No. 1, 1999, pp. 30-39.

[39] Ovchinnikov, M. Y., Roldugin, D., Tkachev, S., and Penkov, V., "B-dot Algorithm Steady-State Motion Performance," Acta Astronautica, Vol. 146, 2018, pp. 66-72.

[40] Lovera, M. and Astolfi, A., "Global Magnetic Attitude Control of Inertially Pointing Spacecraft," Journal of Guidance, Control, and Dynamics, Vol. 28, No. 5, 2005, pp. 1065-1072.

[41] Wen, J. T. Y. and Kreutz-Delgado, K., "The attitude control problem," Automatic Control, Vol. 36, No. 10, 1991, pp. 584 - 593.

[42] Lovera, M. and Astolfi, A., "Global magnetic attitude control of spacecraft in the presence of gravity gradient," IEEE transaction on Aerospace and Electronic Systems, Vol. 42, No. 3, 2006.

[43] Bolandi, H. and Vaghei, B. G., "Stable Supervisory-Adaptive Controller for Spinning Satellite using Only Magnetorquers," IEEE transaction on Aerospace and Electronic Systems, Vol. 45, No. 1, 2009.

[44] Vazquez, A. R., Prats, M. M., and Zazzera, F. B., "Spacecraft magnetic attitude control using approximating sequence Riccati equations," IEEE transaction on Aerospace and Electronic Systems, Vol. 51, No. 4, 2015. 
[45] Yang, Y., "Controllability of spacecraft using only magnetic torques," IEEE transaction on Aerospace and Electronic Systems, Vol. 52, No. 2, 2016.

[46] Arduini, C. and Baiocco, P., "Active Magnetic Damping Attitude Control for Gravity Gradient Stabilized Spacecraft," Journal of Guidance, Control, and Dynamics, Vol. 20, No. 1, 1997, pp. 117- 122.

[47] Lovera, M. and Astolfi, A., "Spacecraft Attitude Control Using Magnetic Actuators," Automatica, Vol. 40, No. 8, 2004, pp. 1405-1414.

[48] Celani, F., "Robust three-axis attitude stabilization for inertial pointing spacecraft using magnetorquers," Acta Astronautica, Vol. 107, 2015, pp. 87-96.

[49] Wisniewski, R. and Blanke, M., "Fully magnetic attitude control for spacecraft subject to gravity gradient," Automatica, Vol. 35, No. 7, 1999, pp. 1201-1214.

[50] PSIAKI, M. L., MARTEL, F., and PAL, P. K., "Three-axis attitude determination via Kalman filtering of magnetometer data," Journal of Guidance, Control, and Dynamics, Vol. 13, No. 3, 1990, pp. 506- 514.

[51] Tortora, P., Oshman, Y., and Santono., F., "Spacecraft Angular Rate Estimation from Magnetometer Data Only Using an Analytic Predictor," Journal of Guidance, Control, and Dynamics, Vol. 27, No. 3, 2004, pp. 365-373.

[52] Abdelrahman, M. and Park, S. Y., "Sigma-Point Kalman Filtering for Spacecraft Attitude and Rate Estimation using Magnetometer Measurements," IEEE 
transaction on Aerospace and Electronic Systems, Vol. 47, No. 2, 2011, pp. 1401 $-1415$.

[53] Egziabher, D. G., Elkaim, G. H., Powell, J. D., and Parkinson, B. W., "Calibration of Strapdown Magnetometers in the Magnetic Field Domain and Actuation," ASCE Journal of Aerospace Engineering, Vol. 19, No. 9, 2006, pp. 192191.

[54] Foster, C. C. and Elkaim, G. H., "Extension of a two-step calibration methodology to include nonorthogonal sensor axes," Aerospace and Electronic Systems, Vol. 44, No. 3, 2008.

[55] Burton, R., Rock, S., Springmann, J., and Cutlerd, J., "Dual attitude and parameter estimation of passively magnetically stabilized nano satellites," Acta Astronautica, Vol. 94, No. 1, 2014, pp. 145-158.

[56] Burton, R., Rock, S., Springmann, J., and Cutlerd, J., "Online attitude determination of a passively magnetically stabilized spacecraft," Acta Astronautica, Vol. 133, 2017, pp. 269-281.

[57] Bhat, S., "Controllability of nonlinear time-varying systems: applications to spacecraft attitude control using magnetic actuation," IEEE Transactions on Automatic Control, Vol. 50, No. 11, 2005, pp. 1725-1735.

[58] Coverstone-Carroll, V., "Detumbling and Reorienting Underactuated Rigid 
Spacecraft," Journal of Guidance, Control, and Dynamics, Vol. 19, No. 3, 1996, pp. $708-710$.

[59] Springmann, J. C., "Satellite Attitude Determination with Low-Cost Sensors," PhD dissertation, Aerospace Engineering Department, University of Michigan, 2013, pp. - .

[60] Wallis, D. D., Miles, D. M., Narod, B. B., Bennest, J. R., Murphy, K. R., Mann, I. R., and Yau, A. W., "The CASSIOPE/e-POP Magnetic Field Instrument (MGF)," Space Science Reviews, Vol. 189, No. 1, 2015, pp. 27-39.

[61] Celani, F., "Spacecraft Attitude Stabilization with Piecewise-Constant Magnetic Dipole Moment," Guidance, Control, and Dynamics, Vol. 39, No. 5, 2016, pp. $1140-1146$.

[62] Desouky, M. A. A. and Abdelkhalik, O. O., "Improved Spacecraft Magnetic Attitude Maneuvering," AIAA Journal of spacecraft and rockets, Vol. accessed May 17, 2019, pp. 1-13.

[63] Celani, F., "Magnetic Spacecraft Control via Output Feedback with Separation between Measurement and Actuation," Aerotecnica Missili and Spazio, Vol. 95, No. 2, 2016, pp. 71-81.

[64] Livermore, P. W., Hollerbach, R., and Finlay, C. C., "An accelerating highlatitude jet in Earth's core," Nature Geoscience, Vol. 10, 2017, pp. 62-68. 
[65] Aubert, J., "Geomagnetic acceleration and rapid hydromagnetic wave dynamics in advanced numerical simulations of the geodynamo," Geophysical Journal International, Vol. 214, No. 1, 2018, pp. 531-547.

[66] Ovchinnikov, M. Y. and Roldugin, D., "A Survey on Active Magnetic Attitude Control Algorithms for Small Satellites," Progress in Aerospace Sciences, Vol. in press, 2019.

[67] Juchnikowski, G., Barcinski, T., and Lisowski, J., "Optimal Control Gain for Satellite Detumbling Using B-dot Algorithm," $2^{\text {nd }}$ CEAS Specialist Conference on Guidance, Navigation and Control, 2013, pp. 1158-1168.

[68] Juchnikowski, G., Barcinski, T., and Lisowski, J., "Detumbling of Highly NonSymmetrical Satellites with Use of B-dot Control Law," $18^{\text {th }}$ International Conference on Methods Models in Automation Robotics (MMAR), IEEE, Hoboken, NJ, USA, 2013, pp. 582-587.

[69] Christoph B"ohm, Moritz Merk, W. F. and Allg“ower, F., "Spacecraft Rate Damping with Predictive Control Using Magnetic Actuators Only," Proceedings of the International Workshop on Assessment and Future Directions of Nonlinear Model Predictive Control, Vol. 384, Springer, Berlin, Heidelberg, 2009, pp. $511-520$.

[70] Ahmed, S. and Kerrigan, E. C., "Suboptimal Predictive Control for Satellite 
Detumbling," Journal of Guidance, Control, and Dynamics, Vol. 37, No. 3, 2014, pp. 850-859.

[71] Liu, H. Y., Wang, H. N., and Chen, Z. M., "Detumbling Controller and Attitude Acquisition for Micro-Satellite Based on Magnetic Torque," Journal of Astronautics, Vol. 28, No. 2, 2007, pp. 333-337.

[72] Yang, C.-C. and Wu, C.-J., "Time-Optimal Detumbling Control of a Rigid Spacecraft," Journal of Vibration and Control, Vol. 14, No. 4, 2008, pp. 553570.

[73] Romano, M., "Detumbling and Nutation Canceling Maneuvers with Complete Analytic Reduction for Axially Symmetric Spacecraft," Acta Astronautica, Vol. 66, No. 7-8, 2010, pp. 989-998.

[74] Aghili, F., "Time-Optimal Detumbling Control of Spacecraft," Journal of Guidance, Control, and Dynamics, Vol. 32, No. 5, 2009, pp. 1671-1675.

[75] Scrivener, S. L. and Thompson, R. C., "Survey of Time-Optimal Attitude Maneuvers," Journal of Guidance, Control, and Dynamics, Vol. 17, No. 2, 1994, pp. 225-233.

[76] Pulecchi, T., Lovera, M., and Varga, A., "Optimal Discrete-Time Design of Three-Axis Magnetic Attitude Control Laws," IEEE Transactions on Control Systems Technology, Vol. 18, No. 3, 2010. 
[77] Gravdahl, J. T., "Magnetic attitude control for satellites," 43rd IEEE Conference on Decision and Control, Atlantis, Paradise Island, Bahamas, 2004, pp.--.

[78] M.Yu.Ovchinnikov, D.S.Roldugin, and V.I.Penkov, "Three-Axis Active Magnetic Attitude Control Asymptotical Study," Acta Astronautica, Vol. 110, 2015, pp. $279-286$.

[79] M.Yu.Ovchinnikov, D.S.Roldugin, Ivanov, D., and V.I.Penkov, "Choosing Control Parameters for Three Axis Magnetic Stabilization in Orbital Frame," Acta Astronautica, Vol. 116, 2015, pp. 74-77.

[80] Abdelrahman, M. A. and young Park, S., "Integrated Attitude Determination and Control System via Magnetic Measurements and Actuation," Acta Astronautica, Vol. 69, No. 3-4, 2011, pp. 168-185.

[81] Cai, Guowei, C. B. M. and Lee, T. H., Unmanned Rotorcraft Systems, Springer, 2011.

[82] Schaub, H. and john L. Junkins, Analytical mechanics of space systems, AIAA Education Series, AIAA, New York, 2nd ed., 2003.

[83] Lay, D. C., Linear Algebra and Its Applications, Addison Wesley, Boston, USA, 3rd ed., 2006.

[84] Yau, A. W. and James, H. G., "CASSIOPE Enhanced Polar Outflow Probe 
(e-POP) Mission Overview," Space Science Reviews, Vol. 189, No. 1, Jun 2015, pp. 3-14.

[85] Khalil, H., Nonlinear Systems, Prentice-Hall, Englewood Cliffs, NJ, 3rd ed., 1996.

[86] Abramowitz, M., Handbook of Mathematical Functions, With Formulas, Graphs, and Mathematical Tables, NBS, 1964.

[87] Naidu, D. S., Optimal Control Systems, CRC Press, Inc., Boca Raton, FL, USA, 2002.

[88] Kirk, D. E., Optimal Control Theory: An Introduction, Dover Publications, USA, 2004.

[89] Desouky, M. A. A. and Abdelkhalik, O., "Improved Magnetic Attitude Control," NAECON (National Aerospace and Electronics Conference), IEEE, Hoboken, NJ, USA, 2019, pp. 1-8.

[90] Desouky, M. A. A. and Abdelkhalik, O., "Efficient Magnetic Attitude Regulation Control," AAS/AIAA Astrodynamics Specialist Conference Portland, ME, Vol. AAS-19-664, AAS, 2019, pp. 1-16.

[91] Hansen, P. C., Computational Inverse Problems in Electrocardiology, WIT Press, 2001. 
[92] Watkins, D. S., Fundamentals of Matrix Computations, John Wiley \& Sons, Inc., New York, NY, USA, 3rd ed., 2002.

[93] Doicu, A., Trautmann, T., and Schreier, F., Tikhonov regularization for linear problems, Springer Praxis Books. Springer, Berlin, Heidelberg, 2010.

[94] Kohavi, R., "A Study of Cross-Validation and Bootstrap for Accuracy Estimation and Model Selection," -, Morgan Kaufmann, 1995, pp. 1137-1143.

[95] Calvetti, D., Morigi, S., Reichel, L., and Sgallari, F., "Tikhonov regularization and the L-curve for large discrete ill-posed problems," Computational and Applied Mathematics, Vol. 123, No. 1-2, 2000, pp. 423-446.

[96] Giri, D., Mukherjee, B., N, B. T., and Sinha, M., "Three-Axis Global Magnetic Attitude Control of Earth-Pointing Satellites in Circular Orbit," Asian Journal of Control, Vol. 19, No. 6, 2017.

[97] Damaren, C. J., "Hybrid magnetic attitude control gain selection," Journal of Aerospace Engineering, Vol. 223, No. 8, 2009, pp. 1041-1047.

[98] Liang, J., Fullmer, R., and Chen, Y. Q., "Time-optimal magnetic attitude control for small spacecraft," $43^{\text {rd }}$ Conference on Decision and Control, Vol. 1, IEEE, Hoboken, NJ, USA, 2004, pp. 255-260.

[99] Bruni, R. and Celani, F., "A Robust Optimization Approach for Magnetic 
Spacecraft Attitude Stabilization," Journal of Optimization Theory and Applications, Vol. 173, No. 3, 2017, pp. 994-1012.

[100] M.Yu., O., D.S., R., D.S., I., and V.I., P., "Choosing control parameters for three axis magnetic stabilization in orbital frame," Acta Astronautica, Vol. 116, 2015, pp. $74-77$.

[101] M.Yu., O., D.S., R., and V.I., P., "Three-axis active magnetic attitude control asymptotical study," Acta Astronautica, Vol. 110, 2015, pp. 279 - 286.

[102] Giri, D. K. and Sinha, M., "Robust Backstepping Magnetic Attitude Control of Satellite Subject to Unsymmetrical Mass Properties," AIAA Journal of spacecraft and rockets, Vol. 1, 2019, pp. 298-305.

[103] Vallado, Fundamentals of Astrodynamics and Applications, Microcosm Press, Portland, OR, 4th ed., 2013.

[104] Crassidis, J. L. and john L. Junkins, Optimal Estimation of Dynamic Systems, Chapman and Hall/CRC Applied Mathematics and Nonlinear Science Series, CRC Press, FL, USA, 2nd ed., 2012.

[105] Unhelkar, V. and Hablani, H., "Spacecraft Attitude Determination with Sun Sensors, Horizon Sensors and Gyros: Comparison of Steady-State Kalman Filter and Extended Kalman Filter," Advances in Estimation, Navigation, and Spacecraft Control, ENCS 2012, Springer, Berlin, Heidelberg, 2015, pp. 413437. 
[106] Franklin, G. F., Powell, J. D., and Naeini, A. E., Feedback Control of dynamic System, Pearson, Upper Saddle River, NJ, 6th ed., 2010.

[107] Jazwinski, A. H., Stochastic Processes and Filtering Theory, Courier Corporation, 2015.

[108] L.Forshaw, J., S.Aglietti, G., Navarathinam, N., Kadhem, H., Salmon, T., Pisseloup, A., Joffre, E., Chabot, T., Retat, I., Axthelm, R., Barraclough, S., Ratcliffe, A., Bernal, C., Chaumette, F., Pollini, A., and H.Steynk, W., "RemoveDEBRIS: An in-orbit active debris removal demonstration mission," Acta Astronautica, Vol. 127, 2016, pp. 448-463.

[109] L.Forshaw, J., S.Aglietti, G., Salmon, T., Retat, I., Roed, M., Burgess, C., Chabot, T., Pisseloup, A., Phipps, A., Bernal, C., Chaumette, F., Pollini, A., and H.Steynk, W., "Final payload test results for the RemoveDebris active debris removal mission," Acta Astronautica, Vol. 138, 2017, pp. 326 - 342.

[110] Wertz, J. R., Spacecraft attitude determination and control, Springer, 1978.

[111] Pope, C. T., "Calibration and uncertainty analysis of a spacecraft attitude determination test stand," MSc degree project, Department of Computer Science, Electrical and Space Engineering, Luleå University of Technology, 2017, pp. -.

[112] Habib., T. M. A., "A comparative study of spacecraft attitude determination and estimation algorithms (a cost-benefit approach)," Aerospace Science and Technology, Vol. 26, No. 1, 2012, pp. 211-215. 
[113] k. Soh, W., Hamzah, N., and Arshad, A. S., "D-SAT simplified magnetic attitude control," Conference on Small Satellites, 18th Annual AIAA/USU, AIAA, Logan, UT,USA, 2004, pp. - .

[114] Desouky, M. A. A., Prabhu, K., and Abdelkhalik., O. O., "On Spacecraft Magnetic Attitude Control," 2018 Space Flight Mechanics Meeting, AIAA, 2018, pp. $1-11$.

[115] CHEN, R. M. D., "Asymptotic stability theorem for autonomous systems," Guidance, Control, and Dynamics, Vol. 16, No. 5, 1993, pp. 961-963.

[116] Bryson, A., Control of Spacecraft and Aircraft, Princeton University Press, New Jersey, 1994.

[117] Bak, T., Spacecraft Attitude Determination, Ph.D. thesis, Aalborg University, 1999.

[118] Segal, S., Carmi, A., and Gurfil, P., "Stereovision-Based Estimation of Relative Dynamics Between Noncooperative Satellites: Theory and Experiments," IEEE TRANSACTIONS ON CONTROL SYSTEMS TECHNOLOGY, Vol. 22, No. 2, 2014, pp. $568-584$.

[119] Shtark, T. and Gurfil, P., "Tracking a Non-Cooperative Target Using Real-Time Stereovision-Based Control: An Experimental Study," Sensors, Vol. 17, No. 4, 2017, pp. 1-29. 
[120] Reijneveld, J. and Choukroun, D., "Attitude Control System of The DelfiN3xt Satellite," Progress in Flight Dynamics, GNC, and Avionics, Vol. 6, 2013, pp. 189-208.

[121] Headrick, R. D.; Markley, F. L., "Onboard Magnetic Field Modeling for Solar Maximum Mission /SMM/," AIAA Conference, 1977.

[122] Thienel, J., Bruninga, R., Stevens, R., Ridge, C., and Healy, C., "The Magnetic Attitude Control System for the Parkinson Satellite (PSAT) A US Naval Academy Designed CubeSat," AIAA Guidance, Navigation, and Control Conference, Vol. AIAA 2009-5947, 2009, pp. 1-9.

[123] Miles, D. M., Mann, I. R., Ciurzynski, M., Barona, D., Narod, B. B., Bennest, J. R., Pakhotin, I. P., Kale, A., Bruner, B., Nokes, C. D. A., Cupido, C., Haluza-DeLay, T., Elliott, D. G., and Milling, D. K., "A miniature, low-power scientific fluxgate magnetometer: A stepping-stone to cube-satellite constellation missions," Journal of Geophysical Research: Space Physics, Vol. 121, No. 12, 2016, pp. 11,839-11,860.

[124] Azam, M., Singh, S., Iyer, A., and Kakad, Y., "Detumbling and Reorientation Maneuvers and Stabilization of NASA SCOLE System," IEEE transaction on Aerospace and Electronic Systems, Vol. 28, No. 1, 1992, pp. 80-91.

[125] Hegrenas, O., Gravdahl, J. T., and Tondel, P., "Attitude Control by Means of 
Explicit Model Predictive Control, via Multi-Parametric Quadratic Programming," American Control Conference, Vol. 2, IEEE, Hoboken, NJ, USA, 2005, pp. 901-906.

[126] Sharma, R. and Tewari, A., "Optimal Nonlinear Tracking of Spacecraft Attitude Maneuvers," IEEE Transactions on Control Systems Technology, Vol. 12, No. 5, 2004, pp. 677-682.

[127] Yang, Y., "Spacecraft Attitude Determination and Control: Quaternion Based Method," Annual Reviews in Control, Vol. 36, No. 2, 2012, pp. 198-219.

[128] Crassidis, J. L., Markley, F. L., and Cheng, Y., "Survey of Nonlinear Attitude Estimation Methods," Journal of guidance, control, and dynamics, Vol. 30, No. 1, 2007, pp. 12-48.

[129] Chessab, M. and Shehab, A., "Attitude Determination and Control System design of KufaSat," International Journal of Current Engineering and Technology, Vol. 4, No. 4, 2014, pp. 2910-2920.

[130] Aghili, F., "Time-Optimal Detumbling Control of Spacecraft," Journal of Guidance, Control, and Dynamics, Vol. 32, No. 5, 2009, pp. 1671-1675.

[131] Pulecchi, T., Lovera, M., , and Varga, A., "Optimal Discrete-Time Design of Three-Axis Magnetic Attitude Control Laws," IEEE Transactions on Control Systems Technology, Vol. 18, No. 3, 2010, pp. 714-722. 
[132] Fjellstad, O. E. and Fossen, T., "Comments on The attitude control problem," IEEE Transactions on Automatic Control, Vol. 39, No. 3, 1994, pp. 699 - 700.

[133] Desouky, M. A. A. and Abdelkhalik, O., "Efficient B-dot Law for Spacecraft Magnetic Detumbling," AAS/AIAA Astrodynamics Specialist Conference Portland, ME, Vol. AAS-19-665, AAS, 2019, pp. 1-17. 